\title{
Anomalous superconductivity and superfluidity in repulsive fermion systems
}

\author{
M. Yu. Kagan ${ }^{a, b}$, V.A. Mitskan ${ }^{c, d}$, M. M. Korovushkin ${ }^{c}$ \\ a) Kapitza Institute for Physical Problems, Russian Academy of Sciences, 119334 Moscow, Russian Federation \\ b) National Research University Higher School of Economics, 101000 Moscow, Russian Federation \\ ${ }^{c}$ Kirensky Institute of Physics, Siberian Branch of the Russian Academy of Sciences, 660036 Krasnoyarsk, Russian Federation \\ ${ }^{d)}$ Reshetnev Siberian State Aerospace University, 660014 Krasnoyarsk, Russian Federation
}

\begin{abstract}
We discuss the mechanisms of unconventional superconductivity and superfluidity in 3D and 2D fermionic systems with purely repulsive interaction at low densities. We construct phase diagrams of these systems and find the areas of the superconducting state in free space, as well as on the lattice in the framework of the Fermi-gas model with hard-core repulsion, the Hubbard model, the Shubin-Vonsovsky model, and the $t-J$ model. We demonstrate that the critical superconducting temperature can be greatly increased in the spin-polarized case or in a two-band situation already at low densities. The proposed theory is based on the Kohn-Luttinger mechanism or its generalizations and explains or predicts anomalous $p$-, $d$-, and $f$-wave pairing in various materials, such as high-temperature superconductors, the idealized monolayer and bilayer of doped graphene, heavy-fermion systems, layered organic superconductors, superfluid ${ }^{3}$ He, spin-polarized ${ }^{3} \mathrm{He}$ mixtures in ${ }^{4} \mathrm{He}$, ultracold quantum gases in magnetic traps, and optical lattices.
\end{abstract}

Keywords: anomalous superconductivity, Kohn-Luttinger mechanism, superfluidity, Fermi gas with repulsion, Hubbard and $t-J$ model, Shubin-Vonsovsky model, graphene monolayer, graphene bilayer

PACS Numbers: 67.85.-d, 74.20.-z, 74.20.Mn, 74.20.Rp, 74.25.Dw, 74.78.Fk, 81.05.ue

Contents

1. Introduction

2. Superconductivity in the model of a Fermi gas with repulsion

3. Superconductivity in the $3 \mathrm{D}$ and $2 \mathrm{D}$ Hubbard model with repulsion

4. Enhancement of the critical temperature in the two-band Hubbard model and in a spin-polarized Fermi gas

5. Nontrivial corrections to the Landau Fermi liquid theory in 2D low-density systems

6. Superconductivity in the ShubinVonsovsky model

7. Superconductivity in the $2 \mathrm{D} t-J$ model 18

8. Superconductivity in an idealized graphene monolayer

9. Enhancement of superconductivity in an idealized graphene bilayer

10.New promising systems with anomalous pairing

1

11.Conclusions

\section{3} of 1 Mbar raises our hopes to move forward from 'high-temperature' to 'room-temperature' superconductors. At the same time, there are many interesting low-temperature superconducting and superfluid systems with anomalous types of pairing and a nontrivial structure of the order parameter. In this review, we consider systems with a low density of fermions in the framework of the nonphonon mechanisms of superconductivity, such as the famous Kohn-Luttinger mechanism and its generalizations, and the exchange mechanisms connected with the antiferromagnetic attraction of spins on neighboring sites, which are topical, in particular, for the $t-J$ model, in which both low and high critical temperatures of the superconductive transition and anomalous $p$-, $d$-, and $f$-wave Cooper pairing can appear.

As is known from textbooks, the conduction electrons in metals, together with the positively charged ions, form a solid-state plasma, which determines the combination of their electric, galvanomagnetic, kinetic, and superconducting properties. The coupling 
between the subsystem of massive positive ions and the subsystem of light fermions leads to the appearance of the electron-phonon interaction, which affects the properties of the electron subsystem. In particular, the effective interaction between electrons in a solid-state plasma can differ significantly from the Coulomb interaction of electrons in the vacuum and can even change sign. This most important effect is the basis of the electron-phonon mechanism of Cooper instability in standard superconductors [3].

It is obvious that the role of the mediator (coupling to which initiates the renormalization of the Coulomb interaction) can be played by any other subsystem. It is only necessary that the interaction of the electron gas with this subsystem lead to polarization effects that cause the generation of electrons and holes in the vicinity of the Fermi surface. Notably, in many theoretical studies on high-temperature superconductors, collective excitations of the subsystem of localized spins of copper ions serve as such a mediator. This, in particular, determines the spin-fluctuation mechanism of Cooper instability, which leads to the formation of a superconducting phase with $d$-type symmetry of the order parameter.

Within the formalism of the secondary quantization of fermions, the operator of Coulomb interaction of electrons contains terms that in the higher orders of the perturbation theory initiate polarization contributions to the ground-state energy, which also leads to the renormalization of the Coulomb interaction of electrons. Therefore, the effective interaction of electrons in such a metal can differ significantly from the electron-electron interaction in the vacuum. This makes topical the problem (first formulated by Anderson [4]) of such a renormalization of Coulomb interaction under which the effective electron-electron interaction in a substance would have an attractive rather than repulsive nature. In other words, the problem consists in searching for conditions under which the above-mentioned polarization effects in the electron plasma of a metal would lead to a change in the sign of the resulting interaction between the electrons. From the mathematical standpoint, the problem reduces to calculating the effective pairwise interaction of electrons with multiparticle effects in the electron ensemble taken into account. No less important, according to Anderson, is the problem of explaining the unconventional properties of the normal state of many strongly correlated electronic systems at temperatures higher than the critical temperature, especially in the pseudogap state.

In recent decades, considerable progress has been achieved in experimental and theoretical studies of superconductive systems with a nonphonon nature of the Cooper pairing and with a complex, nontrivial structure of the order parameter. The first experimentally discovered systems with unconventional triplet $p$-wave pairing (the total spin of the Cooper pair $S_{\text {tot }}=1$ and the orbital momentum of the relative motion of the pair $l=1$ ) were the superfluid $\mathrm{A}$ and $\mathrm{B}$ phases of ${ }^{3} \mathrm{He}$ with low critical temperatures, $T_{c} \sim 1 \mathrm{mK}$. Another example of systems in which the $p$-wave pairing is realized are ${ }^{6} \mathrm{Li}_{2}$ and ${ }^{40} \mathrm{~K}_{2}$ molecules in magnetic traps in the regime of the $p$-wave Feshbach resonance with ultralow critical temperatures: $T_{c} \sim 10^{-6}-10^{-7} \mathrm{~K}[5,6]$. It is assumed that unconventional $p$-wave pairing with critical temperatures $T_{c} \sim 0.5-1 \mathrm{~K}$ is realized in some heavy-fermion intermetallic compounds, such as $\mathrm{U}_{1-x} \mathrm{Th}_{x} \mathrm{Be}_{13}$ and $\mathrm{UNl}_{2} \mathrm{Al}_{3}$, with large effective masses $m^{*} \sim 100-200 m_{e}$ [7, 8]. Frequently, the $p$-wave pairing is mentioned in connection with organic superconductors, such as $\alpha-(\text { BEDT-TTF })_{2} \mathrm{I}_{3}$ with $T_{c} \sim 5 \mathrm{~K}[9]$. Finally, $p$-wave pairing with $T_{c} \sim 1 \mathrm{~K}$ is apparently realized in the ruthenates $\mathrm{Sr}_{2} \mathrm{RuO}_{4}$ [10, 11], and it cannot be excluded in the layered dichalcogenides $\mathrm{CuS}_{2}-\mathrm{CuSe}_{2}$ or the semimetals and semimetallic superlattices InAs- $\mathrm{GaSb}, \mathrm{PbTe}-$ SnTe [12, The heavy-fermionic intermetallic compound $\mathrm{UPt}_{3}$ with $m^{*} \sim 200 m_{e}$ and $T_{c} \sim 0.5 \mathrm{~K}$, as well as a large class of high-temperature cuprate superconductors with critical temperatures from $T_{c}=36 \mathrm{~K}$ (for the lanthanum-based compounds) to $T_{c}=160 \mathrm{~K}$ (obtained in mercury-based superconductors under pressure), are related to unconventional superconductors with the singlet $d$-wave pairing $\left(S_{\text {tot }}=0, l=2\right)$. Finally, in connection with the problems of applied superconductivity, it is also necessary to mention new multiband superconductors with a more conventional $s$-wave pairing, such as $\mathrm{MgB}_{2}$ [13], and the recently discovered superconductors based on iron arsenide [14] and the $\mathrm{H}_{2} \mathrm{~S}$ and $\mathrm{PoH}_{2}$ metallic compounds already noted above [15.

Along with the problems of Cooper pairing in the above-mentioned electron systems, also of significant interest is the search for fermionic superfluidity in three-dimensional (3D) and two-dimensional (2D) (thin films, submonolayers) solutions of ${ }^{3} \mathrm{He}$ in ${ }^{4} \mathrm{He}$ [16 18] and for superconductivity in doped graphene [19], which are problems that have still not been solved experimentally. These systems are among the most promising ones from the standpoint of the experimental and theoretical description of a wide class of physical phenomena and of the nature of multiparticle correlations in them.

Notably, submonolayers of ${ }^{3} \mathrm{He}$ adsorbed on different substrates, such as a solid substrate or the free surface of superfluid ${ }^{4} \mathrm{He}$, with the variation of the 
particle density in wide ranges, make it possible to realize different regimes in the system - from the ultrararefied Fermi gas to strongly correlated Fermi systems [20]. This makes the solutions ideal objects for the development and verification of different methods of the Fermi liquid theory. The unbalanced (spinpolarized) ultracold Fermi gases in 3D and especially in 2D magnetic traps are also very promising [21,22].

Of significant interest from both the fundamental and applied standpoints is graphene, because of its unique electronic properties [23, 24]. Near the Fermi level, the electrons in graphene have a linear dispersion, and the energy gap between the valence band and the conduction band is absent; therefore, the electrons in graphene can be described by a 2D Dirac equation for massless charged quasiparticles 25. The properties of these quasiparticles, such as their twodimensionality, the spinor nature of their spectrum, the zero mass, and the absence of the gap in the spectrum, lead to a number of phenomena that have no counterparts in other physical systems [26].

The above-mentioned studies have stimulated an intensive search for alternative mechanisms of pairing based on strong correlations in the Fermi liquid. The most promising in this respect are the KohnLuttinger mechanism [27, proposed in 1965, and its generalizations (see, e.g., [28]). The Kohn-Luttinger mechanism assumes the transformation of the pure repulsive interaction of two particles in the vacuum in the presence of a fermionic background into an effective attraction in the substance in the channel with a nonzero orbital angular momentum of the pair.

This review is devoted to the description of basic results obtained in recent decades concerning KohnLuttinger superconductivity in repulsive Fermi systems and its generalizations as well as the exchange mechanisms of superconductivity in the generalized $t-J$ model.

\section{Superconductivity in the model of a Fermi gas with repulsion}

The basic model for studying the nonphonon mechanisms of superconductivity in low-density electron systems is the model of a Fermi gas. In the case of a Fermi gas with attraction, the scattering length $a$ is negative $(a<0)$, which results in a traditional $s$-wave pairing (total spin $S=0$, orbital angular momentum $l=0$ ) with the critical temperature

$$
T_{c}^{s} \approx 0.28 \varepsilon_{F} \exp \left(-\frac{\pi}{2|a| p_{F}}\right) .
$$

where $\varepsilon_{F}$ is the Fermi energy and $p_{F}$ is the Fermi momentum.

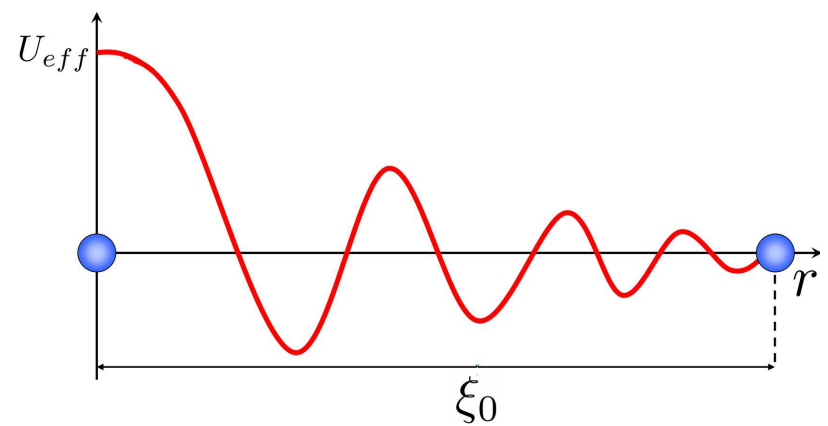

Fig. 1. Friedel oscillations in the effective interaction of two particles as a result of the polarization of the fermionic background, where $\xi_{0}$ is the coherence length of the Cooper pair 32 .

This result was obtained in Ref. 29] soon after the appearance of the Bardeen-Cooper-Schrieffer (BCS) theory [3]. Result (1) differs from the classical formula given in [3] by the presence of a quantity $0.28 \varepsilon_{F}$ in the preexponential factor instead of the Debye frequency $\omega_{D}$ typical for the phonon models in conventional superconductors.

In the model of a Fermi gas with repulsion, the scattering length $a$ is positive $(a>0)$ and the superconductivity corresponds to the Kohn-Luttinger mechanism in the low-temperature region. The physical reason for this consists in the effective interaction of quasiparticles, which arises as a result of the polarization of the fermionic background. Due to a sharp boundary existing in the momentum space which is equal to the diameter of the Fermi sphere $2 p_{F}$ and separates the occupied states from the empty ones, the effective interaction of quasiparticles that are located on the Fermi level does not decrease exponentially, but has an oscillating form (Friedel oscillations [30,31])

$$
U_{\mathrm{eff}}(r) \sim \frac{\cos \left(2 p_{F} r\right)}{\left(2 p_{F} r\right)^{3}} .
$$

If the distance between two electrons in a Cooper pair is relatively large, effective interaction (2) in the coordinate space has a large number of maxima and minima (Fig. 1). Then the integral effect determined by the averaging over the potential relief of Friedel oscillations can, in principle, lead to an effective attraction and the appearance of superconductivity in the system.

The first to advocate this mechanism of superconductivity were Kohn and Luttinger [27], who considered 3D repulsive Fermi systems. They showed that the effective interaction in the first two orders of the perturbation theory in the gas parameter (more precisely, in the scattering length $a$ ) is described by the sum of the five diagrams shown in Fig. 2, The first 
diagram corresponds to the bare interaction of two electrons in the Cooper channel. The next four diagrams (Kohn-Luttinger diagrams) are due to secondorder processes and take into account the polarization effects of the filled Fermi sphere. In the case of a short-range potential, the contribution to the effective interaction is determined only by the fourth exchange diagram, and in the first two orders of the perturbation theory, the expression for $U_{\text {eff }}$ can be written as

$$
U_{\text {eff }}(\mathbf{p}, \mathbf{k})=\frac{4 \pi a}{m}+\left(\frac{4 \pi a}{m}\right)^{2} \Pi(\mathbf{p}+\mathbf{k}),
$$

where $4 \pi a / m$ is the pseudopotential, which corresponds to the wavy line in Fig. 2, and $\Pi(\mathbf{p}+\mathbf{k})$ is the static polarization operator, which is described by the standard Lindhard function 34, 35.

$$
\Pi(\mathbf{p}+\mathbf{k})=\frac{1}{N} \sum_{\mathbf{p}_{1}} \frac{n_{F}\left(\varepsilon_{\mathbf{p}_{1}-\mathbf{p}-\mathbf{k}}\right)-n_{F}\left(\varepsilon_{\mathbf{p}_{1}}\right)}{\varepsilon_{\mathbf{p}_{1}}-\varepsilon_{\mathbf{p}_{1}-\mathbf{p}-\mathbf{k}}} .
$$

This operator is responsible for the charge screening in the electron plasma in metals. The plus sign in the argument of the polarization operator is due to the so-called crossing, which, in the case of short-range repulsion, distinguishes the exchange diagram from the true polarization loop, which contains the argument $\mathbf{p}-\mathbf{k}$. In the absence of a lattice, $\varepsilon_{\mathbf{p}}=\frac{\mathbf{p}^{2}}{2 m}$ is the energy spectrum,

$$
n_{F}(x)=\left(\exp \left(\frac{x-\mu}{T}\right)+1\right)^{-1}
$$

is the Fermi-Dirac distribution function, and $\mu$ is the chemical potential.

It was noted in the early work of Migdal [36] and Kohn [37] that at low temperatures $\left(T \ll \varepsilon_{F}\right)$, the polarization operator contains, apart from a regular part, a singular part - the so-called Kohn anomaly, which in the $3 \mathrm{D}$ case has the form

$$
\Pi_{\text {sing }}(\widetilde{q}) \sim\left(\widetilde{q}-2 p_{F}\right) \ln \left|\widetilde{q}-2 p_{F}\right|,
$$

where we have $\widetilde{q}=|\mathbf{p}+\mathbf{k}|$ in the cross-channel. In the coordinate space, the singular part of $\Pi_{\text {sing }}$ leads to Friedel oscillations (2) in the effective interaction (see Fig. (1).

Thus, the purely repulsive short-range potential between two particles in vacuum induces an effective interaction in the electron gas in a metal with the competition between repulsion and attraction. It turns out that the singular part in $U_{\text {eff }}$ favors attraction, ensuring a contribution that always exceeds the repulsive contribution caused by the regular part of

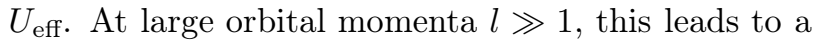
superconducting instability with the critical temperature $T_{c l} \sim \varepsilon_{F} \exp \left(-l^{4}\right)$. In this case, the conventional
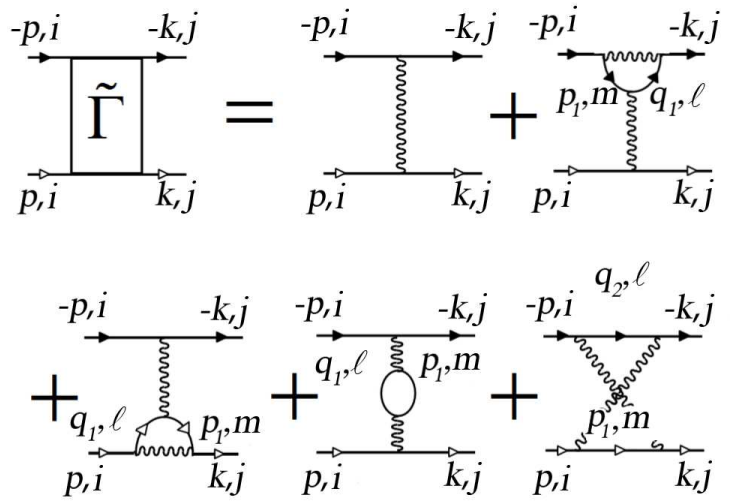

Fig. 2. Diagrams of the first and second order for the effective interaction of electrons $U_{\text {eff }}$. The solid lines with light (dark) arrows correspond to the Green functions of electrons with the spin projections $+\frac{1}{2}\left(-\frac{1}{2}\right) ; \mathbf{q}_{1}=\mathbf{p}_{1}+\mathbf{p}-\mathbf{k}$ и $\mathbf{q}_{2}=\mathbf{p}_{1}-\mathbf{p}-\mathbf{k}$. The wavy lines correspond to the bare interaction. In the case of a Fermi gas (see Section [2), the Hubbard model (see Section \$3), and the Shubin-Vonsovsky model (see Section $\$$ ), the indices in the diagrams are $i=j=l=m=1$. In the case of a graphene monolayer (see Section $\$$ ) $i=j=1$, and $l, m=1,2$. In the case of a graphene bilayer (see Section $\Phi$ ) $i, j=1,2$ and $l, m=1,2,3,4$ 33.

singlet pairing in the $s$-wave channel $\left(S_{\mathrm{tot}}=0, l=0\right)$ is suppressed by the short-range Coulomb repulsion caused by the main maximum in $U_{\text {eff }}$ (see Fig. 1), and superconductivity is realized at large orbital momenta, $l \gg 1$. We note that at $l \neq 0$, the role of the main maximum is weakened by the centrifugal potential, which improves the conditions for the appearance of superconductivity in channels with anomalous pairing.

From Ref. [27, a nontrivial conclusion followed that no Fermi systems exist in the normal state at a zero temperature; any $3 \mathrm{D}$ electronic system with a purely repulsive interaction between particles is unstable with respect to the transition to the superconducting state with a large orbital angular momentum of the relative motion of a Cooper pair $(l \gg 1)$. However, the estimates carried out in [27] for the critical temperature in electronic systems in metals with realistic parameters and for superfluid helium at $l=2$ gave very low values of the critical temperature: $T_{c} \sim 10^{-16} \mathrm{~K}$ for ${ }^{3} \mathrm{He}$ and $T_{c} \sim 10^{-11} \mathrm{~K}$ for metallic plasma. The low value of $T_{c}$ was one of the reasons why the Kohn-Luttinger mechanism was not popular among researchers for a sufficiently long period and was unjustly forgotten.

Later on, in Refs [38, 39], it was shown that the 
temperature of the superconducting transition in 27 ] was underestimated because of the utilization of an asymptotic expression for large values of the orbital angular momentum, $l \gg 1$. In reality, at $l=1$, an exact analytic calculation shows that the contributions to $U_{\text {eff }}$ that correspond to the attraction of quasiparticles dominate over the repulsive contributions. As a result, the repulsive 3D Fermi gas is unstable with respect to the superconducting transition with the triplet $p$-wave pairing at the critical temperature [38, 41]

$$
\begin{aligned}
T_{c 1} & \approx \varepsilon_{F} \exp \left(-\frac{5 \pi^{2}}{4(2 \ln 2-1)\left(a p_{F}\right)^{2}}\right) \\
& =\varepsilon_{F} \exp \left(-\frac{13}{\lambda^{2}}\right)
\end{aligned}
$$

where $\lambda=\frac{2 a p_{F}}{\pi}$ is the effective 3D Galitskii gas parameter [42, We note that for $l=1$, the contribution from the Kohn anomaly only increases the value of $T_{c 1}$, but does not play a decisive role in the appearance of the triplet superconductivity itself.

It was demonstrated in Ref. 43 the critical temperature of a superfluid transition can be substantially increased already at low fermionic densities by placing the system of neutral Fermi particles into a magnetic field or by creating spin polarization $\left(n_{\uparrow}>n_{\downarrow}\right)$. This occurs because the paramagnetic suppression of the superconductivity (which takes place for $s$-wave pairing) is absent in the $p$-wave channel for the so-called $A_{1}$ phase and the increase in $T_{c}$ is possible due to the enhancement of the effective interaction and the changes in the character of the Kohn anomaly. The highest critical temperatures then correspond to the $A_{1}$ phase, where the Cooper pair is formed by two spins up, and the effective interaction for them is prepared by two spins down. In this case, $T_{c}$ is a function of the ratio of the density of spin-up particles to the density of spin-down particles, $n_{\uparrow} / n_{\downarrow}$, or more precisely of the spin polarization $\alpha=\frac{n_{\uparrow}-n_{\downarrow}}{n_{\uparrow}+n_{\downarrow}}$.

In the case of a repulsive $2 \mathrm{D}$ electron gas, in the first two orders of the perturbation theory in the gas parameter, the effective interaction takes the form [44,45]

$$
U_{\mathrm{eff}}(\mathbf{p}, \mathbf{k})=\frac{4 \pi}{m} f_{0}+\left(\frac{4 \pi}{m} f_{0}\right)^{2} \Pi(\mathbf{p}+\mathbf{k})
$$

where $f_{0}=\frac{1}{2 \ln \left(p_{F} r_{0}\right)}$ is the Bloom $2 \mathrm{D}$ gas parameter [46] and $\Pi(\mathbf{p}+\mathbf{k})$ is the $2 \mathrm{D}$ polarization operator in the cross channel.
In the $2 \mathrm{D}$ situation, the effective interaction in the coordinate space also contains Friedel oscillations:

$$
U_{\text {eff }}(r) \sim f_{0}^{2} \frac{\cos \left(2 p_{F} r\right)}{\left(2 p_{F} r\right)^{2}},
$$

which are even much stronger than in the $3 \mathrm{D}$ case. But in the momentum space, the 2D Kohn anomaly has one-sided character [47,

$$
U_{\mathrm{eff}}^{\text {sing }}(\widetilde{q}) \sim f_{0}^{2} \operatorname{Re} \sqrt{\widetilde{q}-2 p_{F}}=0
$$

for $\widetilde{q}=|\mathbf{p}+\mathbf{k}| \leq 2 p_{F}$, and, therefore, is ineffective for the problem of superconductivity (in which $\widetilde{q} \leq 2 p_{F}$ ). Thus, the 2D Fermi gas with repulsion remains in the normal state at least in the first two orders of the perturbation theory in the gas parameter $f_{0}$. Nevertheless, it was shown in 44 that the superconducting $p$-wave pairing appears in the next (third) order of the perturbation theory in $f_{0}$, in which, for the singular contribution to the effective interaction, the expression under the square root in (9) reverses sign:

$$
U_{\mathrm{eff}}^{\text {sing }}(\widetilde{q}) \sim f_{0}^{3} \operatorname{Re} \sqrt{2 p_{F}-\widetilde{q}} .
$$

In this case, an exact calculation 48 of the critical temperature taking all irreducible third-order diagrams into account, yields

$$
T_{c} \sim \varepsilon_{F}\left(-\frac{1}{6.1 f_{0}^{3}}\right) .
$$

For the submonolayers of ${ }^{3} \mathrm{He}$ on the surface of superfluid films of ${ }^{4} \mathrm{He}$ [9], the temperature of the superconducting transition is estimated as $10^{-4} \mathrm{~K}$ [4, 48, for the maximal densities at which the Fermi-gas description is still applicable, and this estimate is quite reasonable for experimental observation.

\section{Superconductivity in the $3 \mathrm{D}$ and $2 \mathrm{D}$ Hubbard model with repulsion}

In connection with the discovery of hightemperature superconductivity [50, the Hubbard model 51] acquired substantial popularity as one of the basic models for describing the anomalous properties of cuprates. The Hubbard model is a particular case of the general model of interacting electrons, whose band structure is described within the strong-coupling approximation and is the minimal model that accounts for band electron motion in the metal as well as the strong electron-electron interaction 52 56]. This model is of special importance in the description of narrow-band metals 48. The Hamiltonian of the Hubbard model on a lattice has the form 


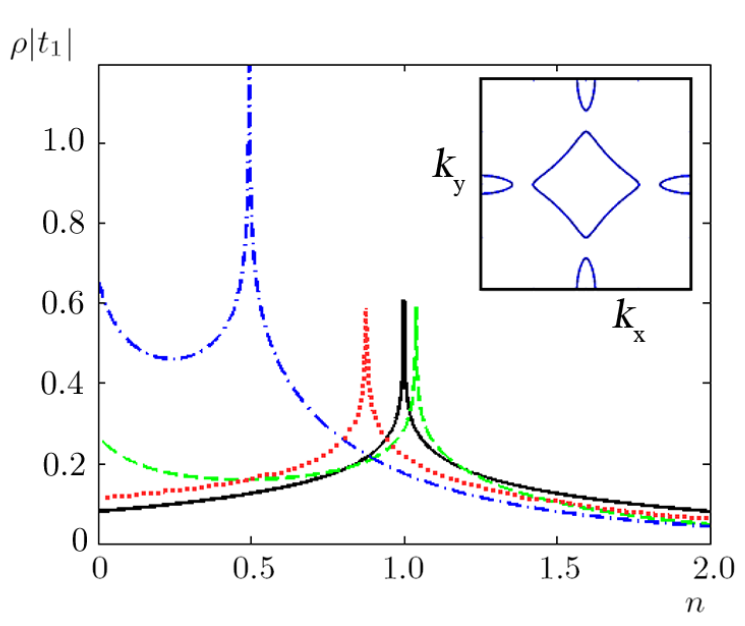

Fig. 3. Modification of the density of electronic states and the shift of the Van Hove singularity in the Hubbard model on a square lattice upon a change in the hopping integrals: $t_{2}=t_{3}=0$ (solid curve), $t_{2}=0.15, t_{3}=0$ (dotted curve), $t_{2}=0.15, t_{3}=0.1$ (dashed curve), $t_{2}=0.44, t_{3}=0$ (dashed-dotted curve). The inset shows the formation of a multisheet Fermi contour at $t_{2}=0.44, t_{3}=-0.1, \mu=2$ (all the parameters are given in the units of $\left|t_{1}\right|$ ) [32.

$\hat{H}^{\prime}=\sum_{f \sigma}(\varepsilon-\mu) n_{f \sigma}+\sum_{f m \sigma} t_{f m} c_{f \sigma}^{\dagger} c_{m \sigma}+U \sum_{f} n_{f \uparrow} n_{f \downarrow}$,

where $c_{f \sigma}^{\dagger}\left(c_{f \sigma}\right)$ is the operator of creation (annihilation) of an electron with a spin projection $\sigma= \pm 1 / 2$ at a site $f ; \varepsilon$ is the on-site energy of the electron; $\mu$ is the chemical potential of the system; $n_{f}=\sum_{\sigma} n_{f \sigma}=$ $\sum_{\sigma} c_{f \sigma}^{\dagger} c_{f \sigma}$ is the operator of the number of particles on the site $f$; the matrix element $t_{f m}$ stands for electron hoppings from site $f$ to site $m$; and $U$ is the parameter of the Coulomb interaction of two electrons that are located on the same site and have opposite spin projections (Hubbard repulsion).

Since extensive experimental data have indicated that the basic dynamics of Fermi excitations in cuprates is developed in the $\mathrm{CuO}_{2}$ planes, the nonphonon mechanisms of superconductivity were mainly based on the 2D Hubbard model on a simple square lattice. In the momentum space, the Hamiltonian of the model has the form

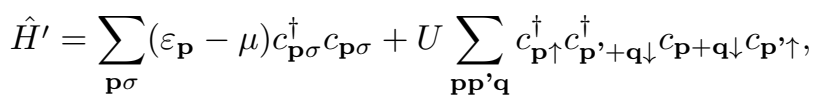

where the energy of an electron, including distant hoppings, which are determined by the parameters $t_{2}$ and $t_{3}$, is given by

$$
\begin{aligned}
\varepsilon_{\mathbf{p}} & =2 t_{1}\left(\cos p_{x} a+\cos p_{y} a\right)+4 t_{2} \cos p_{x} a \cos p_{y} a \\
& +2 t_{3}\left(\cos 2 p_{x} a+\cos 2 p_{y} a\right) .
\end{aligned}
$$

where $a$ is the lattice constant (intersite distance).

We note that when simulating electron spectrum (14) and constructing the phase diagram of the superconducting state in the Hubbard model, going beyond the framework of the nearest-neighbor approximation $\left(t_{2} \neq 0, t_{3} \neq 0\right)$ becomes essential. This is because the leading contribution to the effective coupling constant comes from the interaction of electrons that are located near the Fermi surface, whose geometry depends on the structure of the energy spectrum. An important role is also played by the fact that account for distant hoppings shifts the Van Hove singularity in the density of electronic states from the position at half-filling $(n=1)$ into the region of smaller or higher electronic densities (Fig. 31). We note that the introduction of hoppings to the third coordination sphere of the square lattice, $t_{3} \neq 0$, can lead to a qualitative change in the geometry of the Fermi surface, which connected with the formation of a multisheet Fermi contour (see the inset in Fig. (3).

Thus, an account for distant hoppings can lead to a modification of the phase diagram that determines the regions of the realization of the superconducting states with different types of the order parameter symmetry.

In the Hubbard model, the perturbation theory can be constructed in two limiting cases: (1) the Born weak-coupling approximation, $U \ll W(W=2 z t$ is the bandwidth; $z$ is the number of nearest neighbors) and an arbitrary electron density, $0<n<1$; and (2) the strong-coupling approximation $U \gg W$ at low electron density, $n \ll 1$. The utilization of the weakcoupling approximation $U \ll W$ in the analysis of the feasibility of Kohn-Luttinger superconducting pairing allows us to calculate $U_{\text {eff }}$ for the Cooper channel at all the densities $0<n \leq 1$ by restricting ourselves to the second order diagrams in the interaction $U$ (see Fig. (2). In the opposite limit of strong coupling, $U \gg W$, the restriction to first- and second-order diagrams is justified only in the region of low electron density $n<<1$, where the Galitskii-Bloom Fermi-gas expansion is valid [42,46].

In one of the first studies [57, the authors analyzed the conditions for the realization of the Kohn-Luttinger superconductivity in the 2D Hubbard model with Hamiltonian (13) in the weak-coupling limit $U \ll W$, in the nearest-neighbor approximation $\left(t_{2}=t_{3}=0\right)$ at low electron densities $\left(p_{F} a \ll 1\right)$. In this case, the following expansion is valid for the electron spectrum: 


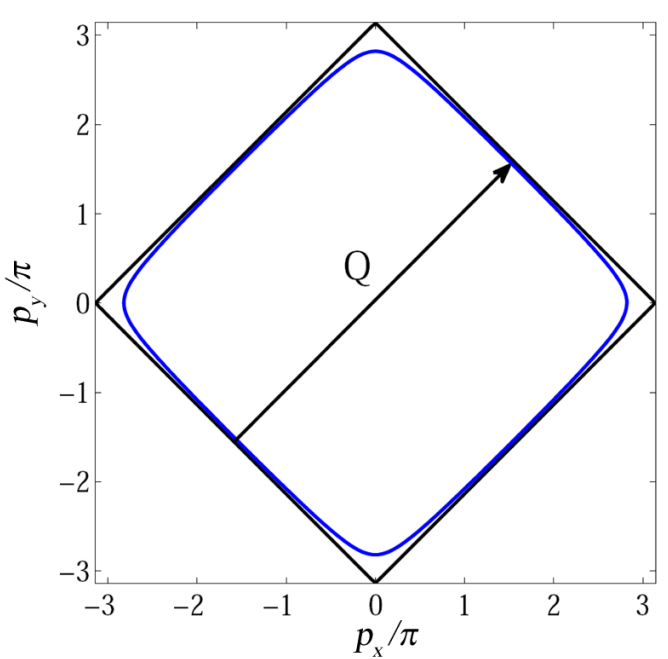

Fig. 4. Fermi surface for a nearly half filling $(n \approx 1)$ in the 2D Hubbard model on a square lattice: $\mathbf{Q}=$ $(\pi / a, \pi / a)$ is the nesting vector 32 .

$$
\begin{aligned}
\varepsilon_{\mathbf{p}}-\mu & =2 t_{1}\left(\cos p_{x} a+\cos p_{y} a\right)-\mu \\
& \approx \frac{p^{2}-p_{F}^{2}}{2 m}-\frac{\left(p_{x}^{4}+p_{y}^{4}\right) a^{2}}{24 m}+\frac{\left(p_{x}^{6}+p_{y}^{6}\right) a^{4}}{720 m} .
\end{aligned}
$$

where $m=1 / 2 t_{1} a^{2}$ is the band mass. It can be seen that in the chosen approximation, the bare spectrum of electrons at $p_{F} a \ll 1$ almost coincides with the spectrum of a free Fermi gas, and the Hubbard Hamiltonian is equivalent to the Hamiltonian of a weakly nonideal Fermi gas with short-range repulsion between the particles [58]. To determine the possibility of the superconducting pairing in this approximation, the effective bare vertex for the Cooper channel was calculated in first two orders of perturbation theory:

$$
U_{\mathrm{eff}}(\mathbf{p}, \mathbf{k})=U+U^{2} \Pi(\mathbf{p}+\mathbf{k})
$$

where $\Pi(\mathbf{p}+\mathbf{k})$ is polarization operator (4). To solve the problem of superconducting pairing, the function $U_{\text {eff }}(\mathbf{p}, \mathbf{k})$ was expanded in a series with eigenfunctions of the irreducible representations of the $C_{4 v}$ symmetry group of the square lattice (see Section 6 .), and then the sign of the expressions for $U_{\text {eff }}^{\gamma}$ was analyzed for each representation $\gamma$. As a result, it was shown that the 2D electron system described by the Hubbard model for small filling and $U \ll W$ is unstable towards the superconducting pairing with the $d_{x y}$-type symmetry of the order parameter $\Delta(\phi) \sim \sin (4 m+2) \phi$, where the integer $m$ satisfies the condition $m \in[0, \infty)$.

The weak-coupling limit $U \ll W$ in the 3D and 2D Hubbard model near the half-filling, $n \sim 1$, was analyzed in 5961 . In the $2 \mathrm{D}$ case [61, in the nearest- neighbor approximation at $n \approx 1$, the electron spectrum becomes quasihyperbolic

$$
\varepsilon_{\mathbf{p}} \approx \pm \frac{p_{x}^{2}-p_{y}^{2}}{2 m}
$$

near the corner points $(0, \pm \pi)$ and $( \pm \pi, 0)$ at which the Fermi surface almost touches the Brillouin zone (Fig. 4). As it is well known, the density of electronic states has a logarithmic singularity in these regions near the Van Hove filling, namely $\rho(E) \sim \ln \frac{t}{|\mu|}$, where $|\mu| \ll t$ is the modulus of the chemical potential near the half-filling. It can be seen from Fig. 4 that there are almost flat regions of the Fermi surface, which satisfy the condition for ideal nesting in the exactly half-filled case $(n=1)$ :

$$
\varepsilon_{\mathbf{p}+\mathbf{Q}}=-\varepsilon_{\mathbf{p}}
$$

where $\mathbf{Q}=(\pi / a, \pi / a)$ is the nesting vector for the $2 \mathrm{D}$ square lattice. In these regions, the polarization operator reads $\Pi(\mathbf{Q}) \sim \ln ^{2} \frac{t}{|\mu|}[61,62$, where one logarithmic factor comes from the density of states and the other one is due to the Kohn anomaly. The following quantity serves here as the parameter of the perturbation theory in the $2 \mathrm{D}$ weak-coupling limit:

$$
f_{0}=\frac{U}{8 \pi t} \ll 1,
$$

and in the second order of the perturbation theory in $f_{0}$, the expression for the effective interaction takes the form

$$
U_{\text {eff }} \sim f_{0}+f_{0}^{2} \ln ^{2} \frac{t}{|\mu|}
$$

Since the expression for the Cooper loop $L$ at $n \approx 1$, apart from the usual Cooper logarithm, also contains a logarithm due to the Van Hove singularity, we have

$$
L\left(\xi_{\mathbf{p}}\right)=\frac{1}{N} \sum_{\mathbf{p}} \frac{\tanh \left(\xi_{\mathbf{p}} / 2 T\right)}{2 \xi_{\mathbf{p}}} \sim \ln \frac{\mu}{T} \ln \frac{t}{|\mu|},
$$

where $\xi_{\mathbf{p}}=\varepsilon_{\mathbf{p}}-\mu$. Therefore, the expression for the critical temperature with the order parameter of the

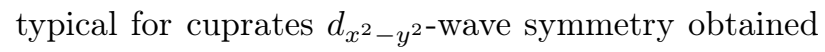
in Ref. [61] in the leading logarithmic approximation has the form

$$
f_{0}^{2} \ln ^{3} \frac{t}{|\mu|} \ln \frac{\mu}{T_{c}} \sim 1
$$

or

$$
T_{c}^{d_{x^{2}-y^{2}}} \sim \mu \exp \left(-\frac{1}{f_{0}^{2} \ln ^{3} \frac{t}{|\mu|}}\right) .
$$

It can be seen from expression (23) that the denominator in the right-hand side, in spite of the low value 
of $f_{0}^{2}$ at $f_{0} \ll 1$, increases substantially due to the large value of $\ln ^{3} \frac{t}{|\mu|} \gg 1$.

The results in [57] on the realization of $d_{x y}$-wave

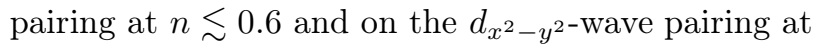
$n \sim 1$ [59 61] in the weak-coupling limit were subsequently confirmed by other authors. In 63], a phase diagram of the superconducting state of the 2D Hubbard model was constructed at small and intermediate occupation numbers, which shows how the results of the competition of different types of the order parameter symmetry to depend on the value of the parameter $t_{2}$ of the electron hoppings to the next-nearestneighbor sites. The phase diagram obtained in the second order of the perturbation theory shows that at $t_{2}=0$ in the region of the low electron densities, $0<n<0.52$, superconductivity with the $d_{x y}$-type symmetry of the order parameter is realized. In the range of $0.52<n<0.58$, the ground state corresponds to the phase with the $p$-wave pairing, and at $n>0.58$ the $d_{x^{2}-y^{2}}$-wave pairing appears. Similar results were obtained in 64 in the framework of the renormalization group approach.

In vicinity of the half-filling, $0.95<n<1$, where strong competition between superconductivity and antiferromagnetism is observed, the problem of Cooper instability was examined in Refs 662, 65, 66. In these studies, the so-called parquet diagrams were summed up and at $\mu \sim T_{c}$ the relation

$$
f_{0}^{2} \ln ^{4} \frac{t}{|\mu|} \sim f_{0}^{2} \ln ^{4} \frac{t}{T_{c}} \sim 1 .
$$

was obtained. This yields an elegant estimate for the maximal critical temperature:

$$
T_{c}^{d_{x^{2}-y^{2}}} \sim t \exp \left(-\frac{\text { const }}{\sqrt{f_{0}}}\right) .
$$

The maximal critical temperature of the superconducting transition in the 2D Hubbard model was obtained in Ref. 67] in the regime $U / W \sim 1$ at optimal electron concentrations $n \sim 0.8-0.9$. According to the estimate obtained in [67, the critical temperature can reach the values $T_{c}^{d_{x^{2}-y^{2}}} \approx 100 \mathrm{~K}$, which is quite reasonable for optimally doped cuprate superconductors.

\section{Enhancement of the critical temperature in the two-band Hubbard model and in a spin-polarized Fermi gas}

Along with the possibility to enhance the anomalous superconductivity by applying a magnetic field to a system of neutral Fermi particles as described in
Section 2 , there is also another possibility to increase $T_{c}$ significantly already at a low electron density. It is related to the two-band situation 68, or a multilayer system with geometrically separated layers. In this case, the role of spins-up is played by the electrons of the first band (or layer), and the role of spins-down by the electrons of the second band (or layer). The coupling between electrons of two bands is achieved by means of the interband Coulomb interaction $U_{12} n_{1} n_{2}$. As a result, the following exciton-type mechanism of superconductivity becomes possible: the electrons of one band form a Cooper pair via polarization of the electrons of the other band [68 70]. In this case, the role of spin polarization is played by the relative filling of two bands $n_{1} / n_{2}$.

We now examine the two-band Hubbard model with one broad band and one narrow band 69, 70, which accordingly contains 'heavy' $\left(n_{1}=n_{H}\right)$ and 'light' $\left(n_{2}=n_{L}\right)$ electrons. This model is a natural generalization of the well-known Falikov-Kimball model 71 for systems with a mixed valence; however, it exhibits a richer physics in view of the presence of a finite bandwidth of heavy electrons instead of a localized level. In the Hubbard model with one narrow band, the effective interaction, as it was shown in Refs 69,70,72,73, is determined mainly by the interband Coulomb repulsion of heavy and light electrons $U_{12}=U_{H L}$. The corresponding critical temperature of the superconducting transition depends nonmonotonically on the relative filling of the bands $n_{H} / n_{L}$ and exhibits a wide and clearly pronounced maximum at $n_{H} / n_{L}=4$ in the $2 \mathrm{D}$ case. The maximal critical temperature can be expressed as

$$
T_{c \max }=T_{c 1}\left(\frac{n_{H}}{n_{L}} \approx 4\right)=\varepsilon_{F} \exp \left(-\frac{1}{2 f_{0}^{2}}\right),
$$

and corresponds to the triplet $p$-wave pairing of heavy particles via the polarization of light particles. In the Born weak-coupling approximation, the effective gas parameter depends linearly on the interband Coulomb repulsion and on the square root of the product of the masses 68]:

$$
f_{0}=\frac{\sqrt{m_{H} m_{L}}}{2 \pi} U_{H L}
$$

In the opposite limit of strong coupling [69,70,

$$
f_{0}=\sqrt{\frac{m_{H}}{m_{L}}} \frac{1}{\ln \left[1 /\left(p_{F} a\right)^{2}\right]} .
$$

In the so-called unitary limit of the strongly screened Coulomb interaction $\left(f_{0} \rightarrow 1 / 2\right)$ and of the strong electron-polaron effect, $m_{H}^{*} / m_{L} \sim\left(m_{H} / m_{L}\right)^{2}$. Cor- 
respondingly, the maximal critical temperature in this case yields 69, 70,

$$
T_{c 1} \sim \varepsilon_{F H}^{*} \exp \left(-\frac{1}{2 f_{0}^{2}}\right) \sim \varepsilon_{F H}^{*} \exp (-2),
$$

where $\varepsilon_{F H}^{*}$ is the renormalized (strongly narrowed) Fermi energy of heavy particles

$$
\varepsilon_{F H}^{*}=\frac{p_{F H}^{2}}{2 m_{H}^{*}} \sim 30-50 \mathrm{~K} .
$$

Let us stress that in the unitary limit the sharp increase in the effective mass of heavy particles up to $m_{H}^{*} \sim 100 m_{e}$ is caused by the many-body electronpolaron effect [72, 73]. As a result, quite reasonable critical temperatures are obtained for the superconducting $p$-wave pairing: $T_{c} \sim 5 \mathrm{~K}$, typical for uranium-based heavy-fermion compounds, mentioned in the Introduction, $\mathrm{U}_{1-x} \mathrm{Th}_{x} \mathrm{Be}_{13}$ and $\mathrm{UNl}_{2} \mathrm{Al}_{3}$ with large effective masses $m^{*} \sim 100-200 m_{e}[7,8]$ and for organic superconductors.

We note that the electron-polaron effect, which leads to a significant increase in the effective mass in the model, is connected with the nonadiabatic part of the wave function that describes a heavy electron surrounded by a cloud of virtual electron-hole pairs that belong to the band of light electrons [72,73].

The discussed mechanism of superconductivity possibly can be realized in the bismuth- and thalliumbased cuprate superconductors, as well as in the PbTe-SnTe superlattices [12] and dichalcogenides $\mathrm{CuS}_{2}$ and $\mathrm{CuSe}_{2}$ with geometrically separated layers. We note that, in general, the two bands can belong to the same layer or to different layers. It is also reasonable to assume that this mechanism can be fulfilled in the ruthenates $\mathrm{Sr}_{2} \mathrm{RuO}_{4}$ 10 and in the ultracold Fermi gas of ${ }^{6} \mathrm{Li}$ atoms in magnetic traps with a strong imbalance of the hyperfine components (see Section (10).

We note again that in the presence of the band of heavy and light electrons with strongly different masses, $m_{H} \gg m_{L}$, and different densities, $n_{H}>n_{L}$, the critical temperature $T_{c}$ is determined mainly by the pairing of the heavy electrons via the polarization of the light electrons. However, taking into account even an infinitely small Geilikman-Moskalenko-Suhl term [74 78] $K \sum_{\mathbf{p p}^{\prime}} a_{\mathbf{p}}^{\dagger} a_{-\mathbf{p}}^{\dagger} b_{\mathbf{p}^{\prime}} b_{-\mathbf{p}^{\prime}}$ (where $K$ is the parameter of interaction corresponding to the rescattering of the Cooper pairs between the 'heavy' and 'light' bands), leads to the opening of superconducting gaps in both bands at the same critical temperature close to one in (26).

Let us consider the application of this theory to low-temperature physics in more details. We empha- size that the ultracold quantum gases in magnetodipole traps, as well as with the spin-polarized solutions of ${ }^{3} \mathrm{He}$ in ${ }^{4} \mathrm{He}$, especially in the quasi-twodimensional situation (in which the largest increase in the temperature of the triplet $p$-wave pairing occurs), are the excellent systems for verifying the theoretical predictions of [20,41, 43, 68, 70, 79]. Good experimental opportunities for the realization of the 'hightemperature' superfluidity in spin-polarized (imbalanced) Fermi gases in quasi-two-dimensional magnetic traps, in particular, has G E Thomas's group in North Carolina 21. We also note that in the 1990s G Frossati's group in Leyden experimentally obtained a $20 \%$ increase (from 2.5 up to $3.14 \mathrm{mK}$ ) in the critical temperature of the superfluid transition $T_{c 1}^{\uparrow \uparrow}$ in the $A_{1}$ phase of the superfluid ${ }^{3} \mathrm{He}$ in magnetic fields up to $H=15 \mathrm{~T}$ (at a spin polarization of $7 \%$ ) [80,81]. In this case, at the maximum for the critical temperature of the triplet $p$-wave pairing (reached at the spin polarization $\alpha=\frac{n_{\uparrow}-n_{\downarrow}}{n_{\uparrow}+n_{\downarrow}}=48 \%$ ), the theory of [20,43] predicts an increase in $T_{c 1}^{\uparrow \uparrow}$ by a factor of 6.4 in comparison with the nonpolarized case. A similar value for $T_{c 1}^{\uparrow \uparrow}$ at the maximum (with the maximum $T_{c 1}^{\uparrow \uparrow}=5.6 T_{c 1}$ ), but for a $35 \%$ spin polarization, was also predicted in the metamagnetic model in Refs [82,83] in the framework of the so-called $s-p$ approximation for the Landau Fermi liquid theory [84]. We note, however, that the approach in [20,43] in the framework of the enhanced Kohn-Luttinger mechanism of superfluidity is characterized by the only one fitting parameter, the gas parameter $a p_{F}$, and therefore this approach is more model-independent than the one in [82], which uses two fitting parameters that are not connected with each other, namely, the $s$ and $p$ harmonics of the scattering amplitude of quasiparticles on the Fermi surface.

We also note that for solutions of ${ }^{3} \mathrm{He}$ in ${ }^{4} \mathrm{He}$, the theory of [20] and the results in [85,86] predict a phase diagram for the fermionic superfluidity of ${ }^{3} \mathrm{He}$ in the $3 \mathrm{D}$ case with the regions of $s$-wave pairing at small concentration of ${ }^{3} \mathrm{He}$ in the solution $(x=1-2 \%)$ and the regions of $p$-wave pairing at larger concentrations of ${ }^{3} \mathrm{He}(x>2-4 \%)$. The critical temperatures of the $s$-wave pairing in the solutions are maximal at a zero magnetic fields and at $x \sim 1 \%$. According to the estimates in 85, 86, the $s$-wave critical temperature is in the range $10^{-4}-10^{-5} \mathrm{~K}$.

The temperature of the triplet $p$-wave pairing grows sharply in a magnetic field, and at the maximal possible concentration $x_{\max }=9.5 \%$ of ${ }^{3} \mathrm{He}$ in the solution in the field $H \sim 15 \mathrm{~T}$, we have $T_{c 1}^{\uparrow \uparrow} \sim$ $10^{-5}-10^{-6} \mathrm{~K}$. In $2 \mathrm{D}$ solutions of ${ }^{3} \mathrm{He}$ in ${ }^{4} \mathrm{He}$, that is for the submonolayers of ${ }^{3} \mathrm{He}$ at the Andreev lev- 
els [87,88] (appearing similarly to Tamm levels on the free surfaces of thin films of superfluid ${ }^{4} \mathrm{He}[89,90]$ ) or on the grafoils [91, 92, the phase diagram of the solution also contains the regions of $s$ - and $p$-wave pairings.

We emphasize that for $s$-wave pairing in 2D systems, usually two phenomena are realized simultaneously: the pairing of two particles in the real space (with the formation of a molecule or a dimer) and the Cooper pairing in the momentum space. The maximal $T_{c}$ for the $s$-wave pairing, according to [93 95], is in the range of $10^{-3}-10^{-4} \mathrm{~K}$ at the $2 \mathrm{D}$ density $n_{3} \sim 0.01$ of a monolayer [85,86]. At the same time, the temperature of the triplet $p$-wave pairing can be increased in magnetic fields, and at $H \sim 15 \mathrm{~T}$ and the $2 \mathrm{D}$ density $n_{3} \sim 0.05$ of a monolayer, it can become quite accessible experimentally $\left(T_{c 1}^{\uparrow \uparrow} \sim 1 \mathrm{mK}\right.$, according to [20]). The experimental observation of fermionic superfluidity in the 3D solutions and submonolayers of ${ }^{3} \mathrm{He}$ remains a challenge for the 'low-temperature community' [96. A similar phase diagram with the regions of $s$ - and $p$-wave pairings was theoretically predicted in [41] and in [97] for the fermionic isotope of lithium ${ }^{6} \mathrm{Li}$ on the Cooper (BCS) side of the crossover with a region of the Bose-Einstein condensation (BEC) in the regime of the $s$-wave Feshbach resonance.

Let us recall that in the fermionic ${ }^{6} \mathrm{Li}$, the quasiresonance scattering length, which is very large in absolute value $\left(a=-2.3 \cdot 10^{3} \AA\right)$, corresponds to attraction. As a result, in the balanced case $\left(n_{1}=n_{2}\right)$, a singlet $s$-wave pairing with the critical temperature determined by formula (11) is realized for the two hyperfine components of the nuclear spin $I$ that are captured in a magnetic trap. The maximal temperature $T_{c 0}$ in the $3 \mathrm{D}$ case, according to [97], is of the order of $10^{-6} \mathrm{~K}$ at $\varepsilon_{F} \sim 10^{-5} \mathrm{~K}$. However, if the imbalance between the hyperfine components is sufficiently large, such that $\left(n_{1}-n_{2}\right) /\left(n_{1}+n_{2}\right)>T_{c 0} / \varepsilon_{F}$, then, according to the Landau criterion of superfluidity [41, the $s$-wave pairing is totally suppressed. Nevertheless, in this case a $p$-wave pairing can arise if the Cooper pairs (as is the case of the $A_{1}$ phase of superfluid ${ }^{3} \mathrm{He}$ ) are formed by Fermi particles of one hyperfine component while the effective interaction for them is prepared by Fermi particles of another (or others) hyperfine component. In this situation, the maximal critical temperature $T_{c 1}^{\uparrow \uparrow} \sim \varepsilon_{F} \exp \left(-7 / \lambda^{2}\right)$ of the $p$-wave pairing for the optimal ratio of the densities of the hyperfine components, according to 41, can reach $10^{-7} \mathrm{~K}$ at $\varepsilon_{F} \sim 10^{-5} \mathrm{~K}$ and $\lambda \sim 1$.

The effect of $T_{c}$ enhancement in total analogy with the solutions of ${ }^{3} \mathrm{He}$ in ${ }^{4} \mathrm{He}$, for the $p$-wave pairing in the imbalanced gases manifests itself much more strongly and clearly in the quasi-two-dimensional sit- uation 68. Therefore, the experimental achievements obtained in [21, which make it possible to create the quasi-two-dimensional traps and to control their essential parameters (such as the density, temperature, and number of particles on layer-by-layer basis) are very important.

We finally consider one more promising prediction of this theory. It was shown in Ref. [79], that in quasi-two-dimensional (layered) materials in a magnetic field that is strictly parallel to the layer, the appearing vector potential $A_{y}=H z\left(\mathbf{H}=H \mathbf{e}_{x}\right.$, with $x$ and $y$ being the coordinates in the layer) does not change the motion of the electrons and Cooper pairs in the plane of the layer. Therefore, the diamagnetic Meissner effect is completely suppressed. As a result, the electronic monolayer (or the layered system) becomes equivalent to an uncharged (neutral) fermionic layer of ${ }^{3} \mathrm{He}$.

Thus, for low-density quasi-two-dimensional systems, the phase diagram of the superconducting state in a magnetic field parallel to the electronic layer can contain again the regions of conventional $s$-wave pairing in the absence of a magnetic field and the regions of triplet $p$-wave pairing (similar to the $A_{1}$ phase of the superfluid ${ }^{3} \mathrm{He}$ ) in strong magnetic fields, when the $s$-wave pairing is totally suppressed paramagnetically. Moreover, in the magnetic fields $H \sim 15 \mathrm{~T}$ and at low Fermi energies $\left(\varepsilon_{F} \sim 30 \mathrm{~K}\right)$ for sufficiently large degrees of spin polarization of electrons $(\alpha \geq 10 \%)$ the reasonable critical temperatures $\left(T_{c 1}^{\uparrow \uparrow} \sim 0.5 \mathrm{~K}\right)$ can be obtained. Of course, in this case, as in the case of graphene (see Sections 8 and 9), the experimentalists should be very careful analyzing the role of the structural disorder and nonmagnetic impurities, which lead to the isotropization of the order parameter and therefore suppress the nonspherical $p$-wave pairing $[98,99]$. Furthermore, it is necessary to ensure a high degree of the parallelism of the magnetic field to the plane of the layer, because the presence of even a relatively small perpendicular component would lead to the diamagnetic suppression of superconductivity [96]. Nevertheless, the proposed mechanism is very interesting for the possible realization of superconductivity in very pure heterostructures (see Section [10).

\section{Nontrivial corrections to the Landau Fermi liquid theory in 2D low-density systems}

It is well known that a high temperature of the superconducting transition in cuprate superconductors is connected with very unusual properties of these systems in the normal (nonsuperconducting) state. Among the unconventional properties of the normal 
phase, the small jump in the distribution function of the interacting particles on the Fermi surface and a linear temperature dependence of the resistivity at temperatures much lower than Debye temperature in optimally doped cuprate superconductors are of interest. To explain these facts, Anderson advanced a concept of a Luttinger-type Fermi liquid with a zero jump of the distribution function on the Fermi surface [100]. A similar idea of a marginal Fermi liquid, which is a special case of a Luttinger liquid, was proposed by the authors of [101] based on the analysis of the experimental data.

Later on, Anderson advanced an even more nontrivial idea that not only a high-density strongly interacting 2D Fermi system but even a weakly interacting low-density 2D Fermi gas should also described by a Luttinger Fermi liquid [102] rather than by the Landau Fermi-liquid theory with a finite jump of the distribution function. In Refs [100, 102, Anderson formulated three important points, which led to his doubts regarding the applicability of the standard Galitskii-Bloom Fermi-gas approach [42,46] in the 2D case. These are, firstly, the problem of the finite scattering phase-shift for the particles with almost parallel spins, which leads to the vanishing of the $Z$-factor (Migdal jump) on the Fermi surface; secondly, the problem (connected with the first one) concerning the essential role of the upper Hubbard band in the lattice models already in the case of a low electron density; and, finally, the problem of the possible existence of a strong singularity in the Landau $f$-function for the quasiparticles interaction, which arises in a 2D Fermi gas even in the absence of a lattice.

Many theorists participated in the discussion developed after the publication of Anderson's work; most of them [103 107] supported the Fermi-gas ideology and attempted to prove its consistency in the $2 \mathrm{D}$ case using ladder and parquet approximations in terms of the diagrammatic technique. Anderson continued to insist on his point of view, assuming that the diagrammatic technique is inapplicable to the $2 \mathrm{D}$ systems even at the level of summing up an infinite series of parquet diagrams. In fact, the dispute considered the problem of the choice of a correct ground state, which would allow us to construct a regular procedure of successive approximations in the interaction (or, to be more precise, in its part that was not taken into account in choosing the ground state). According to Anderson's qualitative considerations, the Landau function $f\left(\mathbf{p}, \mathbf{p}^{\prime}\right)$ of the interaction of quasiparticles with almost parallel momenta $\mathbf{p}$ and $\mathbf{p}^{\prime}$ and opposite spins of the colliding particles in the $2 \mathrm{D}$ case, when there is a small deviation from the Fermi surface for $\mathbf{p}$ and $\mathbf{p}^{\prime}$, contains a singular part of the form

$$
f_{\text {sing }}\left(\mathbf{p}, \mathbf{p}^{\prime}\right) \sim \frac{1}{\left|\mathbf{p}-\mathbf{p}^{\prime}\right|}
$$

The existence of such a strong singularity leads to a logarithmic divergence of all Landau harmonics $f_{0}, f_{1}, \ldots$, and, thus, to the complete crush of the Fermi-liquid theory. The accurate calculation of the Landau quasiparticles interaction function $f\left(\mathbf{p}, \mathbf{p}^{\prime}\right)$ carried out in the second order of the perturbation theory in Ref. [106] and, independently, in [107], leads to a significantly weaker singularity in $f$ of the form $\left|\mathbf{p}-\mathbf{p}^{\prime}\right|^{-1 / 2}$, which, in addition to that, exists only in a small window of angles $\phi \propto\left|\mathbf{p}-\mathbf{p}^{\prime}\right|^{3 / 2}$ near the parallel orientation of momenta $\mathbf{p}$ and $\mathbf{p}^{\prime}$. As a result, this singularity leads only to nontrivial temperature corrections to the $f$-function rather than to the destruction of the Fermi-liquid picture as whole.

Concerning the second point of the discussion raised by Anderson, the authors of [108] examined the 2D Hubbard model in the limit of strong coupling $(U \gg W)$ and low electron density $(n \ll 1)$ in the Kanamori $T$-matrix approximation [109]. In the low-energy region $\varepsilon \leq \varepsilon_{F}$, in the framework of this description, the 2D Hubbard model becomes equivalent to a $2 \mathrm{D}$ electron gas with a quadratic spectrum and short-range repulsion (see Section [2). This model can be characterized by the $2 \mathrm{D}$ Bloom gas parameter $f_{0} \approx \frac{1}{\ln \left(1 / n a^{2}\right)}$ [46, which allows to conduct a controlled diagrammatical expansion (here, $n=p_{F}^{2} / 2 \pi$ the electron density in the $2 \mathrm{D}$ case for both spin projections). For the first iteration of the self-consistent $T$-matrix approximation, the authors of [108 found the contribution from the $T$-matrix pole corresponding to the upper Hubbard band. As a result, a dressed one-particle Green's function was obtained with a double-pole structure [108, which resembles the Green function in the Hubbard-I approximation [51]:

$$
\begin{gathered}
G(\omega, \mathbf{k}) \approx \frac{\left(1-n a^{2} / 2\right)}{\omega-\xi_{\mathbf{k}}\left(1-n a^{2} / 2\right)+i o} \\
+\frac{n a^{2} / 2}{\omega-U\left(1-n a^{2} / 2\right)-\xi_{\mathbf{k}} n a^{2} / 2+i o} .
\end{gathered}
$$

where $o$ is the notation for infinitesimally small imaginary part The first term in the right-hand side of (29) corresponds to the contribution from the lower Hubbard band, and the second term corresponds to the contribution from the upper Hubbard band. We note that the second iteration of the self-consistent $T$ matrix approximation does not change the principal 
properties of expression (29). Thus, the presence of the upper Hubbard band leads to nontrivial corrections to the Landau Fermi liquid picture at low electron densities without total destruction of this picture in the $2 \mathrm{D}$ case. More specifically, they produce only small Hartree-Fock corrections to the thermodynamic potential.

We note that all the results concerning superconductivity in the Hubbard model obtained in the singlepole approximation for the one-particle Green function remain valid at $U \gg W$ and low electron density (up to small corrections $\sim W / U$, where $W$ is the bandwidth), when the second pole is taken into account. Thus, this result concerning the two-pole structure of the Green function plays the role of a very interesting bridge connecting the exact results of Galitskii and the Hubbard-I approximation (the Gutzwiller approximation) in the Hubbard model. At the same time, it does not affect the type of pairing or the estimate of the critical temperature at low electron densities.

\section{Superconductivity in the Shubin-Vonsovsky model}

The authors of [110] raised an important problem of the role of full (not reduced) Coulomb interaction in the nonphonon superconductivity mechanisms, which in real metals does not limited to the short-range Hubbard repulsion. The authors of [110] examined the 3D jelly model with realistic values of the electron density, when the Wigner-Seitz correlation radius is not very large $r_{S} \leq 20$,

$$
r_{S}=\frac{1.92}{p_{F} a_{B}},
$$

where $a_{B}=\frac{\varepsilon_{0}}{m e^{2}}$ is the Bohr radius of the electron $(\hbar=1)$. In calculation of the effective interaction, the contributions from the first and second order caused by all diagrams presented in Fig. 2 were taken into account. The authors of [110] noted that the previous studies of Kohn-Luttinger superconductivity were mainly limited to the calculation of only the shortrange Hubbard interaction of electrons $U$, in view of the computational difficulties connected with taking into account the first and higher orders of the Fourier transform of the long-range Coulomb repulsion $V_{\mathbf{q}}$ (depending on the wave vector $\mathbf{q}$ ) in the diagrams. As a result, the strong long-range Coulomb repulsion in the first order of the perturbation theory (the first diagram in Fig. 2) was ignored, and the contribution of the electrons to the effective interaction in the Cooper channel, which was determined only by the last second-order (exchange) diagram in Fig. 2 had an attractive nature and ensured $p$-wave pairing in the $3 \mathrm{D}$ case 38,39 and $d$-wave pairing in the $2 \mathrm{D}$ case [40,67].

In Ref. [110, the long-range Coulomb interaction $V_{\mathbf{q}}$ was chosen in the form of the Fourier transform of the Yukawa potential $V(r)=\frac{e^{2}}{r} \exp (-\kappa r)$, which in the $3 \mathrm{D}$ case takes the standard form

$$
V_{\mathbf{q}}=\frac{4 \pi e^{2}}{q^{2}+\kappa^{2}}
$$

where $\kappa$ is the reciprocal Debye screening length. The authors of 110 concluded, based on the results of calculations, that the low and intermediate values of the Hubbard repulsion $U$ in the presence of the longrange part of Coulomb interaction (31) do not lead to the Cooper instability both in 3D and 2D Fermi systems in the $p$-wave and $d$-wave channels, irrespective of how small the screening length is. The pairing appearing at large orbital momenta $(l \geq 3)$ leads to the almost zero values of the critical temperature at any reasonable value of the Fermi energy. According to the authors of [110, the anomalous pairing caused by strong Coulomb repulsion cannot be measured experimentally in practice, since the corresponding condensation energy (if it exists) is several times lower than the condensation energy caused by the electronphonon interaction.

The growth of interest in the problem of account for the long-range part of Coulomb correlations in the description of the phase diagram of high-temperature superconductors raised the popularity of the extended Hubbard model that includes the interaction between the electrons located on different sites of the crystal lattice (in the Russian literature, this model is often called the Shubin-Vonsovsky model [111-113]).

In the historical aspect, the Shubin-Vonsovsky model, which was formulated almost immediately after the creation of quantum mechanics, is a predecessor of some important models in the theory of strongly correlated electronic systems, in particular, the $s-d(f)$ model and the Hubbard model itself. The Shubin-Vonsovsky model was actively used in studies of polar states in solids [114,115], for describing the metal-insulator transition [116, and also in the study of the influence of the intersite Coulomb repulsion on the effective band structure and superconducting properties of strongly correlated systems [117 119].

In the Wannier representation, the Hamiltonian of the Shubin-Vonsovsky model can be written as 


$$
\begin{aligned}
\hat{H}^{\prime}= & \sum_{f \sigma}(\varepsilon-\mu) c_{f \sigma}^{\dagger} c_{f \sigma}+\sum_{f m \sigma} t_{f m} c_{f \sigma}^{\dagger} c_{m \sigma} \\
& +U \sum_{f} \hat{n}_{f \uparrow} \hat{n}_{f \downarrow}+\frac{1}{2} \sum_{f m \sigma \sigma^{\prime}} V_{f m} \hat{n}_{f \sigma} \hat{n}_{m \sigma^{\prime}},
\end{aligned}
$$

where the last term in the right-hand side corresponds to the energy $V_{f m}$ of the Coulomb interaction of electrons that are located on different sites of the crystalline lattice. The last three terms together in the right-hand side of (32) reflect the fact that the screening radius in the systems in question is equal to several lattice spacings [116]. This determines the efficiency of the Shubin-Vonsovsky model, in which the intersite Coulomb interaction is taken into account within several coordination spheres. In the momentum representation, the Hamiltonian (32) takes the form

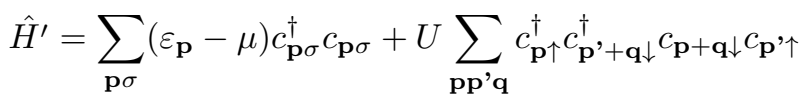

$$
\begin{aligned}
& +\frac{1}{2} \sum_{\mathbf{p} \mathbf{p}^{\prime} \mathbf{q} \sigma \sigma^{\prime}} V_{\mathbf{p}-\mathbf{p}}, c_{\mathbf{p} \sigma}^{\dagger} c_{\mathbf{p}{ }^{\prime}+\mathbf{q} \sigma^{\prime}}^{\dagger} c_{\mathbf{p}+\mathbf{q} \sigma^{\prime}} c_{\mathbf{p}{ }^{\prime} \sigma},
\end{aligned}
$$

where the Fourier transform of the Coulomb interaction between the electrons located on the nearestneighbor sites, $V_{1}$, and on the next-nearest sites, $V_{2}$, of the square lattice in the $2 \mathrm{D}$ case is written as

$$
V_{\mathbf{q}}=2 V_{1}\left(\cos q_{x} a+\cos q_{y} a\right)+4 V_{2} \cos q_{x} a \cos q_{y} a .
$$

The authors of [120] made a contribution to the discussion in 67, 110, by investigating the conditions of the appearance of the Kohn-Luttinger superconducting pairing in the 3D and 2D Shubin-Vonsovsky models with a Coulomb repulsion of the electrons located on neighboring sites $\left(V_{1} \neq 0, V_{2}=0\right)$. As for the interaction, they considered the maximally strong Coulomb repulsion on both the same and neighboring sites: $U \gg V_{1} \gg W$ ( $W$ is the bandwidth; $W=12 t$ for the $3 \mathrm{D}$ cubic lattice and $W=8 t$ for the $2 \mathrm{D}$ square lattice).

On the cubic lattice in the $3 \mathrm{D}$ case, we have the following expressions for the bare interaction of electrons in vacuum in the $s$-wave and $p$-wave channels:

$$
U_{\mathrm{vac}}^{s}=U+6 V+o\left(p^{2} a^{2}\right), \quad U_{\mathrm{vac}}^{p}=2 V \mathbf{p p}^{\prime} a^{2} .
$$

In this case, the $T$-matrices in the appropriate channels in the strong-coupling limit are determined as

$$
T_{s}=\frac{4 \pi}{m} a_{s}, \quad T_{p}=\frac{4 \pi}{m} 2 a_{p} \mathbf{p p}^{\prime} a^{2}
$$

where $a_{s} \sim a$ and $a_{p} \sim a$ for the scattering lengths in the $s$-wave and $p$-wave channels. As a result, the dimensionless gas parameter in the $s$-wave channel takes the form $\lambda_{s}=\lambda=\frac{2 a p_{F}}{\pi}$, just as in the Hubbard model, whereas the bare gas parameter $\lambda_{p}$ in the $p$ wave channel is proportional to $\left(p_{F} a\right)^{3}$, in accordance with the general quantum-mechanical results for slow particles $\left(p_{F} a<1\right)$ in vacuum [121.

Thus, even in the maximally repulsive 3D ShubinVonsovsky model, which is the most unfavorable for the appearance of effective attraction and superconductivity, the normal state in the strong-coupling regime with low electron density is unstable with respect to the triplet $p$-wave pairing. Notably, the effective interaction of electrons at $l=1$ in the substance takes the form [120]

$$
\rho_{3 D} U_{\mathrm{eff}}^{l=1}=\lambda_{p}-\frac{\lambda_{s}^{2}}{13},
$$

where $\rho_{3 D}=m p_{F} /\left(2 \pi^{2}\right)$ is the density of states in the $3 \mathrm{D}$ Fermi gas. As was mentioned above, the contribution from the $p$-wave harmonic of the polarization operator $\Pi_{l=1}$ in substance, $-\lambda_{s}^{2} / 13<0$, favors attraction, and it cannot be compensated by the contribution from the intersite Coulomb repulsion $V_{1}$ in the $p$-wave channel, which is proportional to $\left(p_{F} a\right)^{3}$.

Similarly, in the 2D case, in the regime of strong coupling and low electron density, the dimensionless gas parameter in the $s$-wave channel is $f_{s}=f_{0} \sim$ $\frac{1}{\ln \left(1 / n a^{2}\right)} \sim \frac{1}{\ln \left[1 /\left(p_{F} a\right)^{2}\right]}$, just as in the $2 \mathrm{D}$ Hubbard model, whereas the dimensionless gas parameter in the $p$-wave channel is $f_{p} \sim p_{F}^{2} a^{2}$, again in agreement with the results for slow particles in vacuum. The effective interaction in the $2 \mathrm{D}$ case in substance takes the form 120 .

$$
\rho_{2 D} U_{\mathrm{eff}}^{l=1}=-6.1 f_{s}^{3}+2 p_{F}^{2} a^{2} .
$$

where $\rho_{2 D}=m /(2 \pi)$ is the density of states of a $2 \mathrm{D}$ Fermi gas. Since $f_{s}^{3} \gg p_{F}^{2} a^{2}$ for $p_{F} a \ll 1$, we obtain $U_{\mathrm{eff}}^{l=1} \approx-6.1 f_{s}^{3}$, as in the case $V_{1}=0$ (see Section 8 ).

Thus, the previous results concerning the realization of superconducting $p$-wave pairing in both $3 \mathrm{D}$ and $2 \mathrm{D}$ repulsive Hubbard models at strong coupling $(U \gg W)$ with low electron density remain valid even when we take the strong Coulomb repulsion $V_{1} \gg W$ of electrons at the nearest sites into account in the framework of the Shubin-Vonsovsky model. As a result, the same expressions for the main exponent (which determines the critical temperature of $p$-wave pairing (6) and (11)), are obtained just as in the absence of the intersite Coulomb repulsion $\left(V_{1}=0\right)$ in both three-dimensional and twodimensional cases. Account for $V_{1}$ changes only the preexponential factor [48, which means that the superconducting $p$-wave pairing can be developed in Fermi systems with pure repulsion [120] (in the absence of electron-phonon interaction) even in the presence of the long-range Coulomb repulsion. 
The authors of 122] carried out a similar analysis for the extended Hubbard model in the Born weakcoupling approximation and came to the same conclusions as the authors of [120]. Moreover, it was noted in [122] that in the weak-coupling regime $W>U>V$, the effect of the long-range Coulomb interactions is also suppressed, and does not impair the conditions for the development of Cooper instability. This is explained by the fact that the long-range interactions in the lattice models usually contribute only to some specific channels of pairing and do not affect the other channels. At the same time, the polarization contributions that are described by the diagrams shown in Fig. 2 have components in all the channels and usually more than one of them favors attraction. In this situation, it turns out that the long-range interactions either do not influence the principal components of the effective interaction which lead to the pairing or suppress the main components but do not affect the secondary ones [see the discussion after expression (49)] ].

In this connection, a phase diagram was constructed in Ref. 122] based on the extended Hubbard model in the framework of the Kohn-Luttinger mechanism, which clearly reflects the result of the competition of the superconducting phases with different types of the symmetry of order parameter. In the calculations of the effective coupling constant, an expression for the renormalized scattering amplitude in the Cooper channel was used in the form

$$
U_{\text {eff }}(\mathbf{p}, \mathbf{q})=U+V_{\mathbf{p}-\mathbf{q}}+U^{2} \Pi(\mathbf{p}+\mathbf{q}),
$$

where $V_{\mathbf{p}-\mathbf{q}}$ is the Fourier transform of the intersite Coulomb repulsion of electrons, Eqn (34), and $\Pi(\mathbf{p}+\mathbf{q})$ is the Lindhard function (4) . Thus, the intersite Coulomb interaction $V$ was taken into account only in the first order of the perturbation theory, and the polarization contributions were determined only by the term of the order of $U^{2}$. It was shown in 122 that although the long-range interactions have a tendency to suppress the anomalous pairing in some channels, the Kohn-Luttinger superconductivity survives in the entire region of electron concentrations $0<n<1$ and for all relations of the model parameters.

It was noted in Refs [123, 124 that the effective interaction $U_{\text {eff }}(\mathbf{q})$ is characterized by a dependence that is quadratic in the quasimomentum only in the region of $\mathbf{q a} \ll 1$. Outside this region, the dependence of $V_{\mathbf{q}}$ on the momentum is determined by periodic functions. As a result, the behavior of $U_{\text {eff }}(\mathbf{q})$ is modified significantly in comparison with the behavior of the momentum dependence of the Fourier transform of the Yukawa potential. These factors substantially affect the conditions of the realization of Cooper instability at large electron densities, when the Fermi surfaces do not have the spherical symmetry. Therefore, it can be expected that the conditions for the realization of superconducting pairing in the framework of the Kohn-Luttinger mechanism are determined not only by the dynamic effects caused by the Coulomb interactions but also by the effects related to the Brillouin zone.

The authors of [123, 124] discussed the influence of the Coulomb interaction of electrons located in the first and second coordination spheres on the development of Cooper instability in the Born weak-coupling approximation, $W>U>V$. Accordingly, they used the effective interaction $U_{\text {eff }}(\mathbf{p}, \mathbf{k})$, which is determined in the graphic form by the sum of five diagrams (see Fig. 2) and for the Shubin-Vonsovsky model has the following analytic form

$$
\begin{aligned}
U_{\mathrm{eff}}(\mathbf{p}, \mathbf{k}) & =U+V_{\mathbf{p}-\mathbf{k}}+\delta U(\mathbf{p}, \mathbf{k}) \\
\delta U(\mathbf{p}, \mathbf{k}) & =\frac{1}{N} \sum_{\mathbf{p}_{1}}\left(U+V_{\mathbf{p}-\mathbf{k}}\right)\left(2 V_{\mathbf{p}-\mathbf{k}}-V_{\mathbf{p}_{1}+\mathbf{p}}-V_{\mathbf{p}_{1}-\mathbf{k}}\right) \\
& \times \frac{n_{F}\left(\varepsilon_{\mathbf{p}_{1}}\right)-n_{F}\left(\varepsilon_{\mathbf{p}_{1}+\mathbf{p}-\mathbf{k}}\right)}{\varepsilon_{\mathbf{p}_{1}}-\varepsilon_{\mathbf{p}_{1}+\mathbf{p}-\mathbf{k}}}+ \\
& +\frac{1}{N} \sum_{\mathbf{p}_{1}}\left(U+V_{\mathbf{p}_{1}-\mathbf{p}}\right)\left(U+V_{\mathbf{p}_{1}-\mathbf{k}}\right) \\
& \times \frac{n_{F}\left(\varepsilon_{\mathbf{p}_{1}}\right)-n_{F}\left(\varepsilon_{\mathbf{p}_{1}-\mathbf{p}-\mathbf{k}}\right)}{\varepsilon_{\mathbf{p}_{1}-\mathbf{p}-\mathbf{k}}-\varepsilon_{\mathbf{p}_{1}}} .
\end{aligned}
$$

The presence of the renormalized expression for the effective interaction allows us to analyze the conditions for the realization of the Cooper instability. Taking into account the fact that the leading contribution to the total scattering amplitude of two electrons with opposite momenta and spin projections $\Gamma$ (the total amplitude in the Cooper channel) is determined by electron scattering near the Fermi surface, the dependence of $\Gamma$ on the Matsubara frequency can be neglected in the Bethe-Salpeter integral equation. As a result, this equation is simplified taking the form

$\Gamma(\mathbf{p} \mid \mathbf{k})=U_{\mathrm{eff}}(\mathbf{p}, \mathbf{k})-\frac{1}{N} \sum_{\mathbf{q}} U_{\mathrm{eff}}(\mathbf{p}, \mathbf{q}) L\left(\xi_{\mathbf{q}}\right) \Gamma(\mathbf{q} \mid \mathbf{k})$,

where $L\left(\xi_{\mathbf{q}}\right)=\tanh \left(\xi_{\mathbf{q}} / 2 T\right) / 2 \xi_{\mathbf{q}}$ is the standard expression for the kernel of the Cooper loop.

It is known 29] that the appearance of Cooper instability can be extracted from an analysis of the homogeneous part of Eqn (42). In this case, the dependence of $\Gamma$ on the momentum $\mathbf{k}$ is factorized and can be neglected. As a result, we proceed from (42) an integral Gor'kov equation for the superconducting gap $\Delta(\mathbf{p})$. Passing to the integration with respect to 
a)

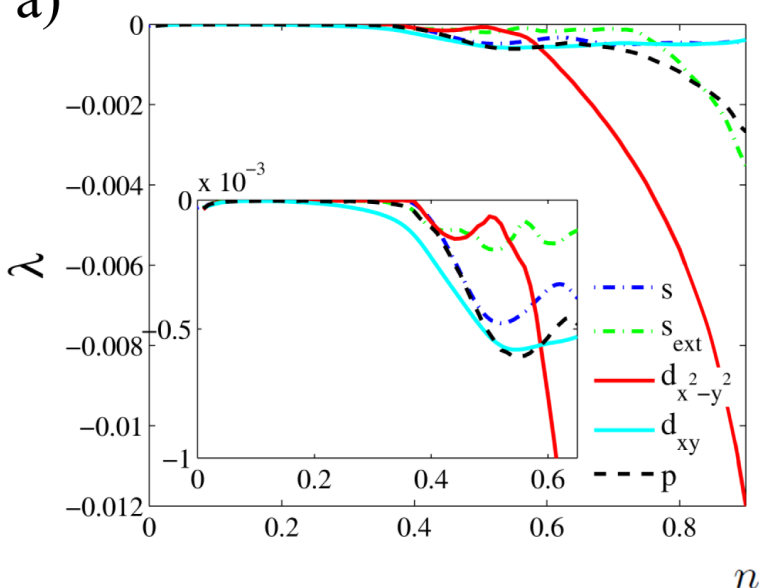

b)

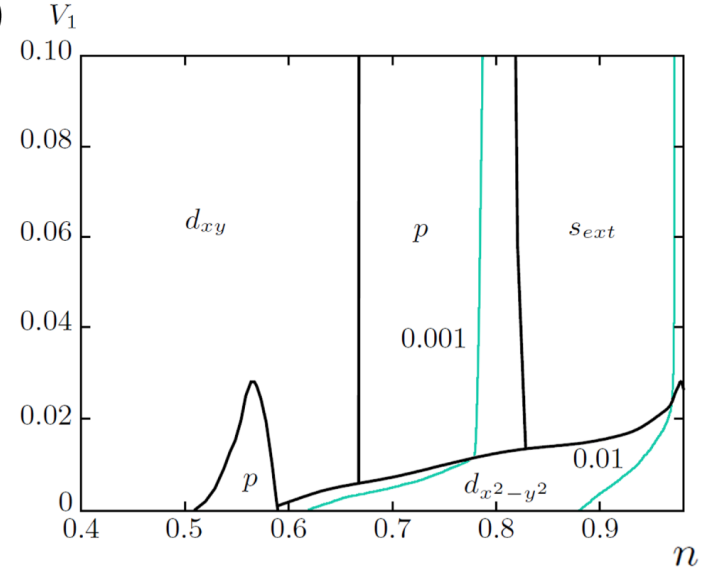

Fig. 5. (a) Dependencies of $\lambda$ on the electron concentration $n$ at $t_{2}=t_{3}=0, U=\left|t_{1}\right|$ and $V_{1}=V_{2}=0$; and (b) the " $n-V_{1}$ " phase diagram of the ShubinVonsovsky model on a square lattice at $t_{2}=t_{3}=$ $0, U=\left|t_{1}\right|$ and $V_{2} / V_{1}=0$. The intersite Coulomb repulsion is taken into account only in the first order of the perturbation theory [123, 124]. For all points that belong to one thin line in (b), the indicated value of $|\lambda|$ is constant.

the constant-energy contours (in the 2D case), we obtain that the possibility of the Cooper pairing is determined by the characteristics of the energy spectrum in the vicinity of the Fermi level and by the effective interaction $U_{\text {eff }}(\mathbf{p}, \mathbf{k})$ of the electrons that are located near the Fermi surface $\varepsilon_{\mathbf{q}}=\mu[59,60]$. As a result, the analysis of the Cooper instability reduces to the solution of the eigenvalue problem

$$
\frac{1}{(2 \pi)^{2}} \oint_{\varepsilon_{\mathbf{q}}=\mu} \frac{d \hat{\mathbf{q}}}{v_{F}(\hat{\mathbf{q}})} U_{\mathrm{eff}}(\hat{\mathbf{p}}, \hat{\mathbf{q}}) \Delta(\hat{\mathbf{q}})=\lambda \Delta(\hat{\mathbf{p}}),
$$

in which the superconducting order parameter $\Delta(\hat{\mathbf{q}})$ plays the role of an eigenvector, and we have the eigenvalues of $\lambda^{-1} \simeq \ln \left(T_{c} / W\right)$. In this case, the quasimo- menta $\hat{\mathbf{p}}$ and $\hat{\mathbf{q}}$ lie on the Fermi surface, and $v_{F}(\hat{\mathbf{q}})$ is the Fermi velocity. The feasible solutions of Eqn (43) with $\lambda<0$ are determined not only by the effective interaction $U_{\text {eff }}(\mathbf{p}, \mathbf{q})$ but also by the shape of isoenergetic contours. As far as the concrete structure of these contours is closely connected with the energy spectrum, it is obvious that if we are not limited to the nearest-neighbor approximation and take into account the distant hoppings, we can substantially influence the conditions for the realization of the Cooper instability and significantly modify the structure of the phase diagram of the superconducting state.

To solve Eqn (43), we represent its kernel in the form of a superposition of the functions which belong to one of the irreducible representations of the symmetry group $C_{4 v}$ of the square lattice. As it is well known, this group has five irreducible representations [121, and for each of them Eqn (43) has its solution with an appropriate effective coupling constant $\lambda$. Subsequently, the following classification is used for the symmetry of the order parameter: the representation $A_{1}$ corresponds to $s$-wave type symmetry; $A_{2}$ to the extended $s$-wave type symmetry $\left(s_{\text {ext }}\right) ; B_{1}$ to the $d_{x y}$-wave type symmetry; $B_{2}$ to the $d_{x^{2}-y^{2}}$-wave type symmetry; and $E$ to the $p$-wave type symmetry.

For the irreducible representations $\gamma=$ $A_{1}, A_{2}, B_{1}, B_{2}, E$, the solution of Eqn (43) is searched in the form

$$
\Delta^{(\gamma)}(\phi)=\sum_{m} \Delta_{m}^{(\gamma)} g_{m}^{(\gamma)}(\phi)
$$

where $m$ is the number of the basis function of the representation $\gamma$, and $\phi$ is the angle that characterizes the direction of the momentum $\hat{\mathbf{p}}$ with respect to the axis $p_{x}$. The explicit form of $g_{m}^{(\gamma)}(\phi)$ is determined by the following expressions:

$$
\begin{aligned}
& A_{1} \rightarrow g_{m}^{(s)}(\phi)=\frac{1}{\sqrt{\left(1+\delta_{m 0}\right) \pi}} \cos 4 m \phi, \quad m \in[0, \infty) \\
& A_{2} \rightarrow g_{m}^{\left(s_{e x t}\right)}(\phi)=\frac{1}{\sqrt{\pi}} \sin 4(m+1) \phi, \\
& B_{1} \rightarrow g_{m}^{\left(d_{x y}\right)}(\phi)=\frac{1}{\sqrt{\pi}} \sin (4 m+2) \phi, \\
& B_{2} \rightarrow g_{m}^{\left(d_{\left.x^{2}-y^{2}\right)}(\phi)\right.}=\frac{1}{\sqrt{\pi}} \cos (4 m+2) \phi, \\
& E \rightarrow g_{m}^{(p)}(\phi)=\frac{1}{\sqrt{\pi}}(A \sin (2 m+1) \phi \\
& \quad+B \cos (2 m+1) \phi) .
\end{aligned}
$$

The basis functions satisfy the orthonormalization conditions

$$
\int_{0}^{2 \pi} d \phi g_{m}^{(\gamma)}(\phi) g_{n}^{(\beta)}(\phi)=\delta_{\gamma \beta} \delta_{m n}
$$



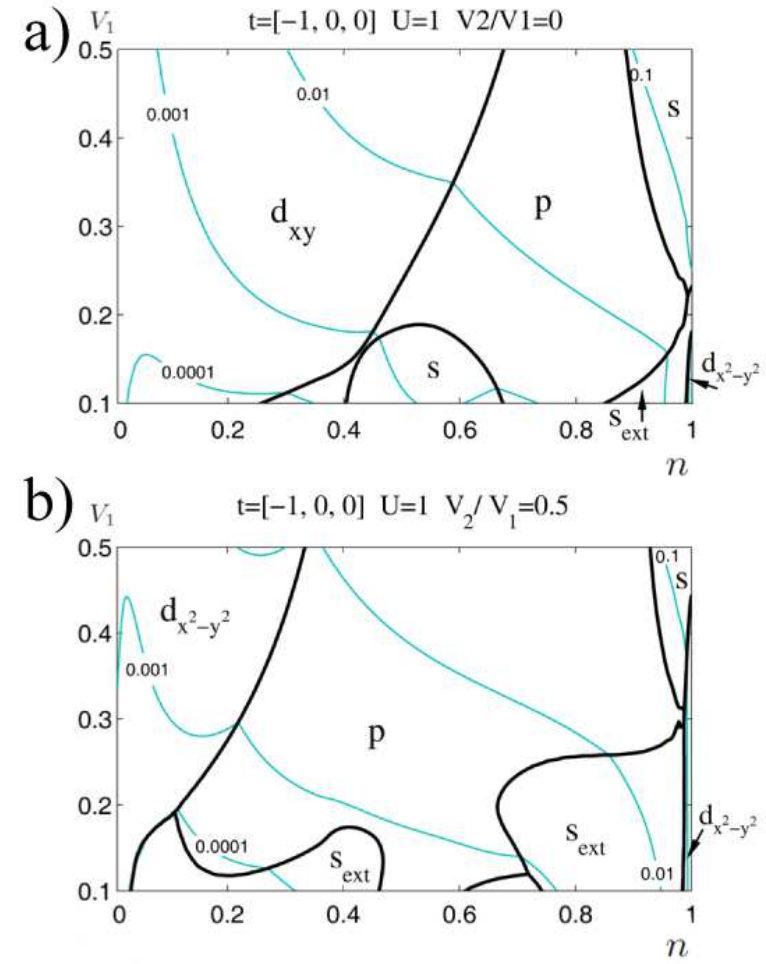

Fig. 6. The " $n-V_{1}$ " phase diagram of the ShubinVonsovsky model on a square lattice constructed taking into account the contributions of the second order in $V$ at the parameters $t_{2}=t_{3}=0, U=\left|t_{1}\right|$ and the ratios (a) $V_{2} / V_{1}=0$ and (b) $V_{2} / V_{1}=0.5$. The thin lines correspond to the lines of constant $|\lambda|$ [124].

Substituting (44) in Eqn (43), integrating with respect to the angles, and using the orthonormalization condition for the functions $g_{m}^{(\gamma)}(\phi)$, we obtain

$$
\sum_{n} \Lambda_{m n}^{(\gamma)} \Delta_{n}^{(\gamma)}=\lambda_{\gamma} \Delta_{m}^{(\gamma)}
$$

where

$$
\begin{aligned}
\Lambda_{m n}^{(\gamma)} & =\frac{1}{(2 \pi)^{2}} \oint_{0}^{2 \pi} d \phi_{\mathbf{p}} \oint_{0}^{2 \pi} d \phi_{\mathbf{q}} \frac{d \hat{\mathbf{q}}}{d \phi_{\mathbf{q}} v_{F}(\hat{\mathbf{q}})} U_{\mathrm{eff}}(\hat{\mathbf{p}}, \hat{\mathbf{q}}) \\
& \times g_{m}^{(\gamma)}\left(\phi_{\mathbf{p}}\right) g_{n}^{(\gamma)}\left(\phi_{\mathbf{q}}\right) .
\end{aligned}
$$

Since $T_{c} \sim W \exp (1 / \lambda)$, each negative eigenvalue $\lambda_{\gamma}$ corresponds to a superconducting phase with the $\gamma$ wave symmetry of the order parameter. The expansion of the order parameter $\Delta^{(\gamma)}(\phi)$ in the basis functions includes many harmonics in general, but the leading contribution is determined by only several first terms. The largest value of the critical temperature is associated with the largest value of $\left|\lambda_{\gamma}\right|$.

If the intersite Coulomb interaction is taken into account only for electrons that are located on the nearest sites $\left(V_{1} \neq 0, V_{2}=0\right.$ in (34) $)$ and the excitation spectrum is described by only one hopping parameter $\left(t_{1} \neq 0, t_{2}=t_{3}=0\right)$, then the phase diagram of the superconducting state at $U=\left|t_{1}\right|$ (Fig. [5) contains five regions. Figure $5 \mathrm{~b}$ displays the dependence of the effective coupling constants $\lambda$ for different types of the symmetry of the superconducting order parameter on the electron density $n$ obtained at $V_{1}=V_{2}=0$. Based on the $\lambda(n)$ dependencies, a phase diagram for different values of the intersite Coulomb repulsion $V_{1}$, which reflects the competition between the superconducting phases with the different symmetry types of the order parameter can be constructed (Fig. 5b). The case depicted in Fig. 5 a corresponds to the abscissa axis in Fig. 5b. To construct this phase diagram, Eqn (39) for the effective interaction of electrons in the Cooper channel was used, which takes contributions of only the first order in $V$ into account and ignores the contributions proportional to $U V$ and $V^{2}$. The region of the phase diagram that lie on the abscissa axis $\left(V_{1}=0\right)$ are in a good agreement with the phase diagrams regions obtained in Refs [63, 125]. In the region of low and intermediate densities of electrons, $n=0-0.52$, in the two first orders of the perturbation theory, superconductivity with the $d_{x y}$ symmetry type of the order parameter [40,57] is realized; in the range of $n=0.52-0.58$, the ground state corresponds to the phase with $p$-wave pairing, but in this case $\left|\lambda_{p}\right|$ insignificantly exceeds $\left|\lambda_{d_{x y}}\right|$ (see the inset in Fig. $5 \mathrm{a}$ ). We note that according to the calculations of the authors of [122], $d_{x y}$-wave pairing is realized instead of $p$-wave pairing in this interval of electron densities. At $n>0.58$, the $d_{x^{2}-y^{2}}$-wave type of superconductivity appears, which is relevant for cuprate superconductors.

Note that with an account of the Coulomb repulsion $V_{1}$ on neighboring sites in the first order of the perturbation theory, the absolute value of $\lambda$ decreases for all types of symmetry. In this case, the superconducting $d_{x^{2}-y^{2}}$-wave phase is suppressed most strongly and, with increasing $V_{1}$, the phases with the order parameters that have different symmetry types are realized at these concentrations.

The first order of the perturbation theory in the intersite Coulomb repulsion always has a tendency to suppress the superconducting pairing. Hence, the possibility of realization of the Cooper instability based on the Kohn-Luttinger mechanism is connected with the appearance in the second order of the perturbation theory of the attractive and sufficiently strong contributions to the effective interaction matrix (41). Thus, in order to take into account the polarization effects for the intersite Coulomb interaction, we should use the full expression (40) for $U_{\text {eff }}(\mathbf{p}, \mathbf{q})$ but not reduced one (39). In this case, the polarization effects proportional to $U V$ and $V^{2}$, even at small values of 


$$
\mathrm{t}_{1}=-1 \quad \mathrm{t}_{2}=0 \quad \mathrm{t}_{3}=0 \quad \mathrm{U}=1 \quad \mathrm{~V}_{1}=0.5 \quad \mathrm{~V}_{2}=0 \quad \mathrm{n}=0.95 \quad \mathrm{~s}-\mathrm{type}
$$

a)

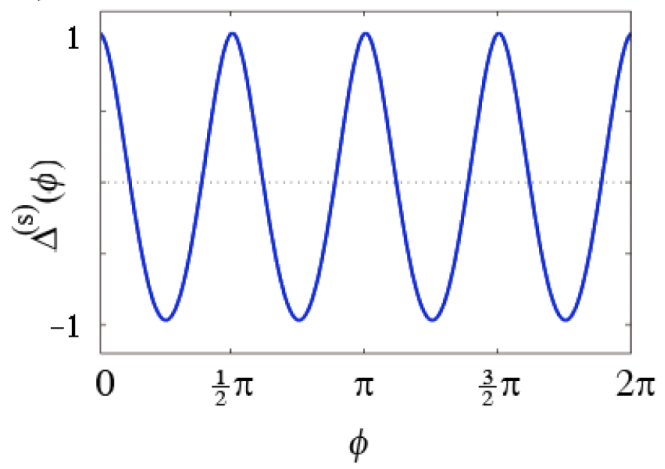

b)

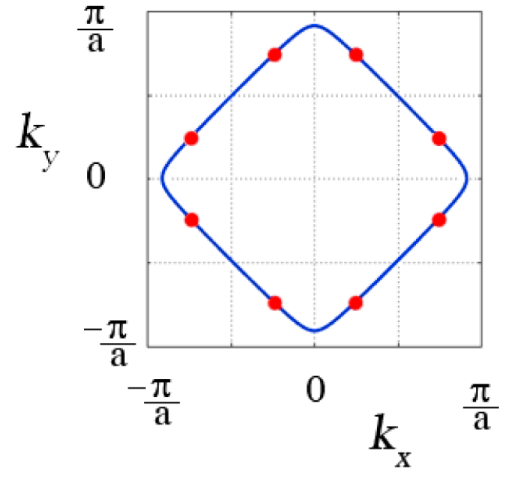

Fig. 7. (a) Angular dependence of the superconducting order parameter $\Delta^{(s)}(\phi)$ and (b) the arrangement of the nodal points (zeroes of the gap $\Delta^{(s)}(\phi)$ ) on the Fermi contour calculated at the parameters $t_{2}=t_{3}=0, U=$ $\left|t_{1}\right|, V_{1}=0.5\left|t_{1}\right|, V_{2}=0$, and $n=0.95$ [124].

$V_{1}$, substantially change and complicate the structure of the phase diagram (Fig. 6a). With an increase of the intersite Coulomb interaction parameter $V_{1}$, an increase of $|\lambda|$ occurs for $T_{c} \sim W \exp (-1 /|\lambda|)$. In this case, only three phases are stabilized, which correspond to the $d_{x y^{-}}, p$-, and $s$-wave symmetry types of the superconducting order parameter.

We note that in the region of high electron concentrations and at $0.25<V_{1} /\left|t_{1}\right|<0.5$, the KohnLuttinger polarization effects lead to the appearance of a superconducting $s$-wave pairing. This qualitative effect clearly demonstrates the importance of the second-order processes in calculating the effective interaction of electrons in the Cooper channel and in constructing the phase diagram presented in Fig. 6. A quantitative comparison of the different partial contributions to the total effective interaction showed that the realization of $s$-wave pairing was due to the polarization contributions proportional to $V^{2}$. In this case, the leading contribution is determined by the angular harmonic $g_{1}^{(s)}(\phi)=\frac{1}{\sqrt{\pi}} \cos 4 \phi$ rather than by the constant (as in the case of the usual $s$-wave pairing in the isotropic situation and in the absence of a lattice).

The above-mentioned scenario of the realization of superconducting $s$-wave pairing due to the higher angular harmonics correlates well with the experimental data recently obtained in Ref. [126, which presents the results of the experimental studies of a superconductor based on the iron arsenide $\mathrm{KFe}_{2} \mathrm{As}_{2}$ by angleresolved photoemission spectroscopy (ARPES). These studies showed that this compound is a nodal superconductor (containing zeroes of the gaps) with an $s$ - wave type symmetry of the order parameter, which has eight points at which the gap vanishes.

Figure 7 a shows the angular dependence of the superconducting order parameter

$$
\begin{aligned}
\Delta^{(s)}(\phi) & =\frac{\Delta_{0}^{(s)}}{\sqrt{2}}+\Delta_{1}^{(s)} \cos 4 \phi+\Delta_{2}^{(s)} \cos 8 \phi \\
& +\Delta_{3}^{(s)} \cos 12 \phi+\Delta_{4}^{(s)} \cos 16 \phi
\end{aligned}
$$

calculated in Ref. 124 for the point of the phase diagram at which the $s$-wave pairing is realized at large electron densities. This dependence demonstrates the presence of eight nodal points at which the gap vanishes. Their arrangement on the Fermi contour (Fig. 7b) according to the results of calculations 124 is in a qualitative agreement with the experimental picture presented in [126].

A similar scenario for the realization of the superconductivity is also observed in the $p$-wave channel. Here, the pairing obtained by taking into account the second order of perturbation theory in the Coulomb interaction is suppressed by the bare repulsion only for the first harmonic $g_{0}^{(p)}(\phi)=\frac{1}{\sqrt{\pi}}(A \sin \phi+B \cos \phi)$. In this case, the leading contribution to $\Delta^{(p)}(\hat{\mathbf{p}})$ comes from the next $p$-wave pairing harmonic on the lattice, $g_{1}^{(p)}(\phi)=\frac{1}{\sqrt{\pi}}(A \sin (3 \phi)+B \cos (3 \phi))$.

The authors of 124 have also analyzed the influence of the Coulomb repulsion between next-tonearest neighbors $\left(V_{2} \neq 0\right)$ and distant electron hoppings $\left(t_{2} \neq 0, t_{3} \neq 0\right)$ on the phase diagram of the superconducting state of the Shubin-Vonsovsky model. Figure 8 shows a modification of the phase diagram 
of the Shubin-Vonsovsky model that takes place with an increase in the Hubbard repulsion parameter. It can be seen that in the region of low electron densities and in the region of densities close to the Van Hove singularity, we get a superconducting phase with a

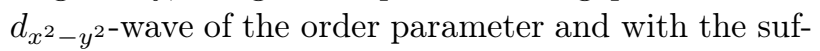
ficiently large values of $|\lambda| \sim 0.1-0.2$. This result can be interesting for the possibility of the implementation of the Kohn-Luttinger mechanism to cuprate superconductors. Note that at $|\lambda| \sim 0.2$ the critical temperatures of the superconducting transition can reach values $T_{c} \sim 100 \mathrm{~K}$ which are quite reasonable for the cuprates.

\section{Superconductivity in the 2D $t-J$ model}

After Anderson advanced an idea 44 that the electronic properties of cuprate superconductors can be described by the Hubbard model in the strongcoupling limit $U \gg W$, the so-called $t-J$ model acquired great popularity among the researchers (see reviews [127-130]). This model was initially derived by a canonical transformation from the Hubbard model near the half-filling, $n \rightarrow 1$, in the limit $t / U<<1$ [131, 132]. Later on, for cuprates, a generalized $t-J$ model was suggested [133, 134, The Hamiltonian of the generalized 2D $t-J$ model with a weakened constraint and an arbitrary ratio $J / t$ derived from the three-band Emery model [135, 136] is written as [133, 134, 137]

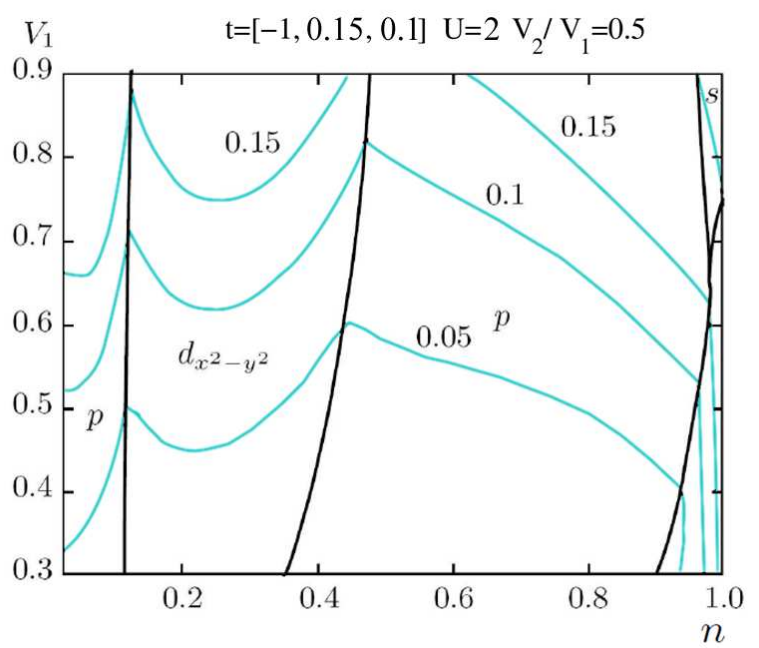

Fig. 8. The " $n-V_{1}$ " phase diagram of the ShubinVonsovsky model on a square lattice, calculated at the parameters $t_{2}=0.15\left|t_{1}\right|, t_{3}=0.1\left|t_{1}\right|, \quad U=$ $2\left|t_{1}\right|, V_{2} / V_{1}=0.5$. The thin lines are lines of constant $|\lambda|[124$.

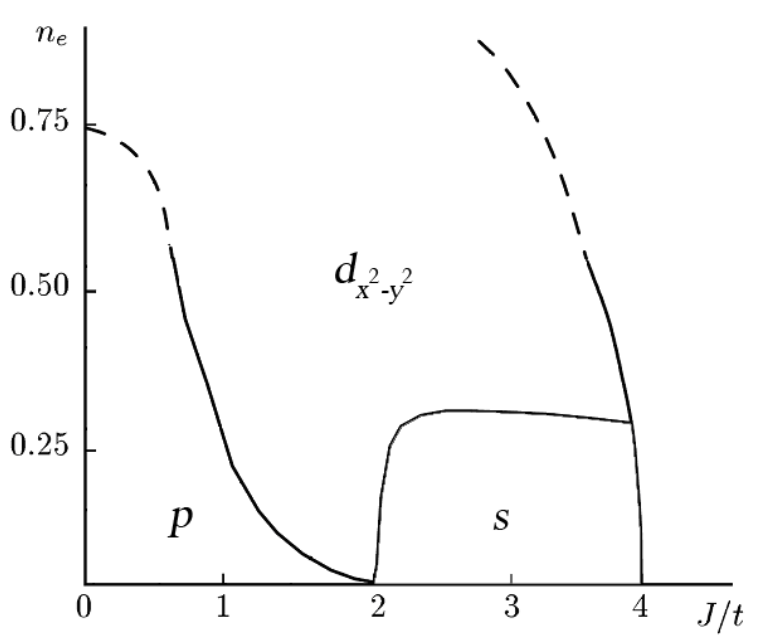

Fig. 9. Phase diagram of the superconducting state of the $2 \mathrm{D} t-J$ model at small and intermediate electron densities [96, 137.

$$
\begin{aligned}
\hat{H}= & \sum_{f \sigma}(\varepsilon-\mu) c_{f \sigma}^{\dagger} c_{f \sigma}+t \sum_{\langle f m\rangle \sigma} c_{f \sigma}^{\dagger} c_{m \sigma} \\
& +U \sum_{f} \hat{n}_{f \uparrow} \hat{n}_{f \downarrow}+J \sum_{\langle f m\rangle}\left(\mathbf{S}_{f} \mathbf{S}_{m}-\frac{\hat{n}_{f} \hat{n}_{m}}{4}\right) .
\end{aligned}
$$

In fact, this is a model with a strong Hubbard repulsion between electrons on one site and weak antiferromagnetic attraction $J>0$ on neighboring sites. The hierarchy of the parameters of the model is $U \gg$ $\{J, t\}$. The phase diagram of the $t-J$ model constructed in [137] is presented in Fig. 9]

For the parameters that are realistic for optimally doped cuprate superconductors, $J / t \sim 0.5$ and $n=2 \varepsilon_{F} / W=0.85$, the critical temperature of the superconducting transition has been estimated as

$$
T_{c}^{d_{x^{2}-y^{2}}} \sim \varepsilon_{F} \exp \left(-\frac{\pi t}{2 J n^{2}}\right) \sim 10^{2} \mathrm{~K} .
$$

We note that a similar estimate for the critical temperature of the $d_{x^{2}-y^{2}}$-wave pairing has been obtained in the framework of a more rigorous spin-polaron theory in 138, 139 with the use of the Hubbard operators 140 .

The authors of [138, 139] also used the generalized $t-J$ model derived from the Emery model in the limit of a small number of holes by constructing the Zhang-Rice singlets 141] at $J<t$ and neglecting the Coulomb repulsion between the charge carriers from the energy bands of copper and oxygen $\left(V_{p d}=0\right)$. In this approximation, the weakened constraint is also not very important, and we can neglect the kinematic interaction 142, 143].

We note that a very interesting physics appears in the so-called 'difficult' comer of the phase diagram of 
the generalized $t-J$ model, namely, in the case of small $J(J / t<<1)$ and low doping $\delta=\left(1-n_{e}\right)<<1$ (this region is frequently called the pseudogap). For this part of the phase diagram, in accordance with the ideas of Laughlin [144,145] on the spin-charge confinement of spin and charge in two-dimensional and threedimensional strongly correlated electron systems (see also [146, 147]), a concept of a strongly interacting Fermi-Bose mixture of spinons and holons has been proposed. The spinons and holons in the mixture form the composite holes in the confinement potential of an antiferromagnetic (AFM) string [131, 148]). In this case, according to the assumption made in [96, 147], the phase diagram of cuprate superconductors in the region of low doping can be considered in the framework of the scenario of the BCS-BEC crossover between the local and extended pairs for pairing of two composite holes (two spin polarons or two AFM strings) in the $d_{x^{2}-y^{2}}$-wave channel. Certainly, the transition from the region of optimal doping with a large Fermi surface and extended Cooper pairs to the region of low doping with local pairs and hole-type pockets (small Fermi surface) can be realized in a very nontrivial way and can contain a singularity in the middle, such as a quantum critical point (QCP) (see, e.g., 149 151]), or even certain intermediate phases.

Returning to the region of the extended Cooper pairing and optimal doping $n_{e} \geq 0.85$, we emphasize that the development and utilization of the KohnLuttinger ideas for the strong-coupling regime would be one of the most challenging directions in the modern theory of superconductivity in strongly correlated systems. However, the solution of this problem requires an account of the strong on-site correlations in all the orders of perturbation theory. Moreover, the intersite correlations must be described taking into account at least the second-order contributions. One of the possibilities to develop the theory in this direction is to use the atomic representation [140, and the diagram technique for the Hubbard operators [152,153]. The relevant models that can be used for the investigation of the Kohn-Luttinger renormalization are the generalized $t-J-V$ model [154 157] and the $t-J^{*}-V$ model (which takes three-center interactions into account), whose important role in describing the superconducting state was studied in [158 165]. These models, which can be derived from the ShubinVonsovsky model in a certain range of parameters, effectively represent its low-energy versions.

Concluding this section, we note that ultracold quantum gases in optical lattices also provide an excellent experimental opportunity to simulate strongly correlated systems on a lattice, in particular, to study the phase diagram of the $t-J$ model and even the structure of the AFM string and spin polarons in a situation with well-controlled and easily tunable parameters $t, J$, and $n_{e}$ [166.

\section{Superconductivity in an idealized graphene monolayer}

Nowadays, the popularity of the Kohn-Luttinger mechanism continues to grow due to the possibility of its utilization in other physical systems. For example, the conditions of its appearance in topological superfluid liquids [167], as well as in the idealized monolayer and bilayer of graphene (where the effect of impurities and the van der Waals potential of the substrate are ignored) are being discussed actively.

At present, graphene is of significant interest from both the fundamental and applied points of view because of its unconventional transport, pseudo-relativistic, and quantum-electrodynamic properties [23, 24, 26]. These properties of graphene are caused, first of all, by its unique gapless energy band structure with the cone-shaped valence and conduction bands (Fig. 10), touching each other at the corners of the first Brillouin zone at the Dirac points [25]. It has been established that near the Dirac points, the electrons propagating in graphene are similar to massless fermions with linear dispersion [168, minimal conductivity at vanishing concentration of carriers [168, 169], high mobility [170 172, Klein tunneling [173, 174], oscillating motion (Zitterbewegung) [175, 176], universal absorption of light [177, and many other properties that have no analogs in other physical systems.

Placed in contact with superconductors, graphene manifests unconventional superconducting properties [178]. The authors of [179] experimentally studied the Josephson effect 180, in mesoscopic junctions consisting of a short sample of graphene monolayer placed between two closely spaced superconducting electrodes. By cooling this device below the critical temperature of the electrodes $\left(T_{c} \approx 1.3 \mathrm{~K}\right)$, the authors of [179] observed a supercurrent in the graphene monolayer (a similar result was obtained in [181]). By changing the voltage of the electric field at the gate electrode, the researchers could shift the Fermi level from the valence band to the conduction band and thus control the density of charge carriers in the graphene monolayer. Irrespective of the position of the Fermi level in the system, a Josephson current was observed, which indicates that this device works as a bipolar supercurrent transistor. Namely, the supercurrent was transferred by $p$-wave type Cooper pairs when the Fermi level was located in the valence band, 

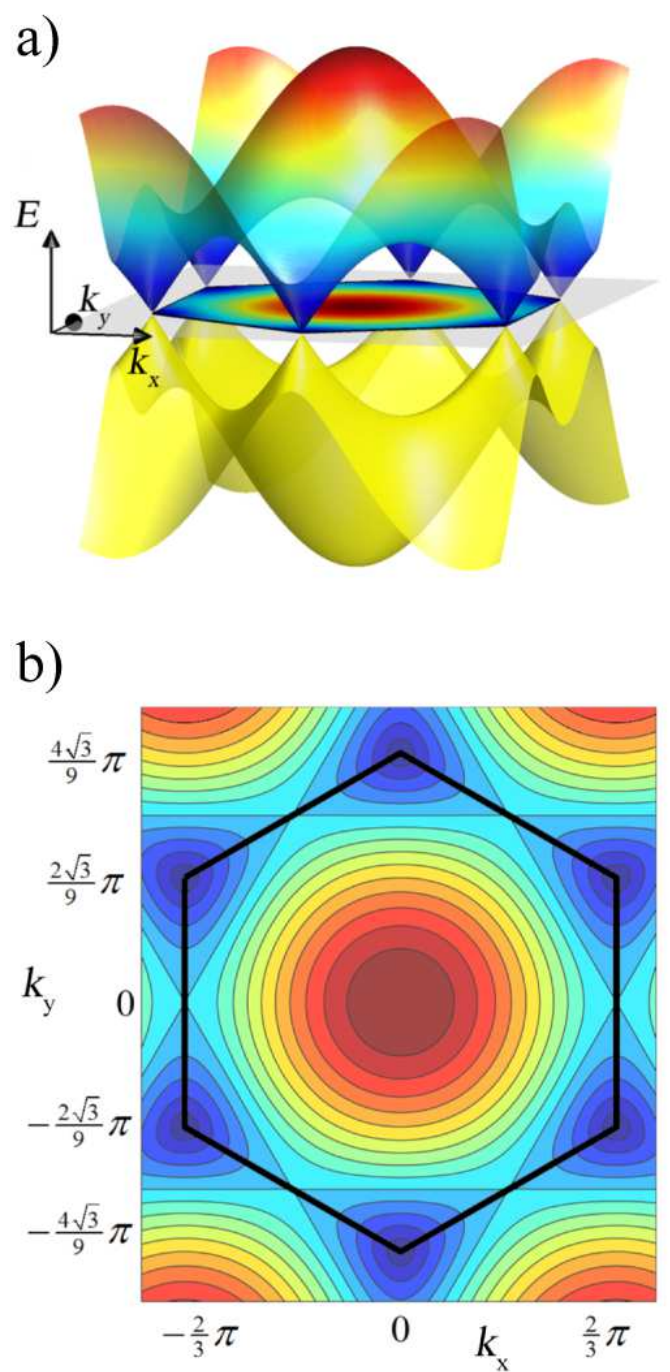

Fig. 10. (a) Energy structure of the graphene monolayer and (b) the energy contours around saddle points in the conduction band of graphene, obtained in the tight-binding model in the nearest-neighbors approximation.

and by electron Cooper pairs when the Fermi level was located in the conduction band. More important is the fact that the supercurrent could flow in the graphene monolayer even when the Fermi level was located precisely at the Dirac point, i.e., at the zero density of carriers. This behavior was explained within the theory of ballistic transport from graphene to the Josephson junctions [182]; however, later experiments [183 185] have demonstrated that the transport in the superconductor-graphene-superconductor junctions is, rather, of a diffusive nature.

Although so far no confirmation has been found that the Cooper instability can be developed in graphene itself (possibly because of permanently present structural disorder), the results of the above- mentioned experiments indicate that the Cooper pairs can propagate in graphene coherently. In this connection, a question arises as to whether it is possible to modify graphene structurally (for example, by introducing twinning planes and grain boundaries) or chemically, such that it would become a magnet [186] or even a true superconductor 187.

The theoretical analysis in [188 has shown that the model with a conical dispersion requires a minimal strength of pairing interaction for the development of Cooper instability in the undoped system. Furthermore, several attempts were undertaken to analyze the possibility of realization of the superconducting state in doped graphene monolayer. In [189, the role of topological defects in the realization of Cooper pairing in this material was studied. The superconducting phase was also investigated and the symmetry of the order parameter was determined on a hexagonal lattice of graphene. In the case of the attractive Hubbard interaction $U$ between electrons, as it was shown in 190, the usual singlet pairing with an $s$-wave type symmetry of the order parameter is realized. In [191], a phase diagram of the superconducting state was constructed (in the mean-field approximation) for a graphene monolayer in the extended Hubbard model with attraction, and the plasmon mechanism of superconductivity was investigated, which led to low critical temperatures in the $s$-wave channel at realistic values of the electron concentration. Furthermore, it was demonstrated that in the presence of the attractive interaction $V$ at the nearest sites, the realization of an exotic combination of the $s$-wave and the $p$-wave pairings becomes possible [191].

At present, along with the frequently investigated problem of implementation of the superconducting state in a graphene monolayer within the electronphonon pairing mechanism [192 196], the possibility of the development of Cooper instability in graphene as a result of the electron-electron interactions is being studied actively. In [197], in the $t-J$ model within the renormalized mean-field theory, the possibility of the realization of the superconducting pairing in a graphene monolayer was studied. Both superconductivity with $s$-wave symmetry of the order parameter and a chiral superconductivity with the $d$-wave symmetry (which is described by a two-dimensional representation and breaks the symmetry with respect to time reversal), were shown to be possible. In this case, a significant predominance of $d$-wave pairing over $s$ wave pairing was demonstrated in [197.

When we discuss chirality with respect to the superconducting state, we understand that this state is characterized both by spontaneous time-reversal symmetry breaking and by parity violation (see [198] and 
also review [199]). In other words, this type of superconductivity necessarily includes a linear combination of the two order parameters that belong to a unified higher-dimensional representation of the point symmetry group of the crystal. The chiral superconducting state with the $d$-wave symmetry of the order parameter in graphene is the spin-singlet $d_{x^{2}-y^{2}} \pm i d_{x y^{-}}$ wave state. Since the hexagonal lattice belongs to the symmetry group $C_{6 v}$, the two $d$-wave states make similar contributions but have a phase shift $\pi / 2$ relative to each other. This causes the appearance of a superconducting state in graphene at any finite doping level [198, 199].

The appearance of chiral superconductivity with the $d_{x^{2}-y^{2}} \pm i d_{x y}$-wave symmetry of the order parameter was investigated earlier in cuprates in the presence of magnetic fields [200, 201 and magnetic impurities 202, and also in $2 \mathrm{D}$ superfluid ${ }^{3} \mathrm{He}$ [203. We note that the realization of $d+i d$-wave chiral superconductivity was observed experimentally in the pnictide SrPtAs near the Van Hove filling $n_{V H}$ [204. Note that this compound with $T_{c}=2.4 \mathrm{~K}$ [205] consists of a set of weakly bound layers of PtAs that form a hexagonal lattice.

In 206, in the framework of the $t-J$ model [207] with the Coulomb interaction $V$ between the fermions at neighboring carbon atoms of the hexagonal lattice of graphene (investigated by the method of the functional renormalization group), a triplet $f$-wave pairing and a singlet $d+i d$-wave pairing far from the half-filling were detected. The possibility to realize $d+i d$-wave chiral superconductivity due to the spinfluctuation mechanism was also confirmed by quantum Monte Carlo calculations [208,209].

The situation where the Fermi level is near one of the Van Hove singularities in the density of states of graphene monolayer was considered in [210. It is known that these singularities can enhance magnetic and superconducting fluctuations 211, According to the scenario described in 210, the appearance of Cooper instability is caused by the strong anisotropy of the Fermi contour at the Van Hove filling $n_{V H}$, which is in fact related to the KohnLuttinger mechanism. It is emphasized in 210. that the realization of this mechanism is possible in graphene, since the electron-electron scattering becomes strongly anisotropic and therefore a channel can arise in which the scattering amplitude has an attractive component with a nontrivial angular dependence on the Fermi surface. According to [210, such a Cooper instability in the idealized graphene monolayer is developed predominantly in the $d+i d$-wave channel, and it can lead to critical temperatures up to $T_{c} \sim 10 \mathrm{~K}$, depending on the possibility to tune the level of the chemical potential maximally close to the Van Hove singularity. In [212, the possible coexistence and competition between the Pomeranchuk and Kohn-Luttinger instabilities in graphene monolayer were discussed.

The authors of 213 obtained experimentally a heavily doped monolayer of graphene by a chemical method (different combinations of $\mathrm{K}$ and $\mathrm{Ca}$ were used in [213] as dopants), and investigated the prepared sample by ARPES. It was found that the many-body interactions significantly deform the Fermi surface, leading to the so-called extended Van Hove singularity at the $M$ point of the hexagonal Brillouin zone and inducing a topological transition in the electron system.

Note that the extended Van Hove singularity [214] leads to the divergence in the density of electron states which appears when the energy band of a system is almost flat (up to $1 \mathrm{meV}$ ) in one of the directions of the Brillouin zone. In this case, a set of simple saddle points appears forming a critical line or the socalled extended saddle point 214]. Such an extended saddle point induces a stronger square root-type of the Van Hove divergence in the density of electron states, in contrast to the ordinary saddle point in the energy band, which leads to the usual logarithmic divergence (in the $2 \mathrm{D}$ case) [215]. This square root-type divergence, in turn, can favor a significant increase in the superconducting critical temperature of the transition 214.

Besides the experimental investigation of a heavily doped graphene monolayer, the authors of [213] studied the ground state theoretically and analyzed the competition between the ferromagnetic and superconducting instabilities. The analysis showed that the tendency to superconductivity prevails in this competition, as a result of the strong modulation of the effective interaction along the Fermi contour, i.e., due to electron-electron interactions only. The superconducting instability is then predominantly developed in the $f$-wave channel [213].

The authors of [216, 217] used the method of the functional renormalization group [218 220 to analyze the competition between the superconductivity caused by electron-electron interaction and the phases of spin and charge-density waves at the Van Hove filling in graphene monolayer [221 223$]$. The analysis showed that three Van Hove saddle points with an ideal nesting lead to the domination of the superconducting pairings. The renormalization group analysis has indicated that under these conditions, a spin-singlet superconducting state with the $d+i d-$ wave symmetry type of the order parameter is realized, which spontaneously breaks the symmetry with 
respect to the time reversal and leads to the chiral Andreev states at the boundaries of the sample. In [224, it was stressed that upon a small shift of the Fermi level from the Van Hove singularity, a transition to a spin-density-wave (SDW) phase occurs, and hence the region of the coexistence of superconductivity and the antiferromagnetic ordering in the doped graphene is absent.

It was noted in 225] that the long-range Coulomb interactions can substantially influence the competition between the superconducting phases with different symmetry types of the order parameter in doped graphene. In particular, it was shown in the extended Hubbard model for graphene that far away from the Van Hove singularity, where the $d+$ $i d$-wave spin-singlet pairing is realized, the SDW phase experiences strong competition with the chargedensity-wave (CDW) phase enhanced by the longrange Coulomb interactions, which can favor the realization of the triplet $f$-wave pairing [67] (see below).

The importance of the correct account of the longrange part of the Coulomb interaction when we derive an effective many-particle model for graphene and graphite from $a b$ initio calculations was emphasized in [226]. in fact, from these calculations we can properly determine the values of the partially screened frequency-dependent Coulomb interaction. The Hubbard repulsion in graphene was found to be $U=9.3 \mathrm{eV}$ in agreement with the estimation given in [227, but contradicting the intuitive expectations of a small $U$ and weak coupling $U<W$; it is known 228 that $t_{1}=2.8 \mathrm{eV}$ in graphene. The authors of [226] also calculated the Coulomb repulsion parameters for electrons located on the nearest and next-to-nearest carbon atoms in a graphene monolayer and get $V_{1}=$ $5.5 \mathrm{eV}$ and $V_{2}=4.1 \mathrm{eV}$, respectively. We note that other researchers (see, e.g., [229]) assume that these parameters are much smaller.

The competition of superconducting phases with different symmetry types in a wide range of the concentrations of the charge carriers $1<n \leq n_{V H}$ in the idealized monolayer of doped graphene was investigated in [33, 230]. It was shown that at the intermediate electron densities, the distant Coulomb interactions stimulate superconductivity with the $f$-wave symmetry of the order parameter and upon approaching the Van Hove singularity, the superconducting pairing with the $d+i d$-wave symmetry type is realized [33, 230].

In the hexagonal lattice of graphene, each unit cell contains two carbon atoms; the lattice can therefore is divided into two sublattices, $A$ and $B$ (Fig. 11). The Hamiltonian of the Shubin-Vonsovsky model for a monolayer of graphene, with the electron hoppings

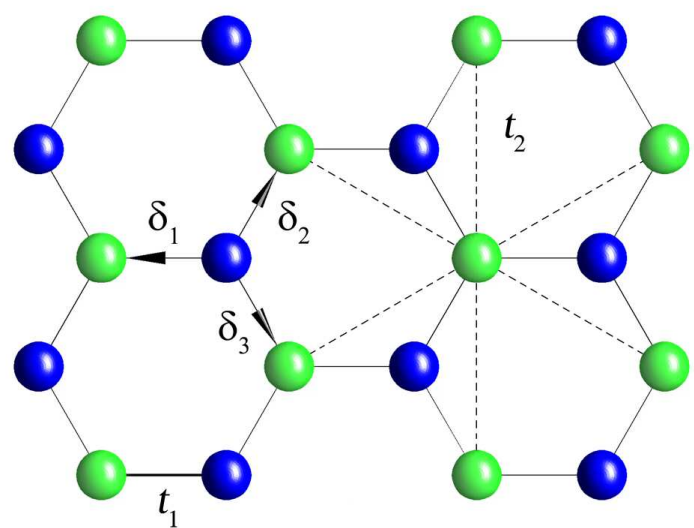

Fig. 11. Crystal structure of a graphene monolayer. The carbon atoms belonging to different sublattices are given by different colors; $\delta_{1}, \delta_{2}$ and $\delta_{3}$ are the vectors in the directions of the nearest neighbors; $t_{1}$ and $t_{2}$ are the hopping integrals between the nearest and next-nearest neighbors.

between the nearest and the next-to-nearest atoms and the Coulomb repulsion of electrons located on the same or different atoms taken into account, in the Wannier representation is given by

$$
\begin{aligned}
& \hat{H}^{\prime}=\hat{H}_{0}^{\prime}+\hat{H}_{\mathrm{int}}, \\
& \hat{H}_{0}^{\prime}=-\mu\left(\sum_{f \sigma} \hat{n}_{f \sigma}^{A}+\sum_{g \sigma} \hat{n}_{g \sigma}^{B}\right) \\
& -t_{1} \sum_{f \delta \sigma}\left(a_{f \sigma}^{\dagger} b_{f+\delta, \sigma}+\text { h.c. }\right) \\
& -t_{2}\left(\sum_{\langle\langle f m\rangle\rangle} a_{f \sigma}^{\dagger} a_{m \sigma}+\sum_{\langle\langle g n\rangle\rangle} b_{g \sigma}^{\dagger} b_{n \sigma}+\text { h.c. }\right), \\
& \hat{H}_{\mathrm{int}}=U\left(\sum_{f} \hat{n}_{f \uparrow}^{A} \hat{n}_{f \downarrow}^{A}+\sum_{g} \hat{n}_{g \uparrow}^{B} \hat{n}_{g \downarrow}^{B}\right) \\
& +V_{1} \sum_{f \delta \sigma \sigma^{\prime}} \hat{n}_{f \sigma}^{A} \hat{n}_{f+\delta, \sigma^{\prime}}^{B} \\
& +\frac{V_{2}}{2}\left(\sum_{\langle\langle f m\rangle\rangle \sigma \sigma^{\prime}} \hat{n}_{f \sigma}^{A} \hat{n}_{m \sigma^{\prime}}^{A}+\sum_{\langle\langle g n\rangle\rangle \sigma \sigma^{\prime}} \hat{n}_{g \sigma}^{B} \hat{n}_{n \sigma^{\prime}}^{B}\right) .
\end{aligned}
$$

Here, $a_{f \sigma}^{\dagger}\left(a_{f \sigma}\right)$ are the creation (annihilation) operators of an electron with the spin projection $\sigma= \pm 1 / 2$ on the site $f$ of the sublattice $A ; \hat{n}_{f \sigma}^{A}=a_{f \sigma}^{\dagger} a_{f \sigma}$ is the operator of the number of fermions on the site $f$ of the sublattice $A$ (and similarly for the sublattice $B)$; the vector $\delta$ connects the nearest-neighbor atoms of the hexagonal lattice; the double angular brackets mean that the summation is carried out only over the next-to-nearest neighbors; $t_{1}$ is the hopping integral 
between the neighboring atoms (hoppings between the different sublattices); $t_{2}$ is the hopping integral between next-to-nearest neighbors (hoppings over one sublattice); $U$ is the parameter of the Hubbard repulsion; and $V_{1}$ and $V_{2}$ are the respective Coulomb repulsions of electrons located on the nearest and next-tonearest carbon atoms. It is assumed that the position of the chemical potential $\mu$ and the number of current carriers $n$ in the graphene monolayer can be controlled by the electric field of the gate electrode.

After passing to the momentum space and carrying out the $u-v$ Bogoliubov transformation,

$$
\begin{aligned}
& a_{\mathbf{k} \sigma}=w_{11 \mathbf{k}} \alpha_{1 \mathbf{k} \sigma}+w_{12 \mathbf{k}} \alpha_{2 \mathbf{k} \sigma} \\
& b_{\mathbf{k} \sigma}=w_{21 \mathbf{k}} \alpha_{1 \mathbf{k} \sigma}+w_{22 \mathbf{k}} \alpha_{2 \mathbf{k} \sigma}
\end{aligned}
$$

where $\alpha_{1 \mathbf{k} \sigma}$ and $\alpha_{2 \mathbf{k} \sigma}$ are the operators that describe the respective dynamics of electrons in the upper and lower bands of the graphene, the Hamiltonian $\hat{H}^{\prime}{ }_{0}$ is diagonalized and, as a result, a well-known expression 25] for the two-band energy spectrum is obtained (see Fig. 10):

$$
E_{1 \mathbf{k}}=t_{1}\left|u_{\mathbf{k}}\right|-t_{2} f_{\mathbf{k}}, \quad E_{2 \mathbf{k}}=-t_{1}\left|u_{\mathbf{k}}\right|-t_{2} f_{\mathbf{k}}
$$

where

$$
\begin{aligned}
& f_{\mathbf{k}}=2 \cos \left(\sqrt{3} k_{y} a\right)+4 \cos \left(\frac{\sqrt{3}}{2} k_{y} a\right) \cos \left(\frac{3}{2} k_{x} a\right) \\
& u_{\mathbf{k}}=\sum_{\delta} e^{i \mathbf{k} \delta}=e^{-i k_{x} a}+2 e^{\frac{i}{2} k_{x} a} \cos \left(\frac{\sqrt{3}}{2} k_{y} a\right) \\
& \left|u_{\mathbf{k}}\right|=\sqrt{3+f_{\mathbf{k}}} .
\end{aligned}
$$

The coefficients of the Bogoliubov transformation are

$$
\begin{aligned}
& w_{1,1}(\mathbf{k})=w_{22}^{*}(\mathbf{k})=\frac{1}{\sqrt{2}} \frac{u_{\mathbf{k}}^{*}}{\left|u_{\mathbf{k}}\right|}, \\
& w_{12}(\mathbf{k})=-w_{21}(\mathbf{k})=-\frac{1}{\sqrt{2}} .
\end{aligned}
$$

In Bogoliubov representation (53), the operator of the interaction in (52) is determined by an expression that includes the operators $\alpha_{1 \mathbf{k} \sigma}$ and $\alpha_{2 \mathbf{k} \sigma}$,

$$
\begin{aligned}
\hat{H}_{\mathrm{int}} & =\frac{1}{N} \sum_{\substack{i j l m \\
\mathbf{k p q} \sigma}} \Gamma_{i j ; l m}^{\|}(\mathbf{k}, \mathbf{p} \mid \mathbf{q}, \mathbf{s}) \alpha_{i \mathbf{k} \sigma}^{\dagger} \alpha_{j \mathbf{p} \sigma}^{\dagger} \alpha_{l \mathbf{q} \sigma} \alpha_{m \mathbf{s} \sigma} \\
& \times \delta(\mathbf{k}+\mathbf{p}-\mathbf{q}-\mathbf{s}) \\
& +\frac{1}{N} \sum_{\substack{i j l m \\
\mathbf{k p q s}}} \Gamma_{i j ; l m}^{\perp}(\mathbf{k}, \mathbf{p} \mid \mathbf{q}, \mathbf{s}) \alpha_{i \mathbf{k} \uparrow}^{\dagger} \alpha_{j \mathbf{p} \downarrow}^{\dagger} \alpha_{l \mathbf{q} \downarrow} \alpha_{m \mathbf{s} \uparrow} \\
& \times \delta(\mathbf{k}+\mathbf{p}-\mathbf{q}-\mathbf{s})
\end{aligned}
$$

where $\Gamma_{i j ; l m}^{\| l}(\mathbf{k}, \mathbf{p} \mid \mathbf{q}, \mathbf{s})$ and $\Gamma_{i j ; l m}^{\perp}(\mathbf{k}, \mathbf{p} \mid \mathbf{q}, \mathbf{s})$ are bare amplitudes, whose form is given below, and $\delta$ is the Dirac delta-function.
The scattering amplitude in the Cooper channel can be calculated using the weak-coupling Born approximation with the hierarchy of the model parameters

$$
W>U>V_{1}>V_{2}
$$

where $W$ is the bandwidth (at $t_{2}=0$ in Eqn (54)) for the upper and lower branches of the energy spectrum of graphene. We restrict again the consideration to the second-order diagrams in the effective interaction of two electrons with opposite values of momentum and spin, using the quantity $U_{\text {eff }}(\mathbf{p}, \mathbf{k})$. Graphically, this quantity is the sum of the diagrams represented in Fig. 2. Assuming that the chemical potential of doped graphene falls into the upper energy band $E_{1 \mathbf{k}}$ and analyzing the conditions of the appearance of the KohnLuttinger superconductivity, we can examine the situation where both the initial and the final momenta belong to the upper band.

Analytically, the effective interaction $U_{\text {eff }}(\mathbf{p}, \mathbf{k})$ is given by

$$
\begin{aligned}
& U_{\mathrm{eff}}(\mathbf{p}, \mathbf{k})=\widetilde{\Gamma}_{0}(\mathbf{p}, \mathbf{k})+\delta U_{\mathrm{eff}}(\mathbf{p}, \mathbf{k}) \\
& \widetilde{\Gamma}_{0}(\mathbf{p}, \mathbf{k})=\Gamma_{i i ; j j}^{\perp}(\mathbf{p},-\mathbf{p} \mid-\mathbf{k}, \mathbf{k}) \\
& \delta U_{\mathrm{eff}}(\mathbf{p}, \mathbf{k})=\frac{1}{N} \sum_{l, m, \mathbf{p}_{1}} \Gamma_{i l ; j m}^{\perp}\left(\mathbf{p}, \mathbf{q}_{2} \mid-\mathbf{k}, \mathbf{p}_{1}\right) \\
& \times \Gamma_{m i ; l j}^{\perp}\left(\mathbf{p}_{1},-\mathbf{p} \mid \mathbf{q}_{2}, \mathbf{k}\right) \chi_{l, m}\left(\mathbf{q}_{2}, \mathbf{p}_{1}\right) \\
& +\frac{2}{N} \sum_{l, m, \mathbf{p}_{1}}\left\{\Gamma_{i m ; l j}^{\perp}\left(\mathbf{p}, \mathbf{p}_{1} \mid \mathbf{q}_{1}, \mathbf{k}\right)\right. \\
& \times\left[\Gamma_{l i ; m j}^{\|}\left(\mathbf{q}_{1},-\mathbf{p} \mid \mathbf{p}_{1},-\mathbf{k}\right)-\Gamma_{l i ; j m}^{\|}\left(\mathbf{q}_{1},-\mathbf{p} \mid-\mathbf{k}, \mathbf{p}_{1}\right)\right] \\
& +\Gamma_{l i ; j m}^{\perp}\left(\mathbf{q}_{1},-\mathbf{p} \mid-\mathbf{k}, \mathbf{p}_{1}\right) \\
& \left.\times\left[\Gamma_{i m ; j l}^{\|}\left(\mathbf{p}, \mathbf{p} \mathbf{p}_{1} \mid \mathbf{k}, \mathbf{q}_{1}\right)-\Gamma_{i m ; l j}^{\|}\left(\mathbf{p}, \mathbf{p}_{1} \mid \mathbf{q}_{1}, \mathbf{k}\right)\right]\right\} \chi_{l, m}\left(\mathbf{q}_{1}, \mathbf{p}_{1}\right)
\end{aligned}
$$

where the expressions

$$
\begin{aligned}
\Gamma_{i j ; l m}^{l \mid}(\mathbf{k}, \mathbf{p} \mid \mathbf{q}, \mathbf{s}) & =\frac{1}{2}\left(V_{i j ; l m}(\mathbf{k}, \mathbf{p} \mid \mathbf{q}, \mathbf{s})\right. \\
& \left.+V_{j i ; m l}(\mathbf{p}, \mathbf{k} \mid \mathbf{s}, \mathbf{q})\right) \\
V_{i j ; l m}(\mathbf{k}, \mathbf{p} \mid \mathbf{q}, \mathbf{s}) & =V_{1} u_{\mathbf{q}-\mathbf{p}} w_{i 1}(\mathbf{k}) w_{j 2}(\mathbf{p}) w_{l 2}^{*}(\mathbf{q}) w_{m 1}^{*}(\mathbf{s}) \\
& +\frac{V_{2}}{2} f_{\mathbf{q}-\mathbf{p}}\left(w_{i 1}(\mathbf{k}) w_{j 1}(\mathbf{p}) w_{l 1}^{*}(\mathbf{q}) w_{m 1}^{*}(\mathbf{s})\right. \\
& \left.+w_{i 2}(\mathbf{k}) w_{j 2}(\mathbf{p}) w_{l 2}^{*}(\mathbf{q}) w_{m 2}^{*}(\mathbf{s})\right)
\end{aligned}
$$

describe the strength of the interaction of fermions with parallel projections of spin, and the expressions

$$
\begin{aligned}
& \Gamma_{i j ; l m}^{\perp}(\mathbf{k}, \mathbf{p} \mid \mathbf{q}, \mathbf{s})=U_{i j ; l m}(\mathbf{k}, \mathbf{p} \mid \mathbf{q}, \mathbf{s}) \\
& \quad+V_{i j ; l m}(\mathbf{k}, \mathbf{p} \mid \mathbf{q}, \mathbf{s})+V_{j i ; m l}(\mathbf{p}, \mathbf{k} \mid \mathbf{s}, \mathbf{q}), \\
& U_{i j ; l m}(\mathbf{k}, \mathbf{p} \mid \mathbf{q}, \mathbf{s})=U\left(w_{i 1}(\mathbf{k}) w_{j 1}(\mathbf{p}) w_{l 1}^{*}(\mathbf{q}) w_{m 1}^{*}(\mathbf{s})\right. \\
& \left.\quad+w_{i 2}(\mathbf{k}) w_{j 2}(\mathbf{p}) w_{l 2}^{*}(\mathbf{q}) w_{m 2}^{*}(\mathbf{s})\right)
\end{aligned}
$$


correspond to the interaction of Fermi quasiparticles with antiparallel projections of spin. In expressions (61)-65), the indices $i=j=1$, and the indices $l$ and $m$ can take the values 1 or 2 for upper and lower bands. We introduce the generalized susceptibilities

$$
\chi_{l, m}(\mathbf{k}, \mathbf{p})=\frac{f\left(E_{l \mathbf{k}}\right)-f\left(E_{m \mathbf{p}}\right)}{E_{m \mathbf{p}}-E_{l \mathbf{k}}}
$$

where $n_{F}(x)=\{\exp [(x-\mu) / T]+1\}^{-1}$ is the FermiDirac distribution function, and the energies $E_{i \mathbf{k}}$ are defined in (54). For compactness, the following notation is introduced in Eqn (61) for the combinations of the momenta:

$$
\mathbf{q}_{1}=\mathbf{p}_{1}+\mathbf{p}-\mathbf{k}, \quad \mathbf{q}_{2}=\mathbf{p}_{1}-\mathbf{p}-\mathbf{k}
$$

Just as in the case of the Shubin-Vonsovsky model, on the square lattice (see Section $\mathbf{6}$.), the problem of the Cooper instability in graphene monolayer can be reduced to the eigenvalue problem

$$
\frac{3 \sqrt{3}}{8 \pi^{2}} \oint_{\varepsilon_{\mathbf{q}}=\mu} \frac{d \hat{\mathbf{q}}}{v_{F}(\hat{\mathbf{q}})} U_{\mathrm{eff}}(\hat{\mathbf{p}}, \hat{\mathbf{q}}) \Delta(\hat{\mathbf{q}})=\lambda \Delta(\hat{\mathbf{p}}),
$$

where the integration is carried out over the contour shown in Fig. 10].

To solve Eqn (68), we represent its kernel as a superposition of the eigenfunctions, each belonging to one of the irreducible representations of the symmetry group $C_{6 v}$ of the hexagonal lattice. As it is known, this symmetry group has six irreducible representations [121]: four one-dimensional and two twodimensional. For each representation, Eqn (68) has a solution with its own effective coupling constant $\lambda$. We use the following notation for the classification of the symmetries of the order parameter, namely, representation $A_{1}$ corresponds to $s$-wave symmetry; $A_{2}$ to extended $s$-wave pairing; $B_{1}$ and $B_{2}$ to $f$-wave symmetry; $E_{1}$ to $p+i p$-wave symmetry, and $E_{2}$ to $d+i d$-wave symmetry.

For each irreducible representation $\nu=$ $A_{1}, A_{2}, B_{1}, B_{2}, E_{1}, E_{2}$, we search the solution to Eqn (43) in the form

$$
\Delta^{(\nu)}(\phi)=\sum_{m} \Delta_{m}^{(\nu)} g_{m}^{(\nu)}(\phi)
$$

where $m$ is the number of an eigenfunction of the representation $\nu$, and $\phi$ is the angle that determines the direction of the momentum $\mathbf{p}$ relative to the axis $p_{x}$. Explicitly, the orthonormalized functions $g_{m}^{(\nu)}(\phi)$ are given by

$$
\begin{aligned}
& A_{1} \rightarrow g_{m}^{(s)}(\phi)=\frac{1}{\sqrt{\left(1+\delta_{m 0}\right)}} \cos 6 m \phi, \quad m \in[0, \infty), \\
& A_{2} \rightarrow g_{m}^{\left(A_{2}\right)}(\phi)=\frac{1}{\sqrt{\pi}} \sin (6 m+6) \phi, \\
& B_{1} \rightarrow g_{m}^{\left(f_{1}\right)}(\phi)=\frac{1}{\sqrt{\pi}} \sin (6 m+3) \phi, \\
& B_{2} \rightarrow g_{m}^{\left(f_{2}\right)}(\phi)=\frac{1}{\sqrt{\pi}} \cos (6 m+3) \phi, \\
& E_{1} \rightarrow g_{m}^{(p+i p)}(\phi)=\frac{1}{\sqrt{\pi}}(A \sin (2 m+1) \phi+ \\
& \quad+B \cos (2 m+1) \phi), \\
& E_{2} \rightarrow \quad g_{m}^{(d+i d)}(\phi)=\frac{1}{\sqrt{\pi}}(A \sin (2 m+2) \phi+ \\
& \quad+B \cos (2 m+2) \phi) .
\end{aligned}
$$

Here, for the two-dimensional representations $E_{1}$ and $E_{2}$, the indices $m$ range over to values such that the coefficients $2 m+1$ and $2 m+2$ are not multiples of 3 .

Figure 12a shows the phase diagram of the KohnLuttinger superconducting state when the energy spectrum of the monolayer is described by only one hopping parameter $\left(t_{1} \neq 0, t_{2}=0\right)$, the Hubbard repulsion, $U=2$ (hereinafter, all parameters are given in the units of $\left.\left|t_{1}\right|\right)$, as well as the Coulomb repulsion of electrons located only on the neighboring carbon atoms $\left(V_{1} \neq 0, V_{2}=0\right)$ are taken into account.

We see from fig. 12 a that the phase diagram contains three regions. At low electron densities $n$, superconductivity with the $d+i d$-wave symmetry of the order parameter is realized. At intermediate electron concentrations, a superconducting $f$-wave pairing takes place. The contribution to it is determined by the harmonics $g_{m}^{\left(f_{1}\right)}(\phi)=\frac{1}{\sqrt{\pi}} \sin (6 m+3) \phi$, while a contribution from the harmonics $g_{m}^{\left(f_{2}\right)}(\phi)=$ $\frac{1}{\sqrt{\pi}} \cos (6 m+3) \phi$ is absent. At larger densities $n$, a region of the chiral $d+i d$-wave pairing appears again [197, 210, 216, 225. With an increase in the parameter of the intersite Coulomb interaction $V_{1}$, in the region of small $n$, the $d+i d$-wave pairing is suppressed, and the pairing with $f$-wave symmetry of the order parameter is realized. The thin curves in Fig. 12 correspond to the lines of constant $|\lambda|$. It can be seen that at the approaching the Van Hove filling $n_{V H}$ (solid curve in Fig. 13), the values of the effective coupling constant reach quite reasonable values $|\lambda|=0.1$.

Here, again to avoid the summation of parquet diagrams 62, 65, 66, only the regions of electron concentrations that are not to close to the Van Hove singularity in the density of electronic states of graphene are analyzed (see Fig. 13). For this reason, the bound- 
a)

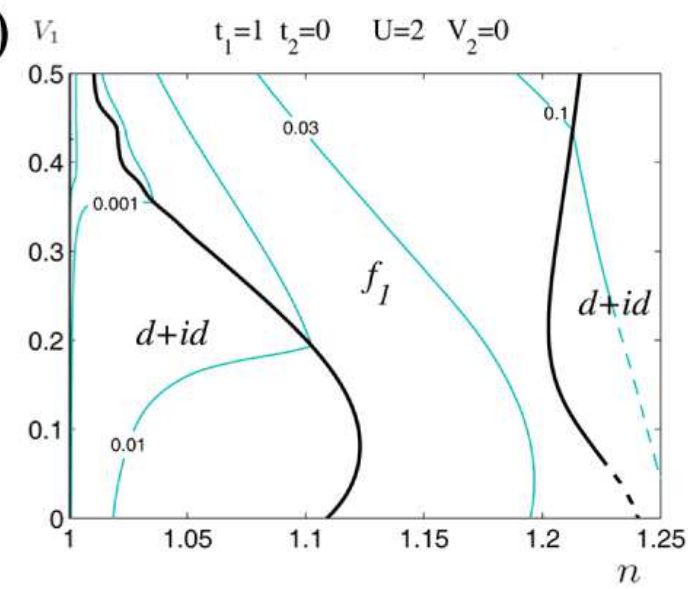

b) $v_{1}$

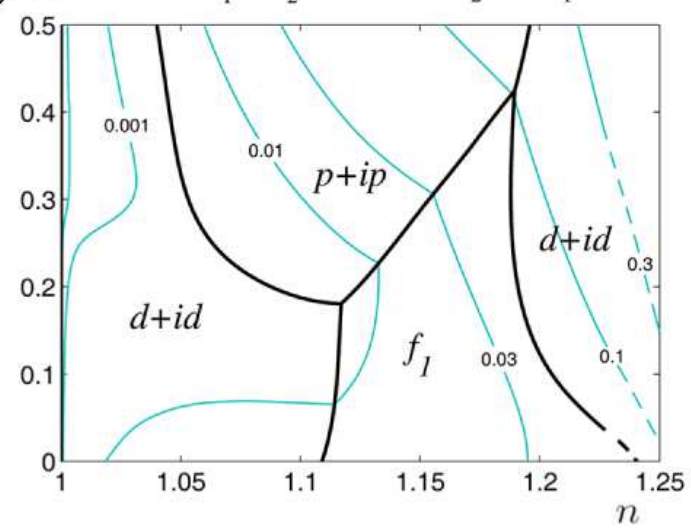

Fig. 12. The " $n-V_{1}$ " phase diagram of the superconducting state of the graphene monolayer at $t_{2}=0, U=2$ for $V_{2}=0$ (a) and $V_{2}=0.6 V_{1}$ (b) (all the parameters in the units of $\left|t_{1}\right|$ ). The thin lines are lines of constant $|\lambda|[231$.

aries between the different regions of the realization of the Kohn-Luttinger superconducting pairing and the lines of the constant $|\lambda|$ located very close to the Van Hove singularity are depicted in the phase diagram as dashed curves.

When we take into account the long-range Coulomb interactions of electrons $V_{2}$ on the hexagonal lattice of graphene, a qualitative change occurs in the phase diagram of the superconducting state [231]. This can be seen from Fig. 12b, which was obtained at a fixed relation between the parameters of the long-range Coulomb interactions $V_{2}=0.6 V_{1}$. In this case, a suppression occurs of the wide region of the superconducting state with the $f$-wave symmetry at the intermediate electron densities, and a superconducting pairing with the $p+i p$-wave symmetry is realized. Furthermore, an account of $V_{2}$ leads to an increase in the values of the effective coupling constant to $|\lambda|=0.3$.

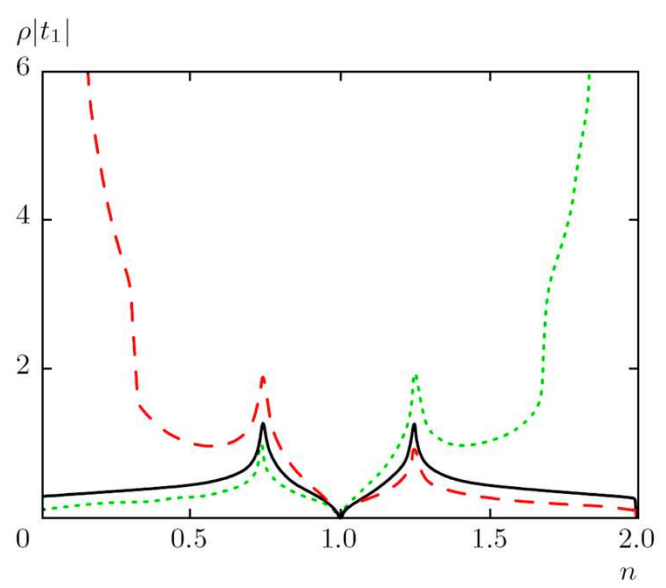

Fig. 13. Modification of the electron density of states for the graphene monolayer upon switching of the hoppings to the next-nearest atoms for $t_{2}=0$ (solid curve), $t_{2}=-0.2\left|t_{1}\right|$ (dashed curve), and $t_{2}=0.2\left|t_{1}\right|$ (dotted curve) 33.

Account for the electron hoppings to the next-tonearest carbon atoms $t_{2}$ in the graphene monolayer does not qualitatively affect the competition between the superconducting phases with different symmetry types, as can be seen from Fig 12b 33. This behavior of the system is explained by the fact that the hoppings with $t_{2}>0$ or $t_{2}<0$ do not cause an essential modification of the density of states of the monolayer in the ranges of concentrations of charge carriers between the Dirac point and both Van Hove singularities (see Fig. 13). But when we take the hoppings $t_{2}$ into account we get an increase in the absolute values of the effective interaction and, consequently, the realization of the higher critical temperatures in the idealized graphene monolayer 33 .

We note that the Kohn-Luttinger superconductivity (and the corresponding value of $|\lambda|$ ) in graphene is never connected with the Dirac points. Calculations show that in the vicinity of these points, where a linear approximation to the energy spectrum of the monolayer (and a parabolic approximation for the spectrum of graphene bilayer, see Section (9) works well, the density of states is very low, and the value of the effective coupling constant $|\lambda|$ is less than $10^{-2}$. Larger values of $|\lambda|$, which indicate the development of a Cooper instability, appear at electron densities $n>1.15$. However, at these concentrations, the energy spectrum of the monolayer along the $K M$ direction of the Brillouin zone (Fig. 14) already differs significantly from the Dirac approximation 231.

In Refs 232, 233, the possibility of Cooper pairing in graphene was investigated in the opposite limit of strong coupling, $U \gg t$ [226], which is based on 
the kinematic mechanism of superconductivity and using the diagram technique for the Hubbard operators [56, 152, 153]. In particular, a phase diagram for the superconducting ordering was constructed and the BCS coupling constant was calculated depending on the filling of the $\pi$ or $\sigma$ shell.

\section{Enhancement of superconductivity in an idealized graphene bilayer}

As far as the electronic properties of graphene depend on the number of carbon layers [234], we therefore analyze the possibility of the development of superconducting instability in an idealized graphene bilayer [231, 235, whose crystalline structure is shown in Fig. 15. The energy band structure of a monolayer is characterized by a linear dispersion near the Dirac points, while the bilayer has a quadratic energy spectrum in the low-energy limit (see 236 238, and also reviews [239,240]).

The authors of 241] examined the effect of the interplanar electron hopping in bilayer graphene and graphite on the formation of the superconducting phase. Assuming that the Hubbard interaction is attractive and leads to a superconducting $s$-wave pairing, the authors of [241] showed in the mean-field approximation that the interplanar hopping increases the critical temperature $T_{c}$ of the superconducting transition at low values of the chemical potential.

The authors of 242 investigated the possibility of the realization of the superconducting phase in the mean-field approximation in the framework of the $t-J$

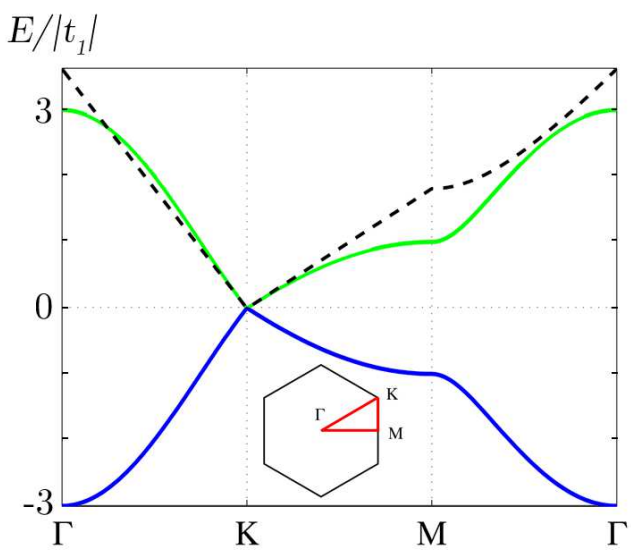

Fig. 14. Comparison of the energy spectrum of a graphene monolayer determined by Eqn (54) (blue and green solid lines) and of the spectrum obtained in the Dirac approximation (black dashed line). The insert depicts the contour for moving around the Brillouin zone.

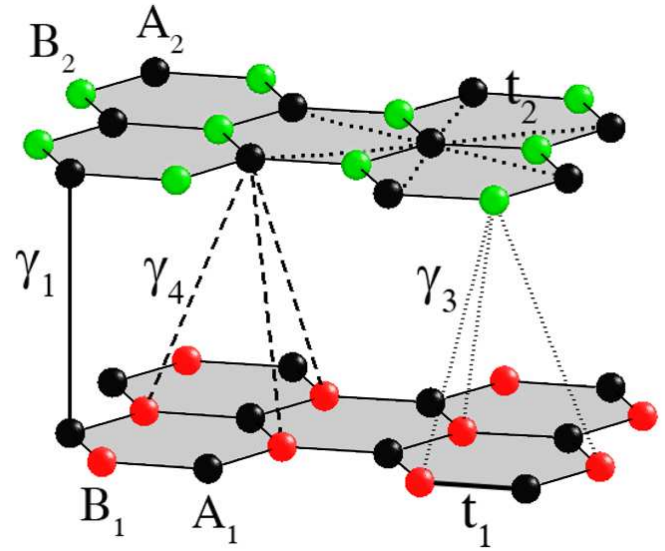

Fig. 15. Crystal structure of the graphene bilayer. Carbon atoms $A_{1}$ and $B_{1}$ in the lower layer are shown by red and black circles; atoms $A_{2}$ and $B_{2}$ in the upper layer are shown by black and green circles. Intralayer electron hoppings are marked by $t_{1}$ and $t_{2}$; $\gamma_{1}, \gamma_{3}$, and $\gamma_{4}$ show the interplane hoppings 231.

model for a graphene bilayer with the direct interlayer hopping $\gamma_{1}$, (see Fig. 15) and the superexchange interaction caused by the strong Hubbard repulsion $U$ of electrons. It was shown that in the bilayer with moderate doping and for the low-energy scales, the $d+i d$-wave pairing is predominant. In [243], using the same model the authors discussed the coexistence of chiral superconductivity with the $d+i d$-wave symmetry and antiferromagnetism near the half-filling for the graphene bilayer.

The authors of [244,245] studied the exotic superconductivity mechanism based on the interlayer pairing of chiral electrons in a graphene bilayer, which leads to anomalous thermodynamic properties. According to the conclusions in [244, 245], this mechanism of superconductivity in a graphene bilayer is quite similar to color superconductivity 246] (based on the pairing of quarks in high-density quark matter at low temperatures) and to gapless states in nuclear matter 247.

The authors of [248 used the random phase approximation (RPA) to calculate the screening of Coulomb interaction in a graphene bilayer in both doped and undoped regimes. They found that the static polarization operator of a doped bilayer contains the Kohn anomaly that is considerably larger than in the case of graphene monolayer or a 2D electron gas. As it was already noted in Section [2, the singular part of the polarization operator, or the Kohn anomaly, favors an effective attraction of two particles ensuring a contribution that (for the orbital angular momenta of the pair $l \neq 0$ ) always exceeds the re- 
pulsive contribution caused by the regular part of the polarization operator. Thus, the authors of [248] concluded that in the idealized bilayer, it is possible to expect an increase in the critical temperatures $T_{c}$ of the superconducting transition in comparison with $T_{c}$ in the idealized graphene monolayer.

According to the results in [249], the ferromagnetic instability near the Van Hove singularities prevails over the Kohn-Luttinger superconductivity. Nevertheless, in [250], the possibility of the superconducting pairing in the repulsive case on the hexagonal lattice for the graphene bilayer was investigated within the renormalization group formalism in the weak-coupling regime far away from the half-filling. Although the utilization of the renormalization group approach in this regime can be substantiated only formally [250], the authors discovered, a chiral $d$-wave type superconductivity besides the $f$-wave type of the superconducting pairings. Estimating the critical temperature of the superconducting transition in the idealized system, the authors of [250] obtained $T_{c} \sim 1 \mathrm{~K}$ and noted that the critical temperature can be lower if we take into account the electron scattering on impurities.

In [231, 235], the authors investigated in details the influence of the Coulomb interaction of the Dirac fermions on the formation of the Kohn-Luttinger superconducting state in doped bilayer graphene neglecting the van der Waals potential of the substrate and the role of impurities. Within the ShubinVonsovsky model in the weak-coupling Born approximation, taking into account the Hubbard, interatomic (inside the layer), and interlayer Coulomb interactions of electrons, a phase diagram of superconducting state was constructed. It has been shown that the Kohn-Luttinger polarization contributions on the second order of the perturbation theory and the longrange intraplane Coulomb interactions substantially influence the competition between the superconducting phases with the $f$-, $p+i p$-, and $d+i d$-wave symmetries. It has been demonstrated that the interlayer Coulomb interaction can lead to an increase in the critical temperature of the superconducting transition. Now, we discuss the results of the calculations performed in 231,235] in more details.

We consider an idealized graphene bilayer, assuming that two monolayers are located according to the $A B$-stacking, i.e., one layer is turned through $60^{\circ}$ relative to the other one 236,240]. In this case, we choose an arrangement of the sublattices in the layers in such a way that the sites from the different layers located on top of one another will be refered to the sublattices $A_{1}$ and $A_{2}$, and the remaining sites to the sublattices $B_{1}$ and $B_{2}$ (see Fig. 15). In this case, the Hamiltonian of the Shubin-Vonsovsky model for bilayer graphene taking into account the electron hoppings between the nearest and next-to-nearest atoms, the Coulomb repulsion of electrons located on the same and on different atoms of one layer, as well as with the interlayer Coulomb interaction, has the following form in the Wannier representation:

$$
\begin{aligned}
\hat{H}^{\prime} & =\hat{H}^{\prime}{ }_{0}+\hat{H}_{\mathrm{int}}, \\
\hat{H}_{0}^{\prime} & =(\varepsilon-\mu)\left(\sum_{i f \sigma} \hat{n}_{i f \sigma}^{A}+\sum_{i g \sigma} \hat{n}_{i g \sigma}^{B}\right) \\
& -t_{1} \sum_{f \delta \sigma}\left(a_{1 f \sigma}^{\dagger} b_{1, f+\delta, \sigma}+a_{2 f \sigma}^{\dagger} b_{2, f-\delta, \sigma}+\text { h.c. }\right) \\
& -t_{2} \sum_{i \sigma}\left(\sum_{\langle\langle f m\rangle} a_{i f \sigma}^{\dagger} a_{i m \sigma}+\sum_{\langle\langle g n\rangle\rangle} b_{i g \sigma}^{\dagger} b_{i n \sigma}+\text { h.c. }\right) \\
& -\gamma_{1} \sum_{f \sigma}\left(a_{1 f \sigma}^{\dagger} a_{2 f \sigma}+\text { h.c. }\right) \\
& -\gamma_{3} \sum_{g \delta \sigma}\left(b_{1 g \sigma}^{\dagger} b_{2, g+\delta, \sigma}+\text { h.c. }\right) \\
& -\gamma_{4} \sum_{f \delta \sigma}\left(a_{1 f \sigma}^{\dagger} b_{2, f-\delta, \sigma}+a_{2 f \sigma^{\dagger}}^{\dagger} b_{1, f+\delta, \sigma}+\text { h.c. }\right),(72) \\
& +G_{4} \sum_{f \delta \sigma \sigma^{\prime}}\left(\hat{n}_{1 f \sigma}^{A} \hat{n}_{2, f-\delta, \sigma^{\prime}}^{B}+\hat{n}_{2 f \sigma}^{A} \hat{n}_{1, f+\delta, \sigma^{\prime}}^{B}\right) .(73) \\
& +\frac{V_{2}}{2} \sum_{i \sigma \sigma^{\prime}}\left(\sum_{\langle\langle f m\rangle\rangle}^{A} \hat{n}_{i f \sigma}^{A} \hat{n}_{2 f \sigma^{\prime}}^{A}+G_{i m \sigma^{\prime}}+\sum_{j \delta \sigma \sigma^{\prime}} \sum_{i\langle g n\rangle\rangle} \hat{n}_{1 g \sigma}^{B} \hat{n}_{2, g+\delta, \sigma^{\prime}}^{B} \hat{n}_{i n \sigma^{\prime}}^{B}\right) \\
& \left.+V_{1} \sum_{i f} \hat{n}_{i f \uparrow}^{A} \hat{n}_{i f \downarrow}^{A}+\sum_{i g}^{A} \hat{n}_{i g \uparrow}^{B} \hat{n}_{1, f+\delta, \sigma^{\prime}}^{B}+\hat{n}_{2 f \sigma}^{A} \hat{n}_{2, f-\delta, \sigma^{\prime}}^{B}\right)
\end{aligned}
$$

The notations used here are similar to ones used for monolayer Hamiltonian (52). In Hamiltonian (71), the index $i=1,2$ stands for the number of a layer. The vector $\delta(-\delta)$ connects the nearest-neighbor atoms of the hexagonal lattice of the lower (upper) layer. It is assumed that the on-site energies are $\varepsilon_{A i}=\varepsilon_{B i}=\varepsilon$. The symbols $\gamma_{1}, \gamma_{3}$ and $\gamma_{4}$ are the parameters of interlayer electron hoppings (see Fig. 15), and $G_{1}, G_{3}$ and $G_{4}$ are the interlayer Coulomb interactions.

As in the case of a monolayer (see Section \$.), the Hamiltonian $\hat{H}_{0}^{\prime}$ is diagonalized by the Bogoliubov transformation

$$
\begin{aligned}
\alpha_{i \mathbf{k} \sigma} & =w_{i 1}(\mathbf{k}) a_{1 \mathbf{k} \sigma}+w_{i 2}(\mathbf{k}) a_{2 \mathbf{k} \sigma} \\
& +w_{i 3}(\mathbf{k}) b_{1 \mathbf{k} \sigma}+w_{i 4}(\mathbf{k}) b_{2 \mathbf{k} \sigma}, \quad i=1,2,3,4
\end{aligned}
$$

and is reduced to the form 


$$
\hat{H}_{0}^{\prime}=\sum_{i=1}^{4} \sum_{\mathbf{k} \sigma} E_{i \mathbf{k}} \alpha_{i \mathbf{k} \sigma}^{\dagger} \alpha_{i \mathbf{k} \sigma}
$$

Since the results of $a b$ initio calculations [251, 252] performed for graphite indicate a very low value of the parameter of the interlayer hopping $\gamma_{4}$, we assume that $\gamma_{4}=0$. In this case, the four-band energy spectrum of bilayer graphene is given by

$$
\begin{aligned}
E_{i \mathbf{k}}= & \varepsilon \pm \sqrt{A_{\mathbf{k}} \pm \sqrt{B_{\mathbf{k}}}}-t_{2} f_{\mathbf{k}} \\
A_{\mathbf{k}}= & \frac{1}{4}\left(2 a^{2}+4\left|b_{\mathbf{k}}\right|^{2}+2\left|d_{\mathbf{k}}\right|^{2}\right) \\
B_{\mathbf{k}}= & \frac{1}{4}\left(\left|d_{\mathbf{k}}\right|^{2}\left(\left|d_{\mathbf{k}}\right|^{2}-2 a^{2}+4\left|b_{\mathbf{k}}\right|^{2}\right)+a^{4}+4 a^{2}\left|b_{\mathbf{k}}\right|^{2}\right. \\
& \left.+4 a b_{\mathbf{k}}^{2} d_{\mathbf{k}}+4 a b_{\mathbf{k}}^{* 2} d_{\mathbf{k}}^{*}\right) \\
a= & \gamma_{1}, \quad b_{\mathbf{k}}=t_{1} u_{\mathbf{k}}, \quad d_{\mathbf{k}}=\gamma_{3} u_{\mathbf{k}} .
\end{aligned}
$$

The conditions for the appearance of the KohnLuttinger superconductivity in the graphene bilayer in the Born weak-coupling approximation are analyzed using the hierarchy of the model parameters

$$
W>U>V_{1}>V_{2}>G_{1}>G_{3}, G_{4}
$$

(where $W$ is the bandwidth of the graphene bilayer), according to the general scheme presented in Section $\$$. Upon doping of the bilayer, the chemical potential is assumed to be located inside the two upper energy bands, $E_{1 \mathbf{k}}$ and $E_{2 \mathbf{k}}$, as it is shown in Fig. 16a. In this case, if $\gamma_{1} \neq 0$ and $\mu>\gamma_{1}$, in the vicinity of each Dirac point at the electron densities $1<n<n_{V H}$ (where $n$ is the electron concentration calculated per number of atoms of one layer), the Fermi contour consists of two lines, as it is shown in Fig. 16b. The initial and the final momenta of electrons in the Cooper channel then also belong to the two upper bands and, consequently, the indices $i$ and $j$ in the Kohn-Luttinger diagrams in the case of a bilayer (see Fig. 2) take the values 1 or 2. As a result, we arrive at expression (61) for the effective interaction of electrons in the Cooper channel, in which the bare amplitudes for the graphene bilayer are given by
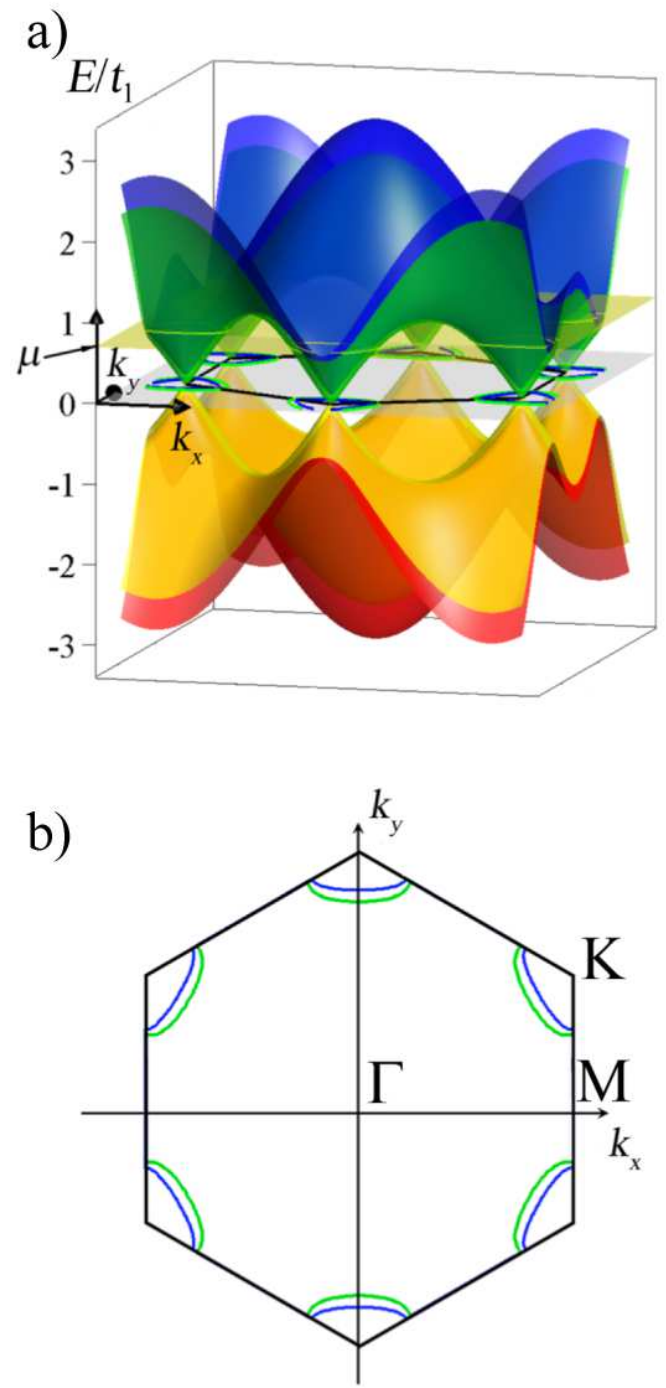

Fig. 16. (a) Energy structure of the graphene bilayer near the Dirac points and (b) the formation of a multisheet Fermi contour at $t_{2}=0, \gamma_{1}=0.12, \gamma_{3}=0.1$ and $\mu=0.7$ (all the parameters are in the units of $\left.\left|t_{1}\right|\right)$ 231.

$$
\begin{aligned}
\Gamma_{i j ; l m}^{||} & (\mathbf{k}, \mathbf{p} \mid \mathbf{q}, \mathbf{s}) \\
& =\frac{1}{2}\left(V_{i j ; l m}(\mathbf{k}, \mathbf{p} \mid \mathbf{q}, \mathbf{s})+V_{j i ; m l}(\mathbf{p}, \mathbf{k} \mid \mathbf{s}, \mathbf{q})\right. \\
& +G_{i j ; l m}^{(1)}(\mathbf{k}, \mathbf{p} \mid \mathbf{q}, \mathbf{s})+G_{j i ; m l}^{(1)}(\mathbf{p}, \mathbf{k} \mid \mathbf{s}, \mathbf{q}) \\
& +G_{i j ; l m}^{(3)}(\mathbf{k}, \mathbf{p} \mid \mathbf{q}, \mathbf{s})+G_{j i ; m l}^{(3)}(\mathbf{p}, \mathbf{k} \mid \mathbf{s}, \mathbf{q}) \\
& \left.+G_{i j ; l m}^{(4)}(\mathbf{k}, \mathbf{p} \mid \mathbf{q}, \mathbf{s})+G_{j i ; m l}^{(4)}(\mathbf{p}, \mathbf{k} \mid \mathbf{s}, \mathbf{q})\right), \\
V_{i j ; l m} & (\mathbf{k}, \mathbf{p} \mid \mathbf{q}, \mathbf{s})=V_{1}\left(u_{\mathbf{q}-\mathbf{p}} w_{i 1}(\mathbf{k}) w_{j 3}(\mathbf{p}) w_{l 3}^{*}(\mathbf{q}) w_{m 1}^{*}(\mathbf{s})\right. \\
& \left.+u_{\mathbf{q}-\mathbf{p}}^{*} w_{i 2}(\mathbf{k}) w_{j 4}(\mathbf{p}) w_{l 4}^{*}(\mathbf{q}) w_{m 2}^{*}(\mathbf{s})\right) \\
& +\frac{V_{2}}{2} \sum_{r=1}^{4} f_{\mathbf{q}-\mathbf{p}} w_{i r}(\mathbf{k}) w_{j r}(\mathbf{p}) w_{l r}^{*}(\mathbf{q}) w_{m r}^{*}(\mathbf{s}),
\end{aligned}
$$




$$
\begin{aligned}
& G_{i j ; l m}^{(1)}(\mathbf{k}, \mathbf{p} \mid \mathbf{q}, \mathbf{s})=G_{1} w_{i 1}(\mathbf{k}) w_{j 2}(\mathbf{p}) w_{l 2}^{*}(\mathbf{q}) w_{m 1}^{*}(\mathbf{s}), \\
& G_{i j ; l m}^{(3)}(\mathbf{k}, \mathbf{p} \mid \mathbf{q}, \mathbf{s}) \\
& =G_{3} u_{\mathbf{q}-\mathbf{p}} w_{i 3}(\mathbf{k}) w_{j 4}(\mathbf{p}) w_{l 4}^{*}(\mathbf{q}) w_{m 3}^{*}(\mathbf{s}), \\
& G_{i j ; l m}^{(4)}(\mathbf{k}, \mathbf{p} \mid \mathbf{q}, \mathbf{s})=G_{4}\left(u_{\mathbf{q}-\mathbf{p}}^{*} w_{i 1}(\mathbf{k}) w_{j 4}(\mathbf{p}) w_{l 4}^{*}(\mathbf{q}) w_{m 1}^{*}(\mathbf{s})\right. \\
& \left.+u_{\mathbf{q}-\mathbf{p}} w_{i 2}(\mathbf{k}) w_{j 3}(\mathbf{p}) w_{l 3}^{*}(\mathbf{q}) w_{m 2}^{*}(\mathbf{s})\right), \\
& \Gamma_{i j ; l m}^{\perp}(\mathbf{k}, \mathbf{p} \mid \mathbf{q}, \mathbf{s})=U_{i j ; l m}(\mathbf{k}, \mathbf{p} \mid \mathbf{q}, \mathbf{s}) \\
& +V_{i j ; l m}(\mathbf{k}, \mathbf{p} \mid \mathbf{q}, \mathbf{s})+V_{j i ; m l}(\mathbf{p}, \mathbf{k} \mid \mathbf{s}, \mathbf{q}) \\
& +G_{i j ; l m}^{(1)}(\mathbf{k}, \mathbf{p} \mid \mathbf{q}, \mathbf{s})+G_{j i ; m l}^{(1)}(\mathbf{p}, \mathbf{k} \mid \mathbf{s}, \mathbf{q}) \\
& +G_{i j ; l m}^{(3)}(\mathbf{k}, \mathbf{p} \mid \mathbf{q}, \mathbf{s})+G_{j i ; m l}^{(3)}(\mathbf{p}, \mathbf{k} \mid \mathbf{s}, \mathbf{q}) \\
& +G_{i j ; l m}^{(4)}(\mathbf{k}, \mathbf{p} \mid \mathbf{q}, \mathbf{s})+G_{j i ; m l}^{(4)}(\mathbf{p}, \mathbf{k} \mid \mathbf{s}, \mathbf{q}), \\
& U_{i j ; l m}(\mathbf{k}, \mathbf{p} \mid \mathbf{q}, \mathbf{s}) \\
& =U \sum_{r=1}^{4} w_{i r}(\mathbf{k}) w_{j r}(\mathbf{p}) w_{l r}^{*}(\mathbf{q}) w_{m r}^{*}(\mathbf{s}) .
\end{aligned}
$$

Here, the indices $l$ and $m$ can take the values $1,2,3$, or 4 .

As it was already noted in the case of the monolayer, there is no common opinion in the literature concerning the values of the intraplane and interplane Coulomb interaction parameters in a graphene bilayer [226,243]. In our calculations, we used the hierarchy of parameters given by (77), which allows the application of the Born weak-coupling approximation. For the parameters of the interlayer hopping $\gamma_{1}$ and $\gamma_{3}$, values close to those determined for graphite in Refs 251,252, are used.

Let us consider the superconducting phase diagram of the graphene bilayer and its modifications by different interactions. First of all, we note that at $\gamma_{1}=\gamma_{3}=\gamma_{4}=0$ and $G_{1}=G_{3}=G_{4}=0$, when the graphene bilayer represents two isolated monolayers, a limiting transition to the results obtained for the monolayer in Section 8 is checked in the numerical calculations. Choosing the interlayer electron hopping parameters as $\gamma_{1}=0.12, \gamma_{3}=0.1$ (hereinafter, all parameters are taken in the units of $\left.\left|t_{1}\right|\right)$, with all the other parameters having the same values as in Fig. 12, we do not get considerable changes in the phase diagram of the graphene bilayer. Including the Coulomb interaction $G_{1}$, we get only a weak shift of the boundaries of the $f_{1}$ and $d+i d$-wave pairing in the phase diagram of Fig. 12, Moreover, this inclusion does not affect the absolute values of $\lambda$.

Figure 17 demonstrates the influence of the interlayer Coulomb interactions $G_{3}$ and $G_{4}$ on the phase diagram of the graphene bilayer. This diagram was obtained using the set of parameters $t_{2}=0, \gamma_{1}=$ $0.12, \gamma_{3}=0.1, U=2$ and $V_{2}=0$ for the relations between the interlayer and intersite (intralayer)
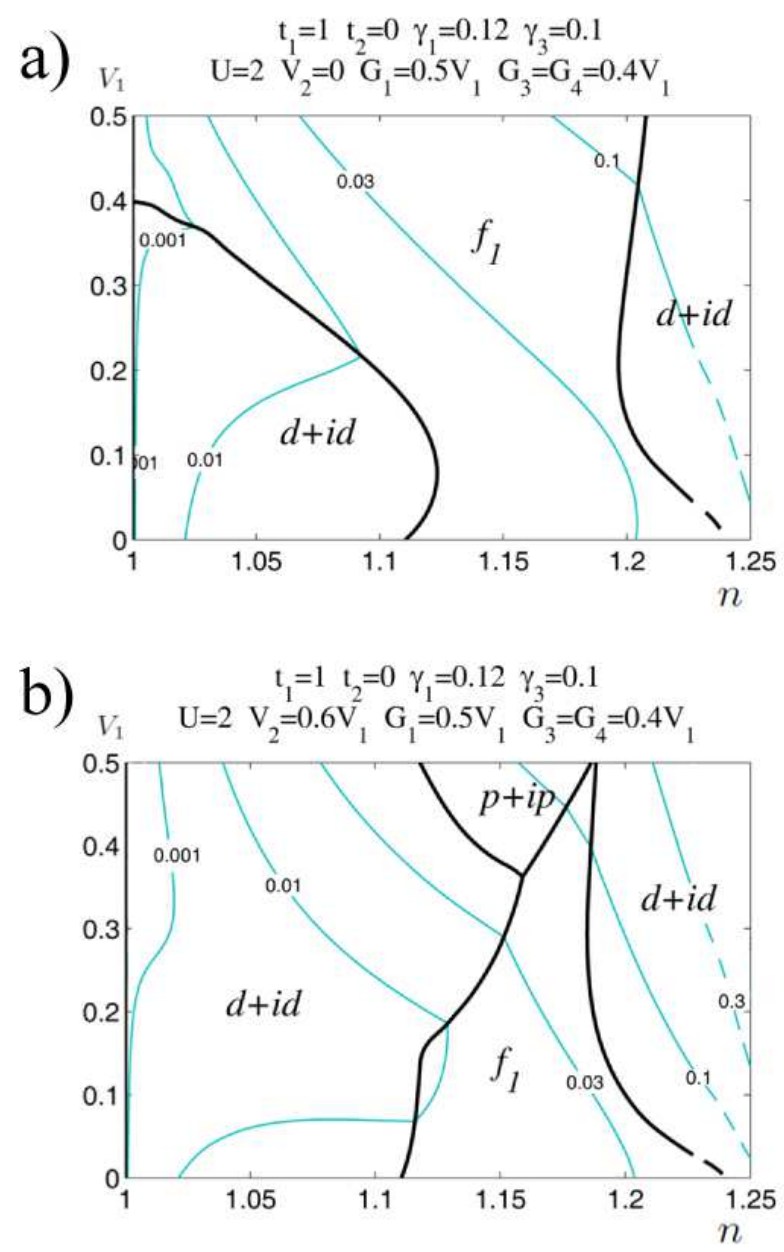

Fig. 17. The " $n-V_{1}$ " phase diagram of the superconducting state of the graphene bilayer at $t_{2}=0, \gamma_{1}=$ $0.12, \gamma_{3}=0.1, U=2, G_{1}=0.5 V_{1}, G_{3}=G_{4}=0.4 V_{1}$ at $V_{2}=0$ (a) and $V_{2}=0.6 V_{1}$ (b) (all the parameters are in the units of $\left.\left|t_{1}\right|\right)$. The thin lines are lines of constant $|\lambda|$ [231].

Coulomb interactions. In Fig. 17 $\mathrm{h}$, we chose the set of parameters $G_{1}=0.5 V_{1}, G_{3}=G_{4}=0.4 V_{1}$ in accordance with the hierarchy of the parameters specified by (77). The results of the calculations show that an increase in $G_{3}$ and $G_{4}$ separately leads to a suppression of the $d+i d$-wave pairing and to an expansion of the region of the $f$-wave pairing at low electron densities. In this case, a stronger suppression of the superconducting $d+i d$-wave phase can be achieved by an increase in the interlayer Coulomb interaction parameter $G_{4}$. Simultaneously, when we take the interactions $G_{3}$ and $G_{4}$ into account, as it is shown in Fig. 17a, we get not only an intensive suppression of the superconducting $d+i d$-wave pairing at low electron densities and realization of the superconductivity with the $f$-wave symmetry, but also an increase in the absolute values of the effective coupling constant $\lambda$. 
Figure 17b shows the phase diagram of a graphene bilayer calculated at the same parameters as in Fig. 17a, but with an account of the long-range Coulomb repulsion of electrons $V_{2}$. It can be seen from the comparison of Fig. 17b and Fig. 12b that the inclusion of $G_{3} \neq 0$ and $G_{4} \neq 0$ leads to a strong competition between the $d+i d-$ and $p+i p$-wave pairing and to a significant suppression of the latter in the region of the intermediate electron concentrations. In this case, in the preserved region of $p+i p$-wave pairing, the value of $\left|\lambda_{p+i p}\right|$ exceeds the $f$-wave coupling constant $\left|\lambda_{f}\right|$ insignificantly.

The inclusion of electron hoppings $t_{2}$ to the nextto-nearest carbon atoms does not affect qualitatively the competition between the superconducting phases shown in Fig. 17. This can be seen from Fig. 18, which shows the phase diagram of a graphene bilayer obtained with the parameter values $t_{2}=0.1, \gamma_{1}=$ $0.12, \gamma_{3}=0.1, U=2, G_{1}=0.5 V_{1}$ and $G_{3}=G_{4}=$ $0.4 V_{1}$. This behavior of the system is explained by the fact that the hopping $t_{2}>0$ or $t_{2}<0$ for the graphene bilayer, just as in the case of the monolayer, does not lead to an essential modification of the density of states in the regions of charge-carrier concentrations between the Dirac point and both $n_{V H}$ points (Fig. 19). However, it can be seen from Fig. 18 that an account for hoppings $t_{2}$ leads to an increase in the absolute values of the effective interaction and, consequently, to the higher critical temperatures of the superconducting transition in the idealized graphene bilayer.

Our calculations show that the Kohn-Luttinger

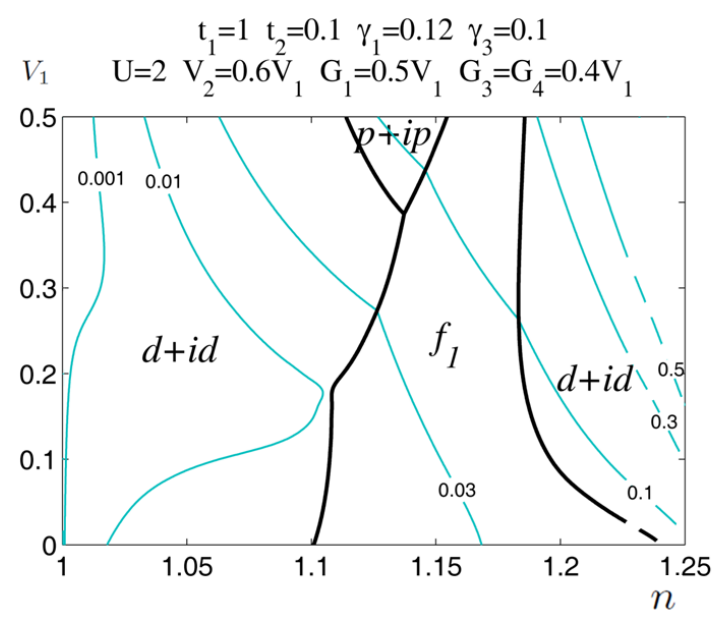

Fig. 18. The " $n-V_{1}$ " phase diagram of the superconducting state of a graphene bilayer at $t_{2}=0.1, \gamma_{1}=$ $0.12, \gamma_{3}=0.1, U=2, G_{1}=0.5 V_{1}, G_{3}=G_{4}=0.4 V_{1}$ (all the parameters are in the units of $\left|t_{1}\right|$ ). The thin lines are lines of constant $|\lambda|$ 231. mechanism can result in critical temperatures of the superconducting transition as high as $T_{c} \sim 20-40 \mathrm{~K}$ in the idealized graphene bilayer. In spite of these rather optimistic estimations, the superconductivity in real graphene, as it was stressed in Section $\$$, has not been discovered yet. Real graphene is only close to superconductivity.

There are several reasons why the results of the theoretical calculations presented in this review can be in disagreement with the experimental data. First of all, in our calculations, we did not take into account the influence of the van der Waals potential of the substrate 253 257. With an increase in the number of layers, the role of this potential should be weakened apparently. However, even in the case of a multilayer system, the van der Waals forces can worsen the conditions for the development of the Cooper instability.

Secondly, as we noted, there is no common opinion in the literature concerning the values of the parameters of the intraplane and interplane Coulomb interactions in the graphene bilayer. In this review, the values close to those obtained from ab initio calculations (performed in [226] for graphite) were used for the intraplane Coulomb interactions. The values of the interplane Coulomb interactions were taken such that they would satisfy the hierarchy of the parameters of the Born weak-coupling approximation.

Thirdly, in our calculations, a clean and structurally ideal graphene bilayer is considered, whereas a real material contains various impurities and structural imperfections, including grain boundaries and twinning planes. For conventional $s$-wave pairing, the singlet superconducting state is destroyed by magnetic impurities $99,258,260$, but for anomalous pairing with $f-, p+i p$-, and $d+i d$-wave symmetry even nonmagnetic impurities [98, and structural imperfections are known to contribute to the destruction of the superconducting state [250,261,311].

We also emphasize one additional possible reason for the discrepancy between the results of the theoretical calculations in graphene and experimental observations. In recent work 262, the role of quantum $(T=0)$ fluctuations in the graphene layers was investigated. These fluctuations were shown to initiate logarithmic corrections to the elasticity and bending moduli of the layers. In other words, according to 262, quantum fluctuations of the flexural vibrations of graphene layers can lead to a situation when the electrons move along strongly curved string-like trajectories rather than along the atomically smooth layers. This situation requires examination, although in this case superconductivity can not be excluded and can even be enhanced via the exchange by the 


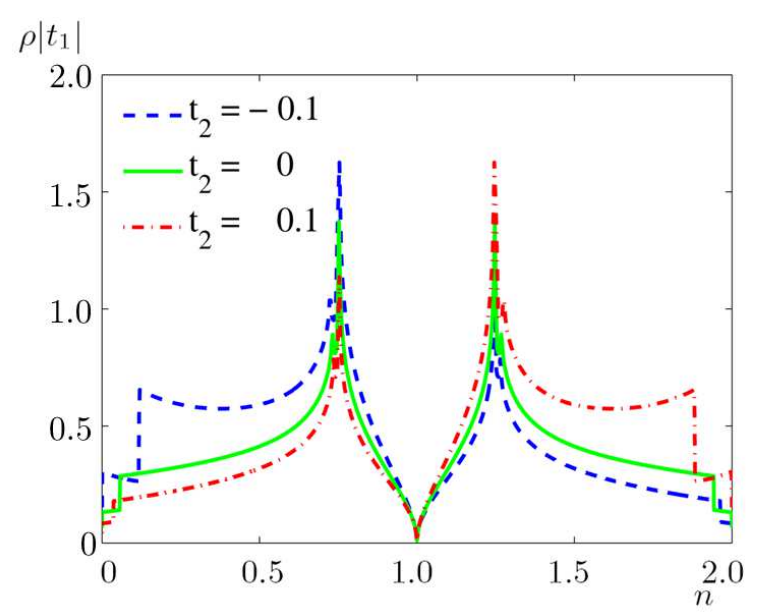

Fig. 19. Dependence of the density of states for the graphene bilayer per unit cell of one layer on the electron concentration for the set of parameters $t_{2}=0, \gamma_{1}=0.12\left|t_{1}\right|, \gamma_{3}=0.1\left|t_{1}\right|[231$.

quanta of the bending vibrations between the pairing electrons.

\section{New promising systems with anomalous pairing}

We can assume today that there is a number of systems in which anomalous superconducting pairing can be realized, and in particular, the pairing based on the Kohn-Luttinger mechanism and its generalizations. One such a system is strontium titanate $\mathrm{SrTiO}_{3}$. At room temperature, $\mathrm{SrTiO}_{3}$ has a cubic crystal structure, while at $T^{*}=105 \mathrm{~K}$, its structure becomes tetragonal as a result of a phase transition. The electron structure of $\mathrm{SrTiO}_{3}$ is characterized by the presence of an energy gap with a width of $3 \mathrm{eV}$, which separates the filled $2 p$ bands of oxygen from the empty $3 d$ bands of titanium [263,264].

It was shown in 265] that in a limited region of the momentum space near the center of the Brillouin zone, the band structure of the strontium titanate can be effectively described by $d_{x y}, d_{y z}$, or $d_{z x}$ Bloch waves, each having two directions with a strong dispersion ( $k_{x}$ and $k_{y}$ for the $d_{x y}$-wave orbital, etc.) and one direction with a weak dispersion, which is orthogonal to the first two directions [265]. As a result, three degenerate energy bands are formed, which with good accuracy can be approximated by parabolas, and the Fermi surface consists of three overlapping ellipsoids with the center at the center of the Brillouin zone, which are oriented along the axes $x, y$, and $z$ of the reciprocal cubic lattice.

Effectively, the strontium titanate is a semicon- ductor, which in the case of weak electronic doping demonstrates metallic properties with a relatively high mobility of charge carriers, quadratic temperature dependence of the electrical resistivity, and a strong temperature dependence of the infrared optical conductivity 265. At low temperatures, the material becomes superconducting [266], with the maximal critical temperature $T_{c}=1.2 \mathrm{~K}$ [50], although superconductivity is usually observed at lower temperatures, $T_{c} \leq 0.7 \mathrm{~K}$, and is characterized by a domelike $T_{c}(n)$ dependence [267, 268]. Superconducting pairing is also observed at $T_{c} \sim 0.2 \mathrm{~K}$ in the $2 \mathrm{D}$ electron gas, which is formed at the interface of the $\mathrm{SrTiO}_{3} / \mathrm{LaAlO}_{3}$ heterostructures [269, 270]. In this case, the superconducting transition temperature can be increased to $T_{c} \sim 0.3-0.4 \mathrm{~K}$ by the application of an electric field [271,272.

We note that at present the nature of superconductivity in strontium titanate and in related heterostructures remains unclear. Superconductivity in $\mathrm{SrTiO}_{3}$ was first investigated in Ref. 273. based on the mechanism of electron-electron attraction due to the exchange of phonons of the same and the different valleys. This study was stimulated by the earlier results of the band-structure calculations [274, which demonstrated the multivalley band structure of $\mathrm{SrTiO}_{3}$. Subsequently, the mechanism of multivalley superconductivity in strontium titanate was investigated in [275 277]. However, later on, when the data were accumulated indicating that $\mathrm{SrTiO}_{3}$ is a superconductor with three almost parabolic bands, other mechanisms of superconductivity, which are not connected with the multivalley structure were suggested (see, e.g., [278 280]).

In Ref. 281, in the framework of the phenomenological 2D model, were investigated manifestations of multiband superconductivity in thin films of $\mathrm{SrTiO}_{3}$ and at the interfaces of the $\mathrm{SrTiO}_{3} / \mathrm{LaAlO}_{3}$ heterostructures at various doping levels. The authors of [281] did not discuss the nature of the superconducting instability and limited themselves to an examination of $s$-wave pairing. In their model, the $d_{x z}$, $d_{y z}$, and $d_{x y}$-orbitals form two electron bands, as in a weakly doped compound, and only one of them intersects the Fermi level [281. With an increase in the doping level, the chemical potential intersects the second band, leading to a strong modification of the superconducting properties of the system. Theoretical results for the calculations of the dependences of $T_{c}$ and local density of states on the doping level 281. have been compared with the experimental data [268]. It was shown that the intraband (intraorbital) effective attraction in $\mathrm{SrTiO}_{3}$ prevails over the interband (interorbital) attraction, whereas in other multiband 
superconductors, such as in pnictides, no predominance of the intraband effective attraction over the interband one was observed.

Another systems in which the development of Cooper instability is possible via the Kohn-Luttinger mechanism is a family of the 'vertical' heterostructures, which consist of graphene layers separated by intercalating layers of boron nitride [282 285]. These structures demonstrate a number of interesting properties connected with the interaction of electrons from different layers. In these systems, the boron nitride (h-BN), like graphene, has a hexagonal structure (because of its color and the similarity of its structure to the structure of graphite, it is frequently called 'white graphite' [286]). At the same time, at the sites of the $A$ and $B$ sublattices, it contains atoms of boron and nitrogen. This causes the appearance of a wide energy gap (5.2-5.9 eV) in the electronic structure of h$\mathrm{BN}$ [287 291], which underlies the wide usage of h-BN as a high-quality dielectric in graphene devices [292]. Note that h-BN is chemically and thermally stable and is not characterized by the presence of broken bonds or surface traps for the charge carriers. That is why graphene structures based on h-BN substrates demonstrate a higher mobility of charge carriers 293, 294, smaller roughness, and twofold-lower fluctuations of the potential than the similar graphene structures on the substrates of $\mathrm{SiO}_{2}$ 295,296.

Graphene structures with h-BN can easily be modified. The concentration of charge carriers in the graphene layers, the spacings between these layers, and the nature of the substrate can be changed independently in a wide range of parameters. These changes can lead to a strong modification of electronelectron interaction in this family of heterostructures, which can open the possibility of realization of superconductivity at relatively small concentrations of carriers and in the absence of any specific properties of the density of states 297.

We note that the possibility of Cooper pairing due to electron-electron interaction was investigated in Ref. 297] in the model of a vertical heterostructure consisting of two graphene layers with the concentrations of carriers $n_{1}$ and $n_{2}$ and with three dielectric intercalating layers with different static dielectric constants $\epsilon_{1}, \epsilon_{2}$, and $\epsilon_{3}$. The possibility of the superconducting instabilities was analyzed in the framework of the Kohn-Luttinger mechanism and the most probable superconducting phase was described. In particular, it was shown that the superconducting state with odd momenta, at which the superconducting gaps have opposite signs in different Dirac cones, is the ground state of the system due to the intervalley scattering at high densities.
Note that in solids, the crystalline structure strictly determines the effective mass, the velocity of electrons, and the strength of their interactions. This constraint significantly limits the development and the verification of different theoretical and experimental methods of physics of strongly correlated electronic systems. Another, more flexible method for studying the different models of strongly correlated electrons is connected with the systems of ultracold atoms captured by a periodic potential obtained by the interference of three laser beams [298, 299].

In Ref. [300, an experimental scheme was suggested of simulation and observation of the Dirac fermions in a system of the ultracold atoms in a twodimensional hexagonal optical lattice. The authors of [300, 301] showed theoretically that it is possible to control the anisotropy of the optical lattice by changing the intensity of the trapping laser and realize both the regimes of massive and massless Dirac fermions, as well as to observe a phase transition between these two regimes. In fact, the authors of [300,301, predicted a topological semimetal-dielectric Lifshitz transition with the gap opening in the fermionic spectrum and with a change in the temperature behavior of the electronic heat capacity. It was noted in [300] that Bragg spectroscopy 302 and different methods of determining the atomic-density profile in magnetic or optical traps 303 305] can be used for the experimental detection of both gapped and gapless regimes and the phase transitions between them. The physical picture observed in this case, according to the authors of [300], must be very close to the picture of the ensemble of the Dirac fermions in the graphene monolayer.

Recently, the researchers of Eslinger's group [306] in Zurich experimentally realized the Dirac points with well controlled properties using the ultracold fermionic atoms of ${ }^{40} \mathrm{~K}$ in a hexagonal optical lattice. The presence of the Dirac points in the band structure was detected by the authors of 306 by the observation of a minimal gap in the Brillouin zone. The authors of 306] used the unique experimental technique of the optical tuning of the lattice potential for controlling the effective mass of the Dirac fermions. Moreover, the change in the lattice anisotropy allowed the authors of 306 to change the position of the Dirac points in the Brillouin zone. It turns out that if the anisotropy exceeds a certain critical value, then the two Dirac points merge and annihilate. This phenomenon generated a broad theoretical interest 301,307 309, but at the same time the difficulties concerning the possibility of its experimental observation in solids became obvious to the community 310 .

The experimental realization of the Dirac points 
in a system of the ultracold atoms in the hexagonal optical lattices 306] also opens the great prospects for the experimental and theoretical study of a broad class of the physical phenomena caused by the complex topology of the lattice, including the anomalous superconducting pairing and the appearance of the various chiral phases.

\section{Conclusions}

The Kohn-Luttinger mechanism and its generalizations (which assume the appearance of the anomalous pairing in the systems with a purely repulsive interaction) represent a universal pairing mechanism for many superconductive systems. We demonstrated the instability of the Fermi gas with repulsion towards the superconducting transition in a triplet $p$ wave state. The initial conclusion about the possibility of the Cooper instability for the Fermi-gas with a short-range (Hubbard) repulsion and a quadratic dispersion law was generalized for the electrons in the real crystalline solids in the tight-binding approximation. It turns out that the character of the energy spectrum of electrons (which is determined by the hopping parameters) greatly affects the symmetry of the superconducting order parameter and the phase diagram of superconductivity.

Nevertheless, in a fundamental sense, the conclusion about the development of the Cooper instability via the Kohn-Luttinger mechanism and its generalizations remains valid even in the presents of the lattice. Moreover, in many cases this mechanism can lead to a substantial increase in the superconducting transition temperature already at low density of charge carriers in particular in a spin-polarized case and in a twoband situation.

It has been demonstrated that the universality of the Kohn-Luttinger mechanism is preserved when we take into account the finite screening radius in electron systems with repulsion. In the framework of the Shubin-Vonsovsky model, we showed that the Coulomb repulsion of electrons located at different sites of the crystalline lattice could substantially modify the superconducting phase diagram and can lead to an increase of the critical temperature under appropriate conditions. In particular, at the electron concentrations close to the Van Hove singularity in the density of states the critical temperatures can reach the values of the order of $100 \mathrm{~K}$ (realistic for cuprates) at the moderate ratios of $U / W$ between the Hubbard repulsion $U$ and the bandwidth $W$.

We have also shown in this review that the KohnLuttinger mechanism of the superconducting pairing can be realized in the systems with a linear (Diraclike) dispersion relation. This possibility was demonstrated for an idealized graphene monolayer whose atoms form a hexagonal lattice. It was shown that in this system, the polarization effects also lead to the effective attraction of electrons in the Cooper channel. The results obtained for the graphene monolayer were generalized on the case of a graphene bilayer, which consists of two layers that interact by means of interlayer Coulomb repulsion. It was shown that the examination of the idealized two-layer system of graphene leads to an increase in the critical temperature of the superconducting transition in the framework of the Kohn-Luttinger mechanism.

Along with the analysis of the superconducting state, we also analyzed the structure of the normal state of the basic models with the Hubbard repulsion and found nontrivial corrections to the Fermi gas Galitskii-Bloom expansion caused by the presence of the upper Hubbard band in the lattice models or by the presence of a singularity due to the Landau $f$ function of quasiparticles interaction at low electron densities. However, these corrections do not destroy the Landau Fermi-liquid picture in three-dimensional and two-dimensional systems, and also preserve all the results concerning the realization of the superconducting pairing in them.

In the review, the significant attention has been paid to the description of the systems and materials important for the development of the microelectronics, such as the vertical heterostructures of graphene/boron-nitride/graphene, strontium titanate, and the related heterostructures. We have analyzed in details both an anomalous superconductivity and the possibility of fermionic superfluidity in $3 \mathrm{D}$ and $2 \mathrm{D}$ solutions of ${ }^{3} \mathrm{He}$ in ${ }^{4} \mathrm{He}$, and also in a system of ultracold ${ }^{6} \mathrm{Li}$ and ${ }^{40} \mathrm{~K}$ atoms in the magnetic traps and the optical lattices. Thus, we built a bridge connecting the interests of the solid-state and the low-temperature scientific communities.

To conclude, we emphasize once again the universal nature of the Kohn-Luttinger mechanism and its generalizations for the formation of the Cooper instability in repulsive Fermi systems and its importance for the realization of an anomalous superconducting and superfluid pairing with a nonzero value of the orbital angular momentum $(l \neq 0)$.

\section{Acknowledgments}

We are grateful to M A Baranov, A V Chubukov, D V Efremov, M V Feigel'man, V V Kabanov, K I Kugel', M S Marienko, N M Plakida, N V Prokof'ev, 
A Ya Tzalenchuk, and V V Val'kov for the fruitful discussions and constant attention to our work. The work was supported by the Russian Foundation for Basic Research (project nos. 14-02-00058 and 14-02-31237). M Yu K thanks the Program of Basic Research of the National Research University Higher School of Economics for support. The work of M M K was supported by grant of the President of the Russian Federation (SP-1361.2015.1) and the Dinasty Foundation.

\section{References}

1. Duan D et al. Sci. Rep. 46968 (2014)

2. Drozdov A P, Eremets M I, Troyan I A, Nature $\mathbf{5 2 5}$ 73 (2015)

3. Bardeen J, Cooper L, Schrieffer J Phys. Rev. 1081175 (1957)

4. Anderson P W Science 2351196 (1987)

5. Regal C A et al. Phys. Rev. Lett. 90053201 (2003)

6. Schunck C H et al. Phys. Rev. A 71045601 (2005)

7. Ott H R et al. Phys. Rev. Lett. 521915 (1984)

8. Kromer S et al. Phys. Rev. Lett. 814476 (1998)

9. Kuroki K J. Phys. Soc. Jpn. 75051013 (2006)

10. Maeno Y, Rice T M, Sigrist M Phys. Today 5442 (2001)

11. Rice T M, Sigrist M J. Phys.: Condens. Matter 7 L643 (1995)

12. Murase K et al. Surf. Sci. 170486 (1986)

13. Nagamatsu J et al. Nature 41063 (2001)

14. Kamihara Y et al. J. Am. Chem. Soc. 1303296 (2008)

15. Liu Y et al., arXiv:1503.08587

16. Vollhardt D, Woelfle P The superfluid phases of Helium 3 (London: Taylor and Francis, 1990)

17. Volovik G E Exotic properties of superfluid ${ }^{3} \mathrm{He}$ (Singapore: World Scientific, 1992)

18. Volovik G E The Universe in a Helium Droplet (Oxford: Clarendon Press, 2003)

19. Novoselov K S et al. Science 306666 (2004)

20. Kagan M Yu Phys. Usp. 3769 (1994)

21. Ong W et al. Phys. Rev. Lett. 114110403 (2015)

22. Ries M G et al. Phys. Rev. Lett. 114230401 (2015)

23. Lozovik Yu E, Merkulova S P, Sokolik A A Phys. Usp. 51727 (2008)

24. Kotov V N et al. Rev. Mod. Phys. 841067 (2012)

25. Wallace P R Phys. Rev. 71622 (1947)

26. Castro Neto A H et al. Rev. Mod. Phys. 81109 (2009)

27. Kohn W, Luttinger J M Phys. Rev. Lett. 15524 (1965)

28. Miyake K J. Phys.: Condens. Matter 19125201 (2007)

29. Gor'kov L P, Melik-Barchudarov T K Sov. Phys. JETP 131018 (1961)
30. Friedel J Adv. Phys. 3446 (1954)

31. Friedel J Nuovo Cimento Suppl. 2287 (1958)

32. Kagan M Yu et al. JETP 118995 (2014)

33. Kagan M Yu et al. Solid State Commun. 18861 (2014)

34. Lindhard J K. Dan. Vidensk. Selsk. Mat. Fys. Medd. 288 (1954)

35. Ashcroft N, Mermin N Solid State Physics (New York: Rinehart and Winston, 1976, Vol. 1)

36. Migdal A B Sov. Phys. JETP 7996 (1958)

37. Kohn W Phys. Rev. Lett. 2393 (1959)

38. Fay D, Layzer A Phys. Rev. Lett. 20187 (1968)

39. Kagan M Yu, Chubukov A V JETP Lett. 47614 (1988)

40. Baranov M A, Chubukov A V, Kagan M Yu Int. J. Mod. Phys. B 62471 (1992)

41. Baranov M A, Kagan M Yu, Kagan Yu JETP Lett. 64301 (1996)

42. Galitskii V M Sov. Phys. JETP 7104 (1958)

43. Kagan M Yu, Chubukov A V JETP Lett. 50517 (1989)

44. Chubukov A V Phys. Rev. B 481097 (1993)

45. Efremov D V et al. Physica B 284-288 216 (2000)

46. Bloom P Phys. Rev. B 12125 (1975)

47. Afanas'ev A M, Kagan Yu Sov. Phys. JETP 161030 (1962)

48. Efremov D V et al. JETP 90861 (2000)

49. Oh G-H et al. J. Low Temp. Phys. 95525 (1994)

50. Bednorz J G, Müller K A Z. Phys. B 64189 (1986)

51. Hubbard J C Proc. R. Soc. London A 276238 (1963)

52. Izyumov Yu A, Katsnelson M I, Skryabin Yu N Magnetism of Itinerant Electrons (Moscow: Fizmatlit, 1994)

53. Izyumov Yu A Phys. Usp. 38385 (1995)

54. Georges A et al Rev. Mod. Phys. 6813 (1996)

55. Tasaki H J. Phys.: Condens. Matter. 684353 (1998)

56. Ovchinnikov S G, Val'kov V V Hubbard Operators in the Theory of Strongly Correlated Electrons (London: Imperial College Press, 2004)

57. Baranov M A, Kagan M Yu Z. Phys. B 86237 (1992)

58. Baranov M A, Kagan M Yu Sov. Phys. JETP 72689 (1991)

59. Scalapino D J, Loh Jr E, Hirsch J E Phys. Rev. B 34 8190 (1986)

60. Scalapino D J, Loh Jr E, Hirsch J E Phys. Rev. B 35 6694 (1987)

61. Kozlov A N Sverkhprovodimost Fiz. Khim. Tekh. 264 (1989)

62. Dzyaloshinskii I E, Krichever I M, Chronek J Sov. Phys. JETP 671492 (1988)

63. Hlubina R Phys. Rev. B 599600 (1999)

64. Zanchi D, Schulz H J Phys. Rev. B 549509 (1996)

65. Dzyaloshinskii I E, Yakovenko V M Sov. Phys. JETP 67844 (1988) 
66. Zheleznyak A T, Yakovenko V M, Dzyaloshinskii I E Phys. Rev. B 553200 (1997)

67. Raghu S, Kivelson S A, Scalapino D J Phys. Rev. B 81224505 (2010)

68. Kagan M Yu Phys. Lett. A 152303 (1991)

69. Kagan M Yu, Val'kov V V JETP 140179 (2011).

70. Kagan M Yu, Val'kov V V, in A Lifetime in Magnetism and Superconductivity: A Tribute to Professor David Schoenberg (Cambridge: Cambridge Scientific Publishers, 2011)

71. Falicov L M, Kimball J C Phys. Rev. Lett. 22997 (1969)

72. Kagan Yu, Prokof'ev N V Sov. Phys. JETP 631276 (1986)

73. Kagan Yu, Prokof'ev N V Sov. Phys. JETP 66211 (1987)

74. Suhl H, Matthias T B, Walker L R Phys. Rev. Lett. 3552 (1959)

75. Geilikman B T Sov. Phys. JETP 21796 (1965)

76. Geilikman B T Sov. Phys. Usp. 9142 (1966)

77. Geilikman B T Sov. Phys. Usp. 1617 (1973)

78. Baranov M A, Kagan M Yu JETP 75165 (1992)

79. Baranov M A, Efremov D V, Kagan M Yu Physica C 21875 (1993)

80. Frossati G et al. Czeck. J. Phys. 440909 (1990)

81. Wiegers S A J et al. Physica B 165-166 733 (1990)

82. Bedell K S, Sanchez-Castro C Phys. Rev. Lett. 57854 (1986)

83. Frossati G et al. Phys. Rev. Lett. 571032 (1986)

84. Dy K S, Pethick C J Phys. Rev. 185373 (1969)

85. Bashkin E P, Meyerovich A E Adv. Phys. 301 (1981)

86. Østgaard E, Bashkin E Physica B 178134 (1992)

87. Andreev A F Sov. Phys. JETP 23939 (1966)

88. Zinov'eva K N, Boldarev S T Sov. Phys. JETP 29 585 (1969)

89. Alikacem N, Sprague D T, Hallock R B Phys. Rev. Lett. 672501 (1991)

90. Higley R H, Sprague D T, Hallock R B Phys. Rev. Lett. 632570 (1989)

91. Saunders J, Lusher C P, Cowan B P Phys. Rev. Lett. 642523 (1990)

92. Lusher C P, Cowan B P, Saunders J Phys. Rev. Lett. 672497 (1991)

93. Miyake K Prog. Theor. Phys. 691794 (1983)

94. Randeria M, Duan J-M, Shieh L-Y Phys. Rev. Lett. 62981 (1989)

95. Schmitt-Rink S, Varma C M, Ruckenstein A E Phys. Rev. Lett. 63445 (1989)

96. Kagan M Yu Modern Trends in Superconductivity and Superfluidity. Lecture Notes in Physics (Springer Netherlands, Dordrecht 2014, Vol. 874)

97. Stoof H T C et al. Phys. Rev. Lett. 7610 (1996)

98. Larkin A I Sov. Phys. JETP 31784 (1970)

99. Abrikosov A A, Gor'kov L P Sov. Phys. JETP 12337 (1961)
100. Anderson P W Phys. Rev. Lett. 652306 (1990)

101. Varma C M et al., Phys. Rev. Lett. 631996 (1989)

102. Anderson P W Phys. Rev. Lett. 663226 (1991)

103. Engelbrecht J R, Randeria M Phys. Rev. Lett. 65 1032 (1990)

104. Fukuyama H, Hasegawa Y, Narikiyo O J. Phys. Soc. Jpn. 602013 (1991)

105. Fabrizio M, Tossati E, Parola A, Doctoral Thesis (M. Fabrizio) (1992)

106. Prokofiev N V, In review article: Stamp P J. de Phys. (Paris) 3625 (1993)

107. Baranov M A, Kagan M Yu, Mar'enko M S JETP Lett. 58709 (1993)

108. Kagan M Yu, Val'kov V V, Woelfle P Low Temp. Phys. 371046 (2011)

109. Kanamori J Progr. Theor. Phys. 30275 (1963)

110. Alexandrov A S, Kabanov V V Phys. Rev. Lett. 106 136403 (2011)

111. Shubin S P, Vonsovsky S V Proc. Roy. Soc. A 145 159 (1934)

112. Shubin S P, Vonsovsky S V Sow. Phys. 7292 (1935)

113. Shubin S P, Vonsovsky S V Sow. Phys. 10348 (1936)

114. Vonsovsky S V, Katsnelson M I J. Phys. C: Solid State Phys. 122043 (1979)

115. Vonsovsky S V, Katsnelson M I J. Phys. C: Solid State Phys. 122055 (1979)

116. Zaitsev R O Sov. Phys. JETP 51671 (1980)

117. Zaitsev R O, Ivanov V A, Mikhailova Yu V Fiz. Met. Metalloved. 651032 (1988)

118. Zaitsev R O, Ivanov V A, Mikhailova Yu V Fiz. Met. Metalloved. 651108 (1989)

119. Val'kov V V, Korovushkin M M JETP 112108 (2011)

120. Kagan M Yu et al. JETP Lett. 93819 (2011)

121. Landau L D, Lifshitz E M Quantum Mechanics: Non-Relativistic Theory (Oxford: ButterworthHeinemann, 1991)

122. Raghu S et al. Phys. Rev. B 85024516 (2012)

123. Kagan M Yu et al. JETP Lett. 97226 (2013)

124. Kagan M Yu et al. JETP 117728 (2013)

125. Deng Y et al., Europhys. Lett. 11057001 (2015)

126. Okazaki K et al. Science 3371314 (2012)

127. Izyumov Yu A Sov. Phys. Usp. 34935 (1991)

128. Brenig W Phys. Rep. 251153 (1995)

129. Izyumov Yu A Phys. Usp. 40445 (1997)

130. Plakida N M Condens. Mat. Phys. 4707 (2002)

131. Bulaevskii L N, Nagaev E L, Khomskii D I Sov. Phys. JETP 27836 (1968)

132. Chao K A, Spałek J, Oleś A M J. Phys. C 10 L271 (1977)

133. Hybertsen M S, Schluter M, Christensen N E Phys. Rev. B 399028 (1989)

134. Unger P, Fulde P Phys. Rev. B 478947 (1993)

135. Emery V J Phys. Rev. Lett. 582794 (1987) 
136. Varma C M, Schmitt-Rink S, Abrahams E Solid State Commun. 62681 (1987)

137. Kagan M Yu, Rice T M J. Phys.: Condens. Matter 63771 (1994)

138. Plakida N M JETP Lett. 7436 (2001)

139. Plakida N M et al. JETP 97331 (2003)

140. Hubbard J C Proc. R. Soc. London A 285542 (1965)

141. Zhang F C, Rice T M Phys. Rev. B 373759 (1988)

142. Zaitsev R O, Ivanov V A Sov. Phys. Solid State 29 475 (1987)

143. Zaitsev R O JETP 98780 (2004)

144. Laughlin R B Phys. Rev. Lett. 602677 (1988)

145. Fetter A L, Hanna C B, Laughlin R B Phys. Rev. B 399679 (1989)

146. Kagan M Yu et al. Phys. Rev. A 70023607 (2004)

147. Kagan M Yu et al. Phys. Usp. 491079 (2006)

148. Brinkman W F, Rice T M Phys. Rev. B 21324 (1970)

149. Sachdev S Phys. Stat. Sol. 247537 (2010)

150. Lee J, Strack P, Sachdev S Phys. Rev. B 87045104 (2013)

151. Castellani C, Di Castro C, Grilli M Phys. Rev. Lett. 754650 (1995)

152. Zaitsev R O Sov. Phys. JETP 41100 (1975)

153. Zaitsev R O Sov. Phys. JETP 43574 (1976)

154. Eremin M, Eremin I, Varlamov S Phys. Rev. B 64 214512 (2001)

155. Eremin M V, Shigapov I M, Eremin I M Eur. Phys. J. B 85131 (2012)

156. Plakida N M, Oudovenko V S Eur. Phys. J. B 86 115 (2013)

157. Plakida N M, Oudovenko V S JETP 119554 (2014)

158. Hirsch J E Phys. Lett. A 136153 (1989)

159. Yushankhai V Yu, Vujicic G M, Zakula R B Phys. Lett. A 151254 (1990)

160. Val'kov V V et al. JETP Lett. 75378 (2002)

161. Val'kov V V, Dzebisashvili D M JETP 100608 (2005)

162. Korshunov M M, Ovchinnikov S G, Sherman A V JETP Lett. 8039 (2004)

163. Val'kov V V, Golovnya A A JETP 107996 (2008)

164. Val'kov V V, Korovushkin M M, Barabanov A F JETP Lett. 88370 (2008)

165. Val'kov V V et al. Phys. Solid State 531997 (2011)

166. Bermudez A, Porras D New J. Phys. 17103021 (2015)

167. Marienko M S, Sau J D, Tewari S, arXiv:1202.5784

168. Novoselov K S et al. Nature (London) 438197 (2005)

169. Tan Y-W et al. Phys. Rev. Lett. 99246803 (2007)

170. Morozov S V et al. Phys. Rev. Lett. 100016602 (2008)

171. Bolotin K I et al. Solid State Commun. 146351 (2008)

172. Garcia N et al. Phys. Rev. B 78035413 (2008)
173. Geim A K, Katsnelson M I, Novoselov K S Nature Phys. 2620 (2006)

174. Young A F, Kim P Nature Phys. 5222 (2009)

175. Katsnelson M I Eur. Phys. J. B 51157 (2006)

176. Rusin T M, Zawadzki W Phys. Rev. B 80045416 (2009)

177. Nair P R et al. Science 3201308 (2008)

178. Muñoz W A, Covaci L, Peeters F M Phys. Rev. B 86184505 (2012)

179. Heersche H B et al. Nature (London) 44656 (2007)

180. Josephson B D Phys. Lett. 1251 (1962)

181. Shailos A et al. Europhys. Lett. 7957008 (2007)

182. Titov M, Beenakker C W J Phys. Rev. B $74041401 \mathrm{R}$ (2006)

183. Du X, Skachko I, Andrei E Y Phys. Rev. B 77184507 (2008)

184. Ojeda-Aristizabal C et al. Phys. Rev. B 79165436 (2009)

185. Tomori H et al. Physica C 4701492 (2010)

186. Peres N M R, Guinea F, Castro Neto A H Phys. Rev. B 72174406 (2005)

187. Esquinazi P Physics 5050007 (2013)

188. Marino E C, Nunes L H C M Nucl. Phys. B 741404 (2006)

189. González J, Guinea F, Vozmediano M A H Phys. Rev. B 63134421 (2001)

190. Zhao E, Paramekanti A Phys. Rev. Lett. 97230404 (2006)

191. Uchoa B, Castro Neto A H Phys. Rev. Lett. 98 146801 (2007)

192. Kopnin N B, Sonin E B Phys. Rev. Lett. 100246808 (2008)

193. Basko D M, Aleiner I L Phys. Rev. B 77 041409(R) (2008)

194. Lozovik Yu E, Ogarkov S L, Sokolik A A Phil. Trans. R. Soc. A 3685417 (2010)

195. Einenkel M, Efetov K B Phys. Rev. B 84214508 (2011)

196. Classen L, Scherer M M, Honerkamp C Phys. Rev. B 90035122 (2014)

197. Black-Schaffer A M, Doniach S Phys. Rev. B 75 134512 (2007)

198. Black-Schaffer A M, Wu W, Le Hur K Phys. Rev. B 90054521 (2014)

199. Black-Schaffer A M, Honerkamp C J. Phys.: Condens. Matter 26423201 (2014)

200. Krishana K et al. Science 27783 (1997)

201. Elhalel K et al. Phys. Rev. Lett. 98137002 (2007)

202. Balatsky A V Phys. Rev. Lett. 801972 (1998)

203. Volovik G E Phys. Lett. A 128277 (1988)

204. Biswas P K et al. Phys. Rev. B 87 180503(R) (2013)

205. Nishikubo Y, Kudo K, Nohara M J. Phys. Soc. Jpn. 80055002 (2011)

206. Honerkamp C Phys. Rev. Lett. 100146404 (2008) 
207. Daul S, Scalapino D J, White S R Phys. Rev. Lett. 844188 (2000)

208. Ma T et al. Phys. Rev. B $84121410(\mathrm{R})$ (2011)

209. Chen K S et al.Phys. Rev. B 88 041103(R) (2013)

210. González J Phys. Rev. B 78205431 (2008)

211. Markiewicz R S J. Phys. Chem. Solids 581179 (1997)

212. Valenzuela B, Vozmediano M A H New J. Phys. 10 113009 (2008)

213. McChesney J L et al. Phys. Rev. Lett. 104136803 (2010)

214. Gofron K et al. Phys. Rev. Lett. 733302 (1994)

215. Van Hove L Phys. Rev. 891189 (1953)

216. Nandkishore R, Levitov L S, Chubukov A V Nature Phys. 8158 (2012)

217. Nandkishore R, Chern G-W, Chubukov A V Phys. Rev. Lett. 108227204 (2012)

218. Dzyaloshinskii I E Sov. Phys. JETP 66848 (1987)

219. Schulz H J Europhys. Lett. 4609 (1987)

220. Shankar R Rev. Mod. Phys. 66129 (1994)

221. Makogon D et al. Phys. Rev. B 84125404 (2011)

222. Li T Europhys. Lett. 9737001 (2012)

223. Wang W S et al. Phys. Rev. B 85035414 (2012)

224. Nandkishore R, Chubukov A V Phys. Rev. B 86 115426 (2012)

225. Kiesel M L et al. Phys. Rev. B $86020507(\mathrm{R})$ (2012)

226. Wehling T O et al. Phys. Rev. Lett. 106236805 (2011)

227. Levin A A Solid State Quantum Chemistry (New York: McGraw-Hill, 1977)

228. Reich S et al. Phys. Rev. B 66035412 (2002)

229. Perfetto E et al. Phys. Rev. B 76233408 (2007)

230. Nandkishore R, Thomale R, Chubukov A V Phys. Rev. B 89144501 (2014)

231. Kagan M Yu, Mitskan V A, Korovushkin M M, Eur. Phys. J. B 88157 (2015)

232. Zaitsev R O JETP Lett. 94206 (2011)

233. Zaitsev R O JETP Lett. 95380 (2012)

234. Guinea F, Castro Neto A H, Peres N M R Phys. Rev. B 73245426 (2006)

235. Kagan M Yu, Mitskan V A, Korovushkin M M JETP 1191140 (2014)

236. McCann E, Fal'ko V I Phys. Rev. Lett. 96086805 (2006)

237. Nilsson J et al. Phys. Rev. B 73214418 (2006)

238. Partoens B, Peeters F M Phys. Rev. B $\mathbf{7 4} 075404$ (2006)

239. McCann E, Abergel D S L, Fal'ko V I Eur. Phys. J. Special Topics 14891 (2007)

240. McCann E, Koshino M Rep. Prog. Phys. 76056503 (2013)

241. Nunes L H C M, Mota A L, Marino E C Solid State Commun. 1522082 (2012)

242. Vučičević J, Goerbig M O, Milovanović M V Phys. Rev. B 86214505 (2012)
243. Milovanović M V, Predin S Phys. Rev. B 86195113 (2012)

244. Hosseini M V, Zareyan M Phys. Rev. Lett. 108 147001 (2012)

245. Hosseini M V, Zareyan M Phys. Rev. B 86214503 (2012)

246. Alford M, Rajagopal K, Wilczek F Nucl. Phys. B 537443 (1999)

247. Sedrakian A, Lombardo U Phys. Rev. Lett. 84602 (2000)

248. Hwang E H, Das Sarma S Phys. Rev. Lett. 101 $156802(2012)$

249. González J Phys. Rev. B 88125434 (2013)

250. Murray J M, Vafek O Phys. Rev. B 89205119 (2014)

251. Dresselhaus M S, Dresselhaus G Adv. Phys. 511 (2002)

252. Brandt N B, Chudinov S M, Ponomarev Y G, in Modern Problems in Condensed Matter Sciences (eds V M Agranovich, A A Maradudin) (Amsterdam: NorthHolland, Amsterdam, 1988, Vol 20.1)

253. Girifalco L A, Hodak M Phys. Rev. B 65125404 (2002)

254. Hasegawa M, Nishidate K Phys. Rev. B 70205431 (2004)

255. Hasegawa M, Nishidate K, Iyetomi H Phys. Rev. B 76115424 (2007)

256. Boström M, Sernelius B E Phys. Rev. A 85012508 (2012)

257. Klimchitskaya G L, Mostepanenko V M Phys. Rev. B 87075439 (2013)

258. Anderson P W Phys. Rev. Lett. 3325 (1959)

259. Tsuneto T Prog. Theor. Phys. 28857 (1962)

260. Markowitz D, Kadanoff L P Phys. Rev. 131563 (1963)

261. Löthman T, Black-Schaffer A M Phys. Rev. B 90 224504 (2014)

262. Kats E I, Lebedev V V Phys. Rev. B 89125433 (2014)

263. Cardona M Phys. Rev. 140 A651 (1965)

264. Shanthi N, Sarma D D Phys. Rev. B 572153 (1998)

265. van der Marel D, van Mechelen J L M, Mazin I I Phys. Rev. B 84205111 (2011)

266. Schooley J F, Hosler W R, Cohen M L Phys. Rev. Lett. 12474 (1964)

267. Koonce C S et al. Phys. Rev. 163380 (1967)

268. Binning G et al. Phys. Rev. Lett. 451352 (1980)

269. Reyren N et al. Science 3171196 (2007)

270. Heber J Nature (London) 45928 (2009)

271. Caviglia A D et al. Nature (London) 456624 (2008)

272. Bell C et al. Phys. Rev. Lett. 103226802 (2009)

273. Cohen M L Phys. Rev. 134 A511 (1964)

274. Kahn A H, Leyendecker A J Phys. Rev. 135 A1321 (1964)

275. Eagles D M Phys. Rev. 164489 (1967)

276. Eagles D M Phys. Rev. 186456 (1969) 
277. Koonce C S, Cohen M L Phys. Rev. 177707 (1969)

278. Appel J Phys. Rev. 180508 (1969)

279. Zinamon Z Philos. Mag. 21347 (1970)

280. Jarlborg T Phys. Rev. B 619887 (2000)

281. Fernandes R M et al. Phys. Rev. B 87014510 (2013)

282. Ponomarenko L A et al. Nat. Phys. 7958 (2011)

283. Brittnell L et al. Science 335947 (2012)

284. Brittnell L et al. Nano Lett. 121707 (2012)

285. Gorbachev R V et al. Nat. Phys. 8896 (2012)

286. Sakai Y, Koretsune T, Saito S Phys. Rev. B 83 205434 (2011)

287. Hoffman D M, Doll G L, Eklund P C Phys. Rev. B 306051 (1984)

288. Blase X et al. Phys. Rev. B 516868 (1995)

289. Watanabe K, Taniguchi T, Kanda H Nat. Mater. 3 404 (2004)

290. Kubota Y et al. Science 317932 (2007)

291. Lee G-H et al. Appl. Phys. Lett. 99243114 (2011)

292. Dean C R et al. Nat. Nanotechnol. 5722 (2010)

293. Mayorov A S et al. Nano Lett. 112396 (2011)

294. Gannett W et al. Appl. Phys. Lett. 98242105 (2011)

295. Xue J et al. Nat. Mater. 10282 (2011)

296. Decker R et al. Nano Lett. 112291 (2011)

297. Guinea F, Uchoa B Phys. Rev. B 86134521 (2012)

298. Lewenstein M et al. Adv. Phys. 56243 (2007)

299. Bloch I, Dalibard J, Zwerger W Rev. Mod. Phys. 80 885 (2008)

300. Zhu S-L, Wang B, Duan L-M Phys. Rev. Lett. 98 260402 (2007)

301. Wunsch B, Guinea F, Sols F New J. Phys. 10103027 (2008)

302. Stamper-Kurn D M et al. Phys. Rev. Lett. 832876 (1999)

303. Anglin J R, Ketterle W Nature (London) 416211 (2002)

304. Zwierlein M W et al. Science 311492 (2006)

305. Shin Y et al. Phys. Rev. Lett. 97030401 (2006)

306. Tarruell L et al. Nature Lett. 483302 (2012)

307. Hasegawa Y et al. Phys. Rev. B 74033413 (2006)

308. Montambaux G et al. Phys. Rev. B 80153412 (2009)

309. Lee K L et al. Phys. Rev. A 80043411 (2009)

310. Pereira V M, Castro Neto A H, Peres N M R Phys. Rev. B 80045401 (2009)

311. Posazhennikova A I, Sadovskii M V JETP Lett. 63 358 (1996) 